\title{
Science and Technology of Future Light Sources
}

\section{A White Paper}

Report prepared by scientists from ANL, BNL, LBNL and SLAC. The coordinating team consisted of Uwe Bergmann, John Corlett, Steve Dierker, Roger Falcone, John Galayda, Murray Gibson, Jerry Hastings, Bob Hettel, John Hill, Zahid Hussain, Chi-Chang Kao, Janos Kirz, Gabrielle Long, Bill McCurdy, Tor Raubenheimer, Fernando Sannibale, John Seeman, Z.-X. Shen, Gopal Shenoy, Bob Schoenlein, Qun Shen, Brian Stephenson, Joachim Stöhr, and Alexander Zholents. Other contributors are listed at the end of the document.

Argonne National Laboratory

Brookhaven National Laboratory

Lawrence Berkeley National Laboratory

SLAC National Accelerator Laboratory 
Argonne National Laboratory

9700 Cass Avenue

Argonne, IL 60439

Brookhaven National Laboratory

P.O. Box 5000

Upton, NY 11973-5000

Lawrence Berkeley National Laboratory

University of California

Berkeley, CA 94720

SLAC National Accelerator Laboratory

2575 Sand Hill Road

Menlo Park, CA 94025

\section{Editors:}

Arthur L. Robinson (LBNL) and Brad Plummer (SLAC)

\section{Illustrations and layout by:}

Terry Anderson, Gregory Stewart, Sharon West, InfoMedia Solutions (SLAC)

\section{DISCLAIMER}

This document was prepared as an account of work sponsored by the United States Government. While this document is believed to contain correct information, neither the United States Government nor any agency thereof, nor any of their employees, nor any of their contractors, subcontractors, or their employees, makes any warranty, express or implied, or assumes any legal liability or responsibility for the accuracy, completeness, or any third party's use or the results of such use of any information, apparatus, product, or process disclosed, or represents that its use would not infringe privately owned rights. Reference herein to any specific commercial product, process, or service by its trade name, trademark, manufacturer, or otherwise, does not necessarily constitute or imply its endorsement, recommendation, or favoring by the United States Government or any agency thereof or its contractors or subcontractors. The views and opinions of authors expressed herein do not necessarily state or reflect those of the United States Government or any agency thereof or its contractors or subcontractors.

This work was supported by the Director, Office of Science, Office of Basic Energy Sciences, of the U.S. Department of Energy under Contracts No. DE-AC02-06CH11357 (ANL), DE-AC02-98CH10886 (BNL), DEAC02-05CH11231 (LBNL) and DE-AC02-76SF00515 (SLAC). 


\section{Contents}

Contents.

Preface...

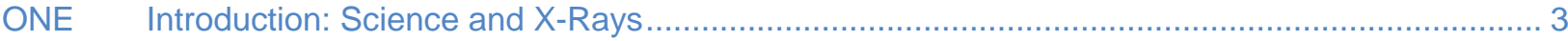

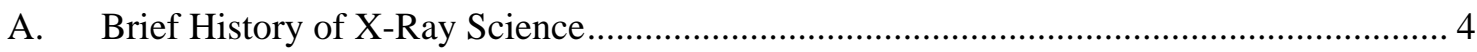

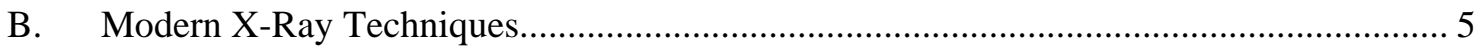

TWO Overview of Scientific Drivers and Required Enhanced X-Ray Capabilities.............................9 9

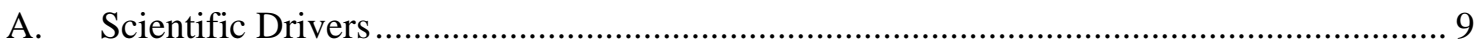

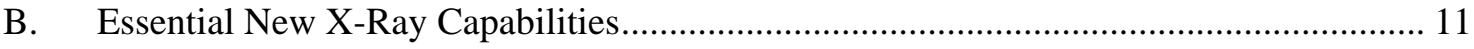

1 X-Ray Time Structure-Complete Control of Longitudinal Phase Space.................. 11

2 Full Transverse Coherence ................................................................................. 12

3 High Average Flux and Brightness ................................................................... 12

4 Tunability, Polarization Control, and Extended Photon Energies.............................. 12

THREE Examples of Scientific Drivers for Future X-Ray Sources ............................................. 15

A. Understanding and Controlling Electronic, Atomic, and Molecular Dynamics on Their

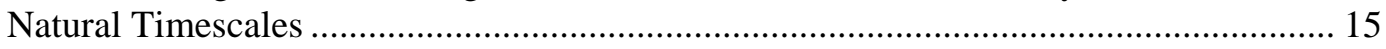

1 Attosecond Manipulation and Control of the Correlated Motion of Electrons .......... 16

2 Understanding Chemical Dynamics When the Timescales of Nuclear and Electronic Motion Can Coincide.....

3 Detecting the Correlated Motion of Electrons with a New Kind of X-Ray Spectroscopy

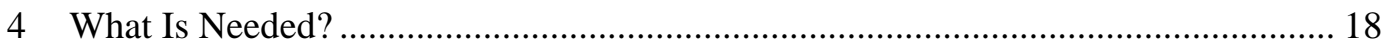

B. Chemical Reactivity: From Nature’s Catalysts to Controlled Reactions......................... 19

1 Understanding Light-Induced Catalysis_Learning from Nature............................... 19

2 Real-Time Catalysis and Surface Chemistry ........................................................... 20

3 Heterogeneous Chemistry of Aerosols-Geoengineering and Health....................... 22

4. Far from Equilibrium Chemical Processes in a Functional Solar Cell........................ 23

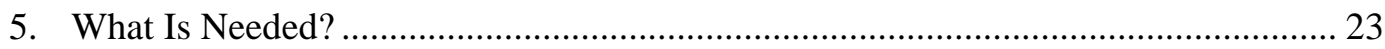

C. Understanding Complex Materials ........................................................................... 24

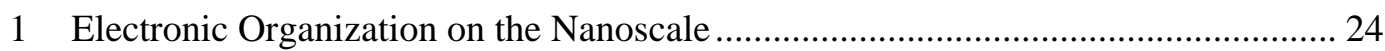

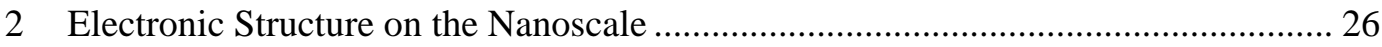

3 Electron Dynamics of Correlated Materials........................................................... 27

4 Nanoscale Dynamics: Equilibrium Fluctuations ........................................................ 29

5 Nanoscale Dynamics: Driven Phenomena ............................................................... 31

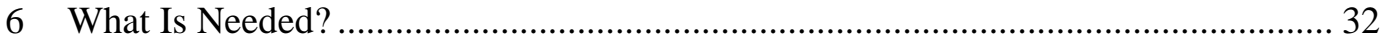

D. Novel Materials by Design and Materials Behavior under Extreme Conditions.............. 33

1 Tailoring Materials with Emergent Properties ............................................................ 34

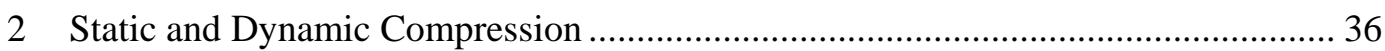

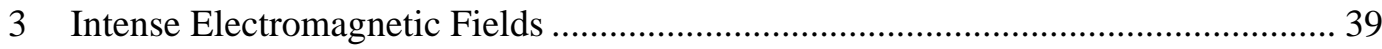




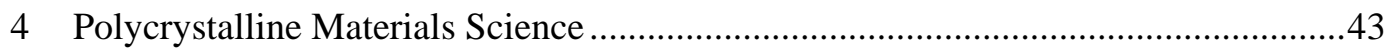

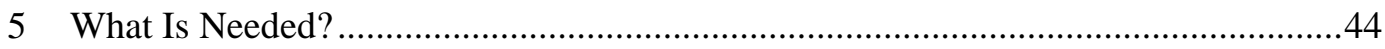

E. Life Science and Soft Condensed Matter .....................................................................44

1 Crystallography on Very Small Crystals ...............................................................45

2 In-situ Studies of Conformational Changes ..........................................................46

3 Nanoscale Structure Dynamics ...............................................................................

4 Structural Biology at the Cellular Level ..............................................................48

5 Trace Element Mapping.....................................................................................49

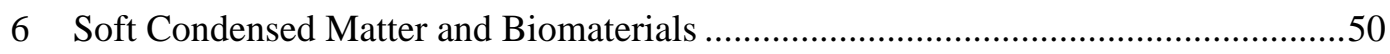

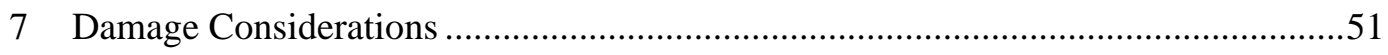

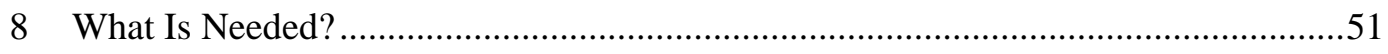

FOUR X-Ray Source Types, Capabilities, and Their Trade-Offs …...............................................53

A. Type of X-ray Sources with Enhanced Capabilities...........................................................53

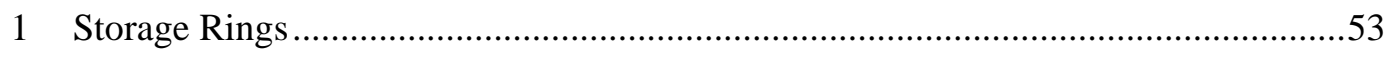

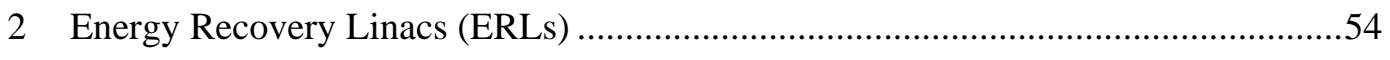

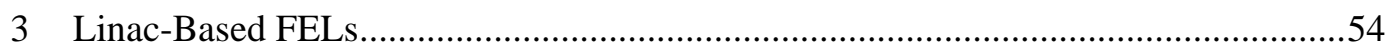

4 Graphical Representation of Source Capabilities .....................................................55

B. Discussion of Relative Capabilities and Trade-Offs ..................................................60

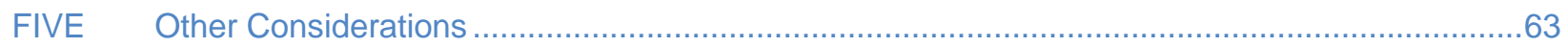

A. U.S. X-ray Sources in the International Environment.................................................63

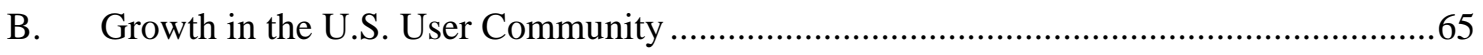

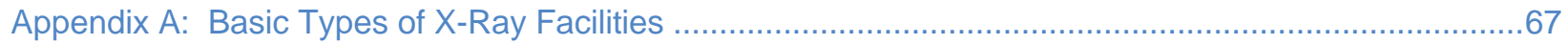

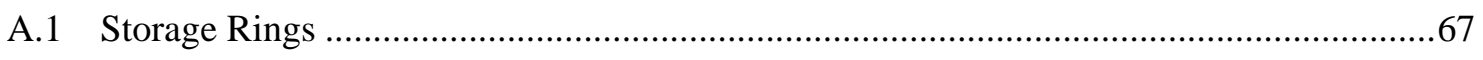

A.2. Energy Recovery Linacs (ERLs) ……...................................................................68

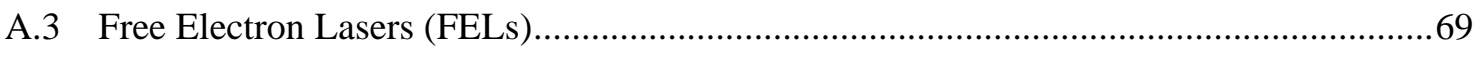

A.4 High-Harmonic Generation (HHG) .......................................................................... 71

A.5 Advanced Accelerator Concepts ........................................................................... 72

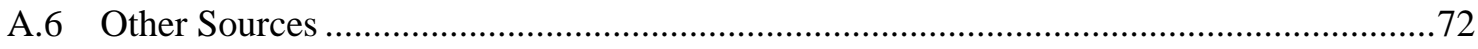

Appendix B: Research and Development Requirements for Sources, Optics, and Detectors..................73

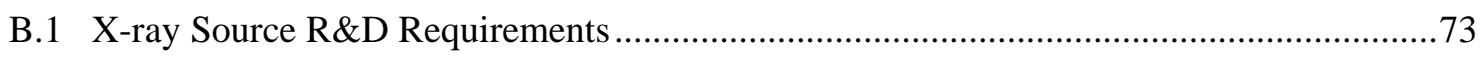

B.2 X-ray Optics R\&D Requirements.......................................................................... 76

B.3 X-ray Detector R\&D Requirements ....................................................................... 78

Appendix C: Interactions of X-Rays with Matter: Perturbative Limits and Mitigation Strategies...............81

C.1 Case Study: X-Ray Perturbation of a Ferromagnetic Metal ..............................................82

1 Response of the Metal on Femtosecond Timescales .................................................82

2 Response of the Metal on Picosecond Timescales.....................................................84

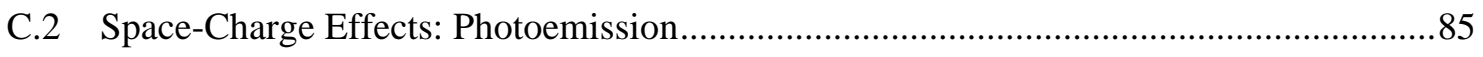

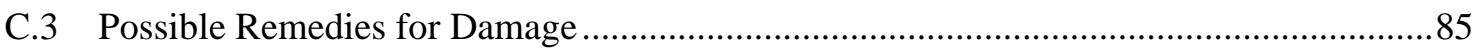

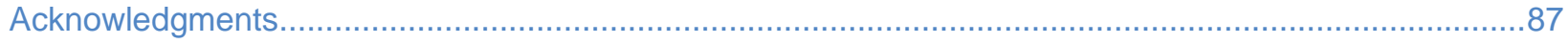




\section{Preface}

This document is the result of collaboration among scientists associated with Argonne National Laboratory (ANL), Brookhaven National Laboratory (BNL), Lawrence Berkeley National Laboratory (LBNL), and SLAC National Accelerator Laboratory (SLAC), including faculty of the University of California, Berkeley, and Stanford University. The collaboration consisted of scientists from a broad range of scientific disciplines and included experts in x-ray and accelerator science, two core competencies of these four Laboratories. The four Laboratories play a key role in the DOE Complex of National Laboratories, operating forefront $\mathrm{x}$-ray programs on the second-generation storage ring NSLS, the third-generation storage rings ALS, APS and SPEAR-3, and have forefront knowledge of advanced sources based on the construction of LCLS, the first hard x-ray laser, to be commissioned in 2009, and NSLS-II, an advanced third-generation storage ring to be commissioned in 2013. Information was also provided and coordinated with external experts and colleagues from around the world. 
2 WHITE PAPER 


\section{ONE}

\section{Introduction: Science and X-Rays}

Many of the important challenges facing humanity, including developing alternative sources of energy and improving health, are being addressed by advances that demand the improved understanding and control of matter. While the visualization, exploration, and manipulation of macroscopic matter have long been technological goals, scientific developments in the twentieth century have focused attention on understanding matter on the atomic scale through the underlying framework of quantum mechanics. Of special interest is matter that consists of natural or artificial nanoscale building blocks defined either by atomic structural arrangements or by electron or spin formations created by collective correlation effects (Figure 1.1).

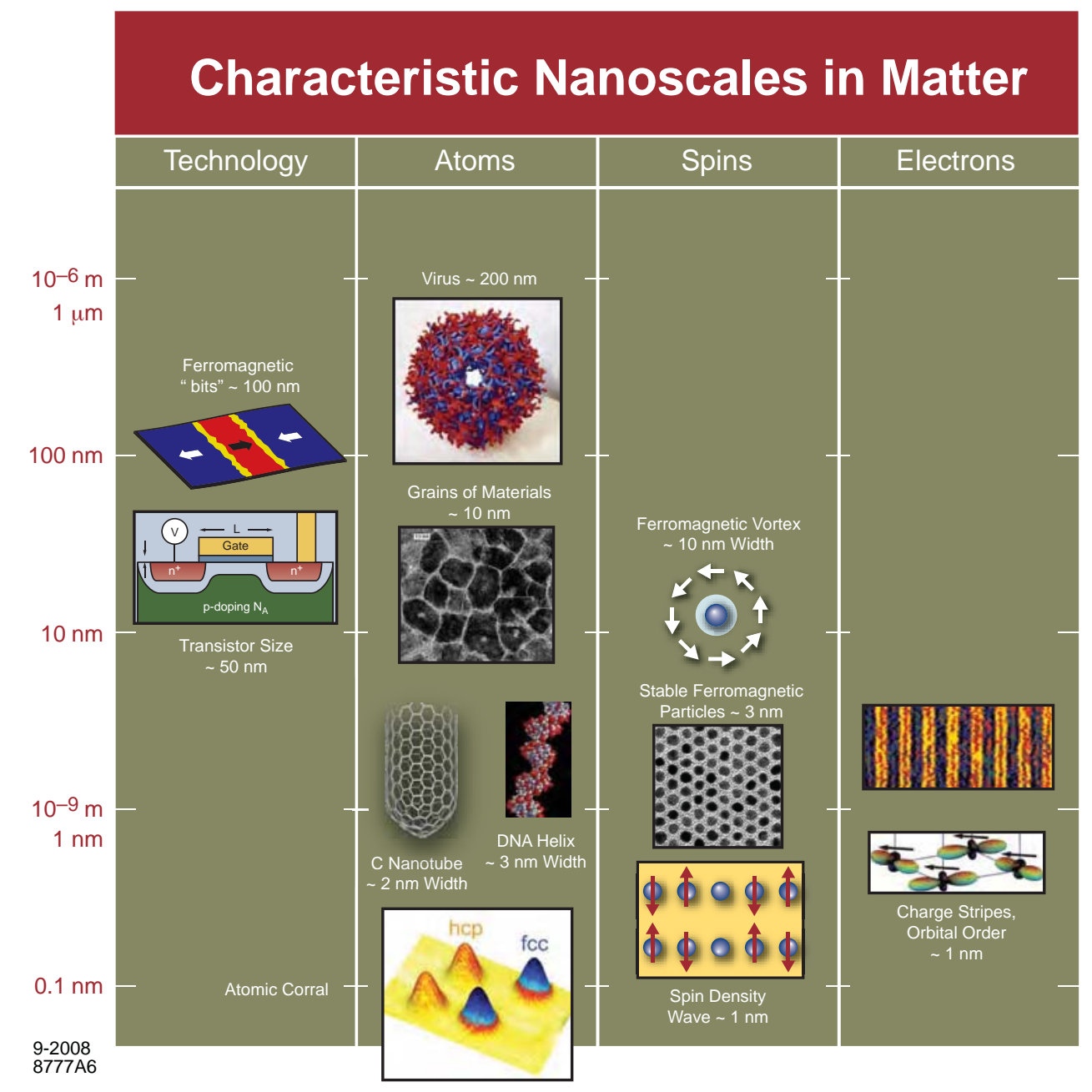

Figure1.1. Illustration of characteristic features on the level of atoms, electrons, and spins on nanometer length scales in comparison with typical lateral length scales in present advanced technological devices. 
The essence of the challenge to the scientific community has been expressed in five grand challenges for directing matter and energy recently formulated by the Basic Energy Sciences Advisory Committee $\left[{ }^{1}\right]$. These challenges focus on increasing our understanding of, and ultimately control of, matter at the level of atoms, electrons. and spins, as illustrated in Figure 1.1, and serve the entire range of science from advanced materials to life sciences. Meeting these challenges will require new tools that extend our reach into regions of higher spatial, temporal, and energy resolution.

X-rays with energies above $10 \mathrm{keV}$ offer capabilities extending beyond the nanoworld shown in Figure 1.1 due to their ability to penetrate into optically opaque or thick objects. This opens the door to combining atomic level information from scattering studies with 3D information on longer length scales from real space imaging with a resolution approaching $1 \mathrm{~nm}$. The investigation of multiple length scales is important in hierarchical structures, providing knowledge about function of living organisms, the atomistic origin of materials failure, the optimization of industrial synthesis, or the working of devices.

Since the fundamental interaction that holds matter together is of electromagnetic origin, it is intuitively clear that electromagnetic radiation is the critical tool in the study of material properties. On the level of atoms, electrons, and spins, $\mathrm{x}$-rays have proved especially valuable.

Future advanced x-ray sources and instrumentation will extend the power of x-ray methods to reach greater spatial resolution, increased sensitivity, and unexplored temporal domains. The purpose of this document is threefold:

1) summarize scientific opportunities that are beyond the reach of today's $x$-ray sources and instrumentation;

2) summarize the requirements for advanced $x$-ray sources and instrumentation needed to realize these scientific opportunities, as well as potential methods of achieving them; and

3) outline the R\&D required to establish the technical feasibility of these advanced $x$-ray sources and instrumentation.

\section{A. Brief History of X-Ray Science}

The understanding of matter was revolutionized by Röntgen's discovery of x-rays in 1895, and since that innovation, five invaluable and well-known uses of x-rays have emerged:

1) $x$-ray imaging, first demonstrated by Röntgen himself and widely known in medicine;

2) $x$-ray diffraction revealing the atomic structure of matter, pioneered by von Laue and the Braggs;

3) $x$-ray absorption and emission spectroscopy by Barkla, uncovering the complete electronic shell structure of atoms;

4) inelastic $x$-ray scattering, first demonstrated by Compton and later proven indispensable for measuring collective excitations and vibrational and elastic properties of matter and the magnetic properties and valence states of iron;

5) photoelectron spectroscopy revealing the signature of bonding in molecules and solids, pioneered by Siegbahn, Spicer, and Turner, followed by demonstration of its spin dependence by Siegmann.

${ }^{1}$ Directing Matter and Energy: Five Challenges for Science and the Imagination. Basic Energy Sciences Advisory Committee, U.S. Department of Energy, December 20, 2007. Available online at www.Sc.doe.gov/bes/reports/list.html. 
Before the advent of dedicated synchrotron radiation sources in the early 1970s, these applications of xrays had not only revolutionized our knowledge of matter on the fundamental level of atoms, electrons, and spins but also redefined entire fields of science, such as physics, chemistry, biology, and medicine. The 19 Nobel Prizes that have been awarded for related work, as summarized in Figure 1.2, prove the impact of x-rays.

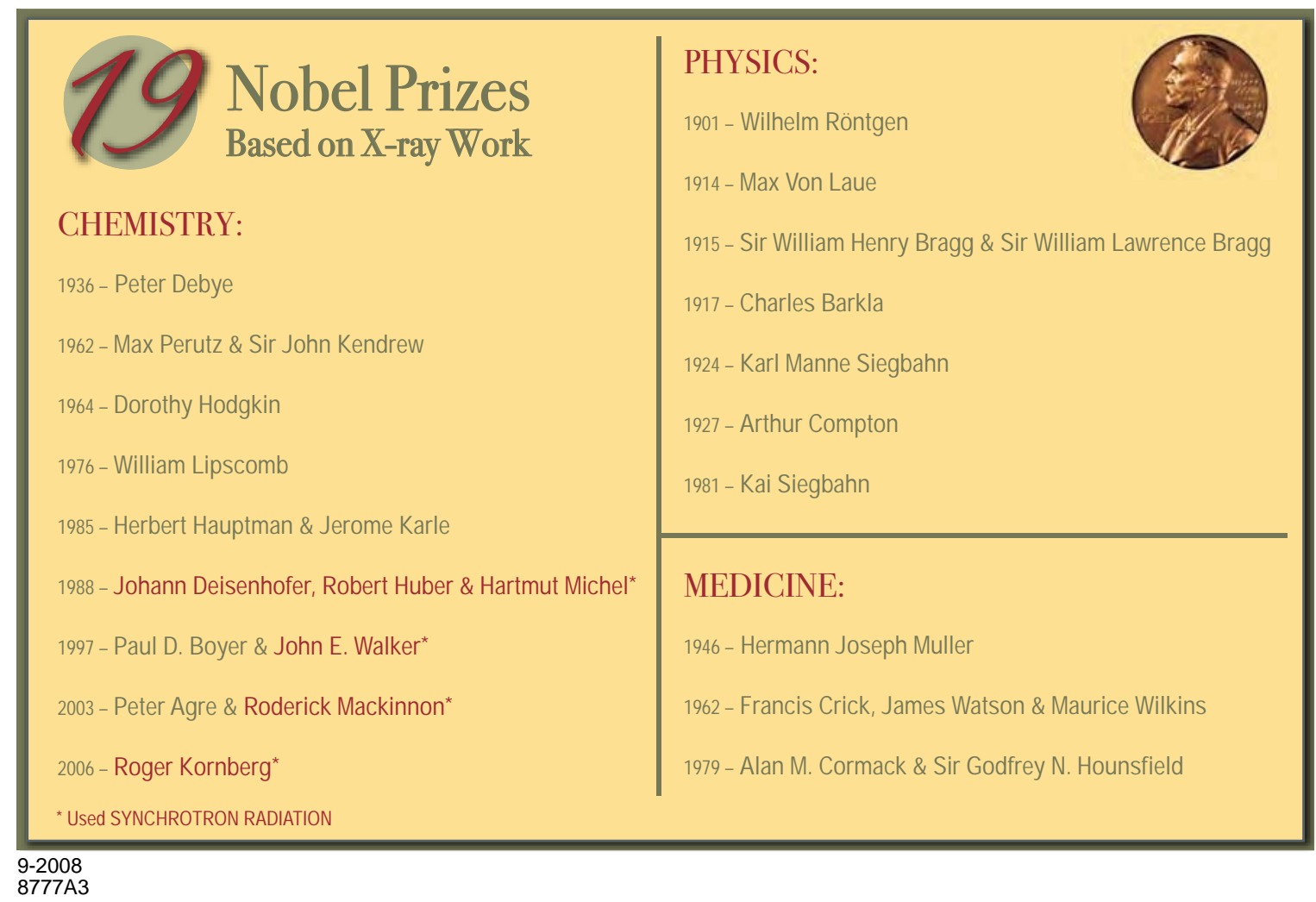

Figure 1.2. The impact of $x$-rays is proven by 19 Nobel Prizes that have been awarded for related work.

While most of these $\mathrm{x}$-ray accomplishments were based on the use of conventional x-ray tubes, as of today four Nobel Prizes can be linked to the use of x-ray synchrotron radiation. Based on the widespread application of synchrotron radiation for the solution of scientific problems, it is safe to predict that additional future Nobel Prizes will result from the use of synchrotron or FEL radiation..

\section{B. Modern X-Ray Techniques}

Key developments in x-ray science over the last thirty years are based on the unique properties of modern synchrotron radiation sources: high flux, high brightness and coherence, energy tunability and high energy resolution, polarization control, and pulsed time structure and associated instrumentation (imaging detectors with high spatial resolution and high efficiency, high-speed photon counting detectors, high accuracy optics for preserving source brightness and for focusing and analyzing the energy of the photons). The key capabilities of x-ray experiments in the light of the grand challenges are illustrated in Figure 1.3. 

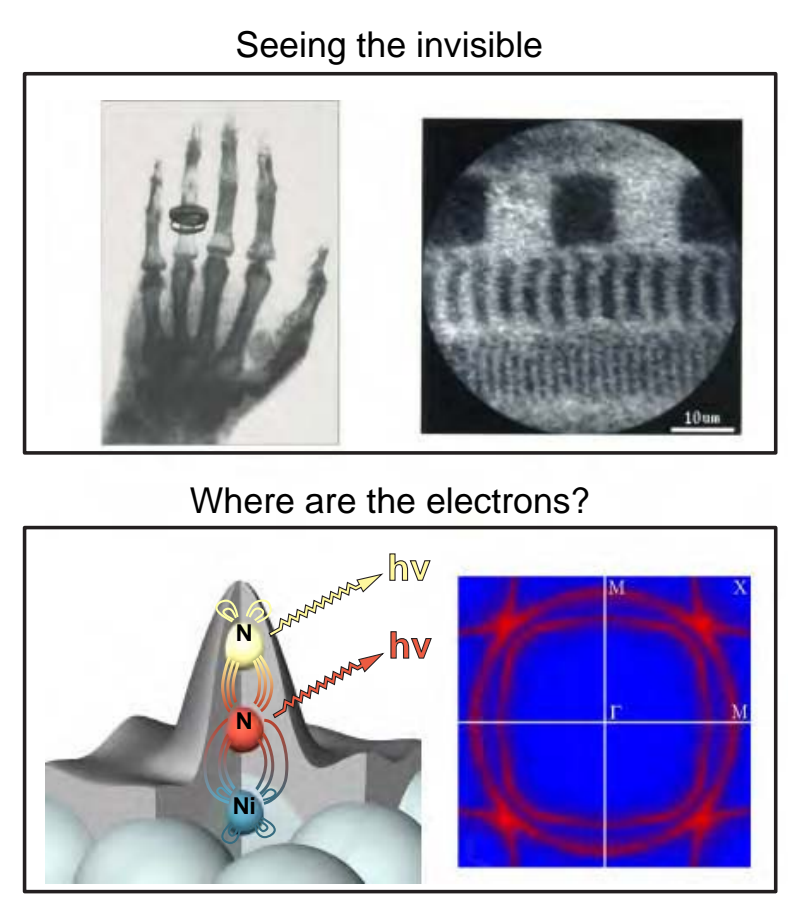
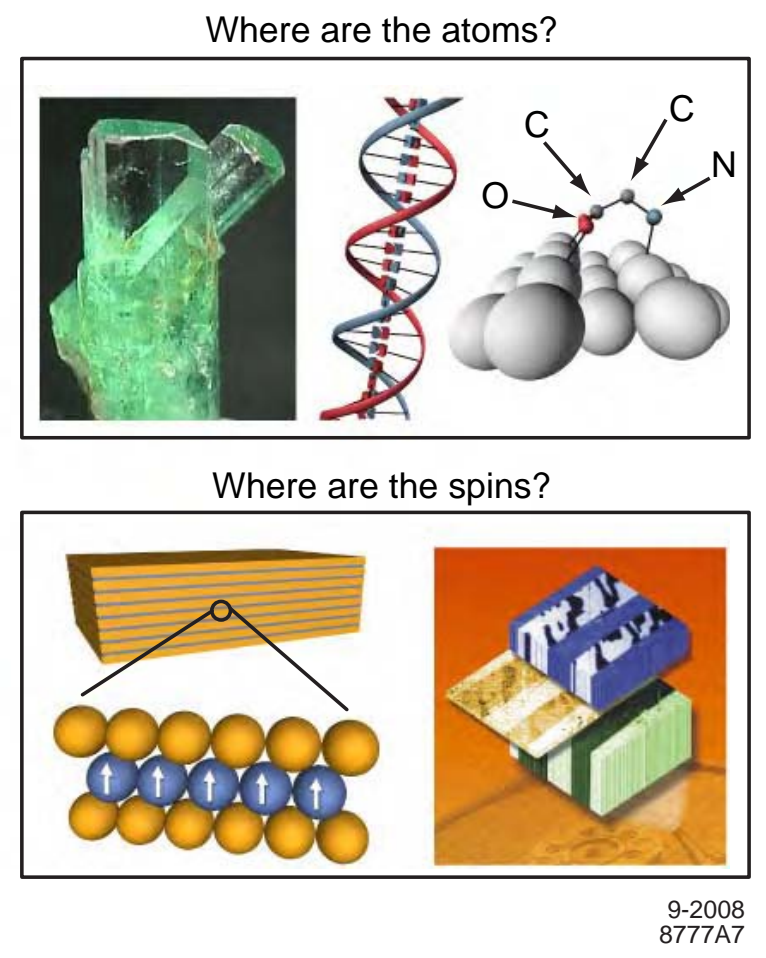

Figure 1.3. The basic capabilities of $x$-rays that provide the basis for the new developments discussed in the text.

X-rays penetrate matter and reveal the "invisible” interior of complex objects, such as living organisms. More recently in the era of nanoscience, $\mathrm{x}$-ray imaging, in the form of both real-space microscopy and inversion of reciprocal-space coherent scattering patterns ("speckle"), has provided high-resolution images on the nanometer length scale of charge and spin distributions in a sample. When combined with tunability and polarization control, x-rays can yield element- and chemical-state specific images together with magnetic contrast. Imaging techniques may be carried out both in a surface-sensitive mode with an electron microscope as in photoemission electron microscopy (PEEM) and in a bulk-sensitive mode by use of x-ray transmission. Microscopy is often carried out today in combination with pump-probe studies to reveal nanoscale dynamics on the tens of picosecond time scale with storage-ring sources and LCLS will extend this down to the 100 femtosecond timescale or less for hard x-rays. Coherent-scattering studies, carried out in a photon-correlation spectroscopy mode, are used to reveal equilibrium fluctuations on time scales ranging from milliseconds to thousands of seconds with existing storage ring sources. NSLS-II will extend this range down to sub-microseconds and LCLS will cover the range from nanoseconds to 100 nanoseconds with hard x-rays. The multi-dimensional combination of nanoscale spatial resolution with temporal resolution down to femtoseconds, even attoseconds, constitutes one of the greatest opportunities of future x-ray studies of matter. Finally, multiscale imaging on hierarchical systems is another key area. The improved spatial coherence and increased brightness promised by advanced x-ray sources will enable revolutionary science.

X-rays are often classified by their penetrating power which determines to a large extent the types of experiments that can be performed. Macromolecular crystallography, which makes use of x-rays from 3.5 $\mathrm{keV}$ to $20 \mathrm{keV}$ ) enables the determination of the atomic structure of matter. While the inversion of $\mathrm{x}$-ray diffraction patterns has long been used to give the real-space atomic structure of matter, about $80 \%$ of new 
macromolecular structures determined today by this method use synchrotron radiation. In particular, the techniques of multiple-wavelength and single wavelength anomalous diffraction (MAD and SAD) have revolutionized macromolecular crystallography. The most difficult and challenging problems, such as large complexes and molecular machines, are almost exclusively solved with synchrotron radiation. By use of coherent $x$-ray scattering, it is possible today to determine the three-dimensional atomic structure of nanometer-sized objects. Harder $\mathrm{x}$-rays $(20 \mathrm{keV}$ to $100 \mathrm{keV})$ are used, for example, for measurements that penetrate high pressure environments such as diamond anvil cells (DACs), or where it is essential to reduce sample damage from deposited $\mathrm{x}$-ray energy, or for phase contrast imaging in macroscopic technical materials. The tunability of the photon energy has led to the widespread use of $\mathrm{x}$-ray absorption in the form of the extended x-ray absorption fine structure (EXAFS) for the study of the precise atomic structure around selected atoms. A key component of future studies of matter with hard $\mathrm{x}$-rays is the combination of atomic resolution with temporal resolution on the sub-picosecond time scale of atomic motion.

The longer wavelength of lower-energy, soft $\mathrm{x}$-rays $(\sim 1 \mathrm{keV})$ enables the investigation of the electronic structure of matter. Soft $\mathrm{x}$-ray spectroscopic studies include near-edge $\mathrm{x}$-ray absorption fine structure (NEXAFS), x-ray emission spectroscopy (XES), and photoemission (PES). These techniques yield information on the electron distribution in occupied and empty orbitals in molecules and the electronic energy bands in solids, providing information on how atoms are bound together in solids, liquids, and molecules. Linear-polarization-dependent NEXAFS and XES studies have provided detailed information of directional bonding and orientational order at surfaces. Photoemission studies have revealed the detailed electronic structure of molecules and the spin-dependent band structure of solids, and angle-resolved photoemission spectroscopy (ARPES) has evolved into one of the most important tools in condensedmatter physics. Key future development areas of soft x-ray spectroscopic techniques are their extension to the multi-dimensional phase space of length scales down to nanometers, time scales down to femtoseconds, or even attoseconds, and energy scales to tens of micro-eV.

Not shown in Figure 1.3 are the recent developments in inelastic x-ray scattering that allow probing elementary excitations of the lattice as well as of the electronic system. This has become possible by coupling to the intrinsic linear momentum of high-brightness hard x-ray beams that are monochromatized to the meV scale. Similar to inelastic neutron scattering, such studies provide details of the excitations of the atomic lattice but are complementary to neutrons in emphasizing charge rather than spin excitations in the electronic system. A particularly important but as yet insufficiently employed variant of inelastic x-ray scattering uses core-electron excitations. Such x-ray Raman spectroscopy uses a penetrating beam of hard $\mathrm{x}$-rays and through "x-ray energy loss spectroscopy" provides spectra like $\mathrm{x}$-ray absorption for low-Z atoms in bulk samples that cannot be measured by means of strongly absorbing soft $\mathrm{x}$-rays. Examples are vacuum-incompatible samples like liquids and materials under extreme environments like those in diamond-anvil cells.

The angular momentum of circularly polarized $\mathrm{x}$-rays has allowed access to the angular momentum of electrons and consequently the ability to quantitatively separate the spin and orbital parts. X-ray magnetic circular dichroism (XMCD) studies preferentially use soft x-rays, where the magnetic effects are largest, for either $\mathrm{x}$-ray absorption spectroscopy or resonant scattering. Nanoscale information is obtained via realspace microscopy using zone-plate lenses or reciprocal-space coherent scattering ("lensless imaging"). For modern magnetic materials, which typically come in the form of thin films, multilayers, and nanostructures, such studies have replaced neutron scattering as the technique of choice because of their elemental specificity and sensitivity due to enhanced cross-sections (see Figure C.1 in Appendix C). 
While the above discussion amply demonstrates the power of x-rays for the study of matter, the question arises whether the capabilities of x-ray sources that exist today or are under construction are sufficient to address the full range of scientific challenges before us. Specifically, we note that control of matter will ultimately flow from understanding the structure and properties of the atoms and bonds that underlie matter and that yield an incredibly rich set of properties that range from superconductivity, to conversion of sunlight to electricity, to catalytic conversion of greenhouse gases, to miracle drugs.

We have concluded that the nation's scientific needs will not be met entirely by current facilities and those under construction, and in the next sections of this document we demonstrate that the scientific needs for new sources are clear and that the technological readiness to construct suitable sources is within our reach. 


\section{Overview of Scientific Drivers and Required Enhanced X-Ray Capabilities}

In this section we give a brief overview of the scientific challenges associated with the understanding and control of matter at the level of atoms, molecular assemblies, electrons, and spins in the domains of space, time, and energy, and we outline five key x-ray capabilities that are required to address the scientific challenges. A more detailed discussion of the science drivers will be given in Section 3, and the basic types of x-ray sources that can provide the required capabilities are discussed in Section 4.

\section{A. Scientific Drivers}

While there is compelling historical evidence that the construction of new x-ray sources by itself has served as scientific enablers leading to originally unimagined applications, here we take the approach of using identified scientific challenges to demonstrate a need for future x-ray capabilities.

The innovation expected from the construction and operation of enhanced x-ray sources lies in combining and extending existing knowledge of the nanoworld, illustrated in Figure 1.1, with extreme time and energy resolution. Properties of anticipated new x-ray sources include the ability to reach to the frontier of ultrafast timescales of electron motion around an atom, the spatial scale of the atomic bond, and the energy scale of the bond that holds electrons in correlated motion with near neighbors. X-ray sources offer unique capabilities to combine information on atomic length scales with data on full-scale functional systems under realistic operational conditions. In addition, these novel sources have an intensity and brightness needed to observe the subtlest of nature's secrets at these frontier space, time, and energy scales.

While our understanding of the time- and energy-dependent behavior of matter in its ground or natural state is still severely limited, we know even less about excited states. Such states are of key scientific and technological importance, for they typically determine how matter functions during chemical reactions and during physical and biological processes. Excited states of interest span a vast range, being close to the natural (ground or equilibrium, as appropriate) state (as in electronic transport), relatively far from it (as in photo-chemical reactions), and very far from it (as in extreme conditions that can be imposed by pressure, radiation, or electric and magnetic fields).

To briefly illustrate the need for advanced x-ray capabilities, two examples are given below of what we need to learn. They are related to "chemical reactivity" and "complex materials," two of the five important scientific areas that are discussed in more detail in Section 3.

- Knowing the importance of energy in guiding chemical reactions, we envision driving chemical transformations by controlled optical or infrared pulses and understanding the atomic and electronic transformation by means of advanced x-ray spectroscopies. More specifically, we need to capture, with snapshots on the femtosecond timescale, the making and breaking of chemical bonds and the crucial transition-state intermediates in chemical reactions. 
- We foresee an understanding of the origins of nanoscale charge, spin, and orbital order and their dynamics in correlated materials through high-resolution energy- and time-dependent $\mathrm{x}$-ray tools with nm resolution. The correlated interactions lead to unusual phenomena such as high-transition temperature superconductivity, metal-insulator transitions and novel phases. We need to visualize through ultrafast x-ray motion pictures the performance limits of materials and electronic devices, e.g., the speed limit of a spintronics device.

\section{Characteristic Times in Matter}

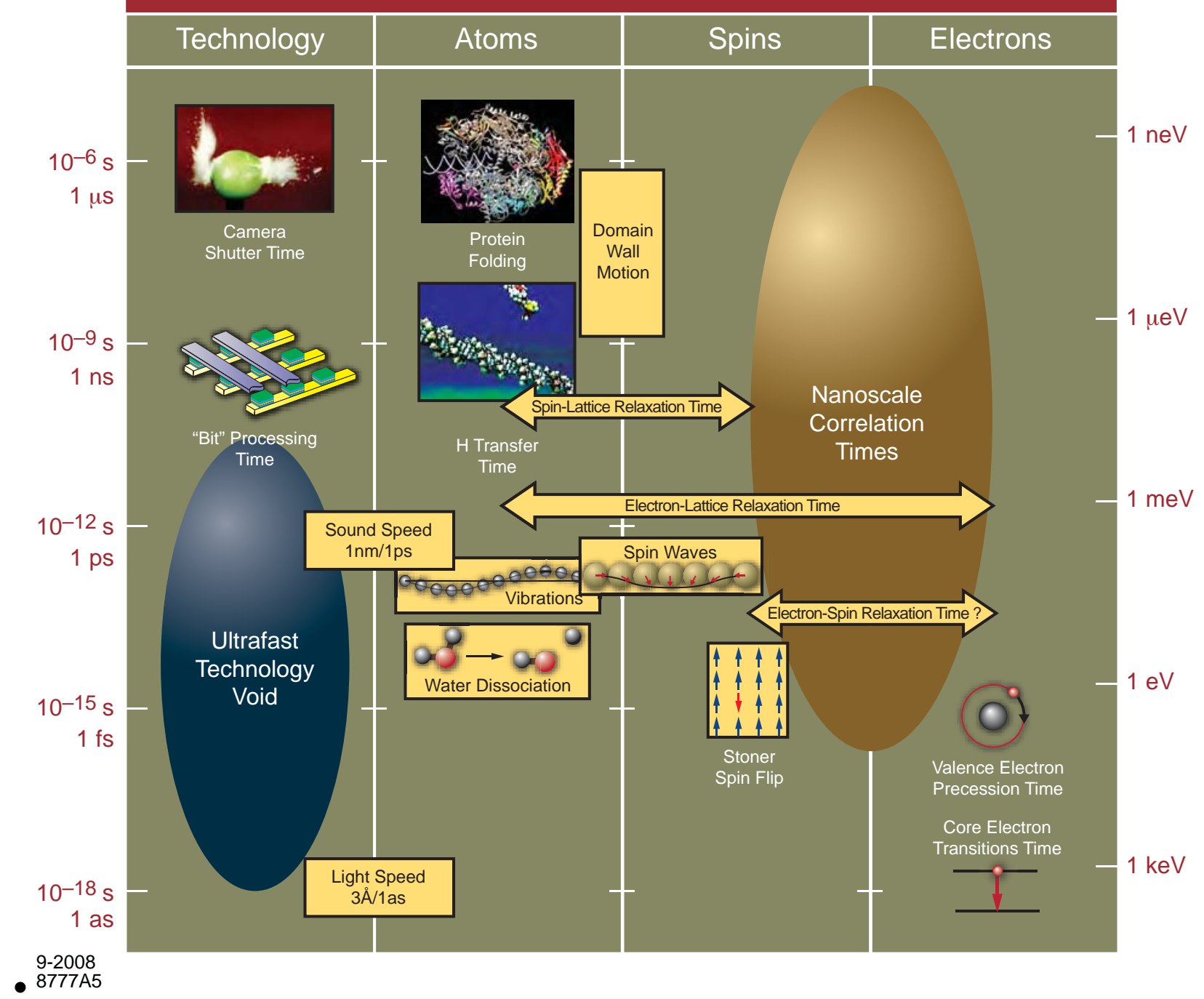

Figure 2.1. Illustration of our present knowledge about typical times involved in the interactions of atoms, electrons, and spins. On the right, we have indicated the corresponding quantum-mechanical interaction energies as estimated from the energy-time correlation $\Delta \mathrm{E} \cdot \Delta \mathrm{t}=\mathrm{h} \sim 4 \mathrm{fs} \cdot \mathrm{eV}$.

Embedded in these challenges is the exploration of the atomic or nanoscale on the "natural" time scale of atoms, electrons, and spins and on the "operational" time scale that determines function and is the key in technological applications. As illustrated in Figure 2.1, there is presently a striking discrepancy between the natural time scales of atomic motion (about $100 \mathrm{fs}$ ), spin motion (down to about $1 \mathrm{fs}$ ), and electronic 
motion (down to attoseconds) and the fastest operational timescales (approaching $100 \mathrm{ps}$ ). A critical scientific challenge is to pave the way for technology to expand into the ultrafast, with the opportunity of five orders of magnitude improvement down to the intrinsic time scale of charge and spin motions of valence electrons.

The unique strength of future $\mathrm{x}$-ray sources lies in their ability to combine coverage of the atomic and nanoworld with the entire time domain from seconds to attoseconds and a spatial domain from $0.1 \mathrm{~nm}$ to $\mathrm{mm}$. Section 3 provides more specific examples of scientific challenges in different areas of science that call for enhanced x-ray capabilities.

\section{B. Essential New X-Ray Capabilities}

In addition to the time scales, the length scales associated with functional systems on both short and longer time scales are also important. These are the longer time scales characteristic of diffusion and displacement, and of sound propagation, for example. Higher coherent flux from future x-ray sources will permit imaging on time scales of $\mathrm{MHz}$. This opens the door to scientific discovery in catalysis, deformation, phase nucleation and transformations.

Here we briefly describe four key performance areas where significant advances in x-ray capabilities are both required by the science, and technically feasible in the near-term. Not only are these individual requirements beyond the capabilities of present light sources, the paramount scientific challenges often require a source providing several of these features simultaneously.

\section{X-Ray Time Structure-Complete Control of Longitudinal Phase Space}

- Pulse duration and temporal resolution extending to the attosecond regime.

- Fourier-transform-limited pulse structure extending from few-picosecond pulses with meV bandwidths to sub-femtosecond pulses with $10-\mathrm{eV}$ bandwidths.

- Control of longitudinal pulse shape, amplitude and phase.

- Synchronization and full integration with conventional pulsed laser sources.

Access to fundamental time scales (and energy scales) combined with atomic resolution, element specificity, valence-electron sensitivity, etc. is a requirement that emerges throughout the scientific grand challenges. Initial work at third-generation synchrotrons has launched this research area, and firstgeneration free electron lasers (FELs) will provide an important advance in this emerging field. A key missing capability is longitudinal coherence (and pulse control) that is achievable using advanced seeding or oscillator techniques and is essential to maximize the scientific impact of future short-pulse x-ray sources. Concurrently "conventional" laser sources must be synchronized and fully integrated with future short-pulse $\mathrm{x}$-ray sources in order to provide for tailored ultrafast sample excitation spanning the UV, visible, infrared, and THz regimes. Next generation FELs will also deliver transform-limited x-ray pulses of $0.1 \mathrm{meV}$ energy resolution with unprecedented intensity allowing one to probe dynamics of electrons, spins, phonons and other quasi-particle excitations in condensed matter ultimately with a few nm spatial resolution. On the other hand, the real-time methods will allow access to critical dynamics of large conformational motions in chemical reactions or proteins with $\mu \mathrm{eV}$ (nanoseconds) to neV (microseconds) 
resolution. Presently such studies are performed with no spatial resolution using spin-echo neutron scattering or quasi-elastic nuclear resonant scattering of x-rays.

A future promise is the extension of optical frequency comb techniques to the VUV and x-ray regions with the development of mode-locked seeded x-ray FEL pulses. The new frequency resolution from this approach will be unprecedented in the x-ray range and ultrahigh resolution metrology capability may permit applications to cosmology. For example, one may be able to speculate on the measurement of time variation of the fundamental constants of nature, symmetry postulates, graininess of space and gravitational redshift.

\section{Full Transverse Coherence}

- Requirements set by real-space imaging, diffractive imaging, and photon-correlation spectroscopy.

- Optical systems to preserve and exploit transverse coherence.

Present ring-based sources provide diffraction-limited radiation in the vertical plane up to several keV and in the horizontal plane up to $100 \mathrm{eV}$ (see Figure 4.3 below). Extending these capabilities to higher photon energies (at high average brightness) is essential for performing ultimate reciprocal-space diffraction experiments with atomic resolution as well as advanced x-ray imaging of nanostructures, domains, and emergent phenomena that are manifest through phase separation, domain fluctuations, etc. Future sources promise the measurement of the entire range of spatial length scales from $0.1 \mathrm{~nm}$ to $1 \mathrm{~mm}$. At the same time, enhanced peak-coherent flux will allow time-domain measurements to be extended from seconds to femtoseconds. The measurements with future femtosecond pulse x-ray sources with high peak brightness and $\mathrm{MHz}$ pulse repetition rates might even mitigate issues related to sample damage.

\section{High Average Flux and Brightness}

- Quasi-CW (up to $1 \mathrm{GHz}$ ) sources with average flux and/or brightness substantially beyond existing sources in the hard and/or soft x-ray range.

- Short-pulse sources with high repetition rates (up to $1 \mathrm{MHz}$ ) providing average flux and/or brightness substantially beyond existing sources in the hard and/or soft x-ray range.

The tremendous scientific promise of x-ray Raman, inelastic x-ray scattering, nuclear resonant scattering and related x-ray tools is significantly limited by the spectral flux (photons/bandwidth) available from present $\mathrm{x}$-ray sources in both the hard and soft x-ray ranges. The scientific impact of present short-pulse $\mathrm{x}$-ray sources is similarly limited by the available peak and average flux and brightness. Furthermore, time-resolved experiments and nonlinear x-ray science at short-pulse sources require the ability to tradeoff peak and average brightness for specific applications. Imaging experiments at very high frame rates (beyond MHz) will be made possible by future high average brightness sources such as ERLs, FELs, or super storage rings.

\section{Tunability, Polarization Control, and Extended Photon Energies}

- Soft x-ray tunability (throughout the transition-metal L-edges) and polarization control and modulation.

- Hard x-ray tunability and energy range extending to $100 \mathrm{keV}$. 
While tunability and polarization control are well developed at third-generation synchrotron sources, highsensitivity measurements with future sources require the ability to modulate the polarization at high frequencies $(\mathrm{kHz})$, a capability presently not available. Polarization control will also be an important capability at future short-pulse x-ray sources, both in the soft and hard x-ray range, to exploit powerful spectroscopy techniques (e.g., NEXAFS, XMCD, x-ray magnetic linear dichroism or XMLD, magnetic scattering, nuclear resonance scattering, etc.) for probing valence charge, spin, bonding dynamics, phonons and emergent phenomena on fundamental time and spatial scales. Tunability is essential across the entire spectral range for elucidating the dynamics of local atomic structure (e.g., via EXAFS, anomalous scattering, etc.), providing element specificity, penetration capability, interface sensitivity, etc. 



\section{Examples of Scientific Drivers for Future X-Ray Sources}

This section presents examples of scientific challenges in five areas where advanced x-ray capabilities are envisioned to contribute to breakthroughs in our knowledge. The chosen areas are:

1) atomic and molecular science - studying fundamental processes involving atoms, electrons and spins;

2) chemical reactivity - discovering the key far-from-equilibrium processes related to energy production, climate change, and environmental processes;

3) complex materials - a core subject of condensed-matter physics and engineering sciences, which often drive technological advances - and their materials synthesis;

4) materials under extreme conditions - revealing new synthesis pathways of tailored matter and discovering the limits of materials stability, performance, and function;

5) life sciences and soft matter-studying the structure and dynamics of organic building blocks of technological materials_promising the improvement of health.

While the above topics were chosen to cover key societal issues, our discussion is necessarily incomplete. It could be easily extended to other scientific and engineering areas. Furthermore, we believe that future $\mathrm{x}$-ray facilities will enable research on yet unidentified scientific and technological topics that will emerge over the next two decades. The present section should be read with this understanding.

\section{A. Understanding and Controlling Electronic, Atomic, and Molecular Dynamics on Their Natural Timescales}

The first of the challenges in the BES grand challenges report is "Quantum control of electrons in atoms, molecules, and materials." This issue is now a central one for the scientific community because we are at a point of transition in our study of the most basic questions in chemistry and materials science. For a century we have focused on how electrons, which are the "glue" of chemistry, determine the properties of materials and direct chemical and physical changes. We are now at the dawn of the new science of quantum control where we will go beyond probing what is there in molecules and materials to controlling processes through the direct manipulation of the electrons. Our primary tools for this task are short pulses of electromagnetic radiation that were made possible in recent decades by the advent of ultrafast laser pulses in the infrared (IR), visible, and ultraviolet (UV). Powerful, ultrafast pulses in the extreme ultraviolet (EUV) and x-ray regions form the other half of the tool kit that will open the doors to a complete and successful experimental attack on this most fundamental of challenges.

At stake are the solutions to some of the world's most important technological problems where quantum physics and chemistry play a central role. How can we produce enough energy? How can we make computers smaller, faster, and cooler, and what are the true limits of computation? How can we detect harmful pathogens early when their concentrations are almost vanishingly small? Most fundamentally, how can we make quantum-scale systems work for us? To answer these questions we will have to go 
beyond the simple pictures of electron orbitals and the separation of the timescale of electron motion from that of the nuclei in a molecule, on which, until now, our physical and chemical intuition have been based.

\section{Attosecond Manipulation and Control of the Correlated Motion of Electrons}

In the valence shells of atoms and molecules, where bonding forces are created, the natural timescale of the motion of electrons responsible for generating the most important properties of molecules is of the order of a few hundred attoseconds. Recently, sub-femtosecond pulses in the soft x-ray regime have opened up the possibility for direct study and manipulation of electronic motion on that timescale. The experiments that will do so in the future are dramatically more challenging than those being undertaken today, in terms of both detection and combinations of multiple radiation pulses, and will rely on the robust detection of signals from effects that are almost undetectable today. To be successful, they will require flexible and intense $\mathrm{x}$-ray sources that provide those signals and that will allow multicolor $\mathrm{x}$-ray experiments.

The essential idea of these experiments is to catch electron dynamics in the act during transitions between electronic states caused by any perturbation. One class of experiments will first pump an atom or molecule into an excited state, for example an autoionizing state with a finite lifetime, and then probe it with a subfemtosecond pulse during that lifetime to ionize or doubly ionize the target. These experiments will make use of momentum-imaging detectors of the electrons and ions produced in coincidence to give a complete momentum map of the final state, a map in which the signatures of correlation in the initially pumped metastable state and its decay are written. Other experiments will probe transitions between electronic states caused by the motion of nuclei in molecules when those electronic states are close in energy. Experiments such as these will push the limits of FEL sources, since they require: tunable and intense subfemtosecond pulses, synchronization with other sources (lasers, high-harmonic generation or another FEL pulse), and high repetition rates.

\section{Understanding Chemical Dynamics When the Timescales of Nuclear and Electronic Motion Can Coincide}

While the natural timescale of electron dynamics is of the order of a few hundred attoseconds, that of the motion of the atoms themselves during a reaction or as they vibrate in a molecule is of the order of tens to hundreds of femtoseconds (see Figure 2.1). However, when the nuclei in a molecule are near the molecular geometries where two electronic states have the same energy, the collective motion of the electrons as they make a transition from one state to the other can match the slower timescale of the motion of the nuclei. This phenomenon, shown schematically in Figure 3.1, is commonly called the "breakdown of the BornOppenheimer approximation.” Up to now, this approximation has provided the conceptual basis for most of our understanding of chemical reactions. The grand challenges report identifies this phenomenon as a central challenge to our understanding of photosynthesis and the photochemistry of the first step in vision, for example. The surprise is that in large molecules involved in photochemical processes like photosynthesis, there are generally many such intersections, and nature has made use of them to control the flow of energy and chemical change.

X-ray FELs will open new doors to understanding and exploiting these phenomena. In conventional femtosecond photochemistry, a vibrational wave packet is created within a single electronic excited-state and the subsequent molecular dynamics are determined by the Born-Oppenheimer potential-energy surfaces on which the wave packet evolves. The advent of extreme ultraviolet (EUV)/soft x-ray attosecond 
pulses affords access to a new excitation regime that cannot be described within the Born-Oppenheimer approximation. For example, a 300-as pulse has a bandwidth of $6 \mathrm{eV}$, sufficient to create a novel type of electronic wave packet, i.e., a coherent superposition of multiple electronic states. The possibility of determining the character of that wave packet opens an entirely new dimension for molecular control. For example, the coherent interaction of electronic wave packets at conical intersections has the potential to control the branching between electronic states. Phase and amplitude shaping of the attosecond excitation pulse enables complete manipulation of the initial electronic (and vibrational) wave packet and its evolution. Attosecond $\mathrm{x}$-ray probe pulses will be essential for following the electronic and molecular dynamics via time-resolved photoelectron spectroscopy and/or x-ray absorption spectroscopy techniques.

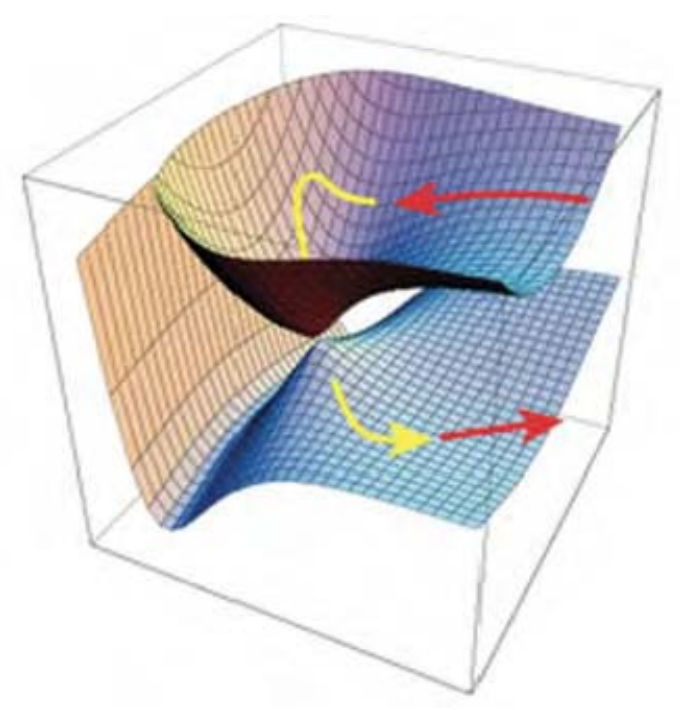

Figure 3.1. Example of a conical intersection, the point of contact between the potential-energy surfaces of two different electronic states in a molecule. [Source: BESAC grand challenges report]

\section{Detecting the Correlated Motion of Electrons with a New Kind of X-Ray Spectroscopy}

In the past decade, dramatic advances have been made in optical correlation spectroscopy, a descendant of transient four-wave mixing, in which carefully timed sequences of femtosecond pulses are used to probe the vibrational and electronic structure of matter. This approach is analogous to multidimensional nuclear magnetic resonance (NMR) spectroscopy, which probes the atomic structure of small molecules and for which the 1991 Nobel Prize in Chemistry was awarded. Optical correlation spectroscopy provides a powerful tool for studying how electronic and vibrational states interact, but it cannot access the temporal evolution of the valence change density directly.

The prospect of this kind of correlation spectroscopy in the x-ray region suggests the possibility of a revolution in our understanding valence charge structure, correlation, and its evolution, particularly in complex molecular systems and correlated materials. Major advantages are element specificity, subfemtosecond resolution, and unrestricted access to the valence states. X-ray correlation spectroscopy exploits core-level resonances (rather than homo-lumo transitions) with coherent attosecond pulses, and directly probes the dynamic correlation between valence charges associated with different atomic sites. These electron correlation effects are ubiquitous in chemistry; they are comparable in magnitude to 
chemical-bonding energies and are thus crucial for predicting molecular geometries, reaction barriers, and reaction rates with chemical accuracy.

Figure 3.2 illustrates the concept of x-ray correlation spectroscopy applied to a simple molecule, aminophenol. The goal is to detect the correlation of electronic excitations associated with the oxygen atom with those associated with the nitrogen and to follow this coupling on the natural time scale of electron motion. Attosecond pulses at $400 \mathrm{eV}$ and at $535 \mathrm{eV}$ initiate $1 s$ core excitations in $\mathrm{N}$ and $\mathrm{O}$ atoms, respectively, in a sequence of three x-ray pulses separated by times $t_{1}$ and $t_{2}$. This sequence generates a coherent four-wave mixing signal $S\left(t_{3}, t_{2}, t_{1}\right)$ that is measured in amplitude and phase via heterodyne detection. The two-dimensional Fourier transform of this signal with respect to $t_{1}$ and $t_{3}$ yields a twodimensional electronic spectrum in frequency space. Off-diagonal features in this two-dimensional spectrum are present only when there is correlation between the two excited electrons on the $\mathrm{N}$ and $\mathrm{O}$ atoms. Calculations show that the extent of this correlation depends not only on molecular structure but also on the nature of the molecular orbitals excited within the energy envelopes $(\sim 10 \mathrm{eV})$ of the incident pulses.
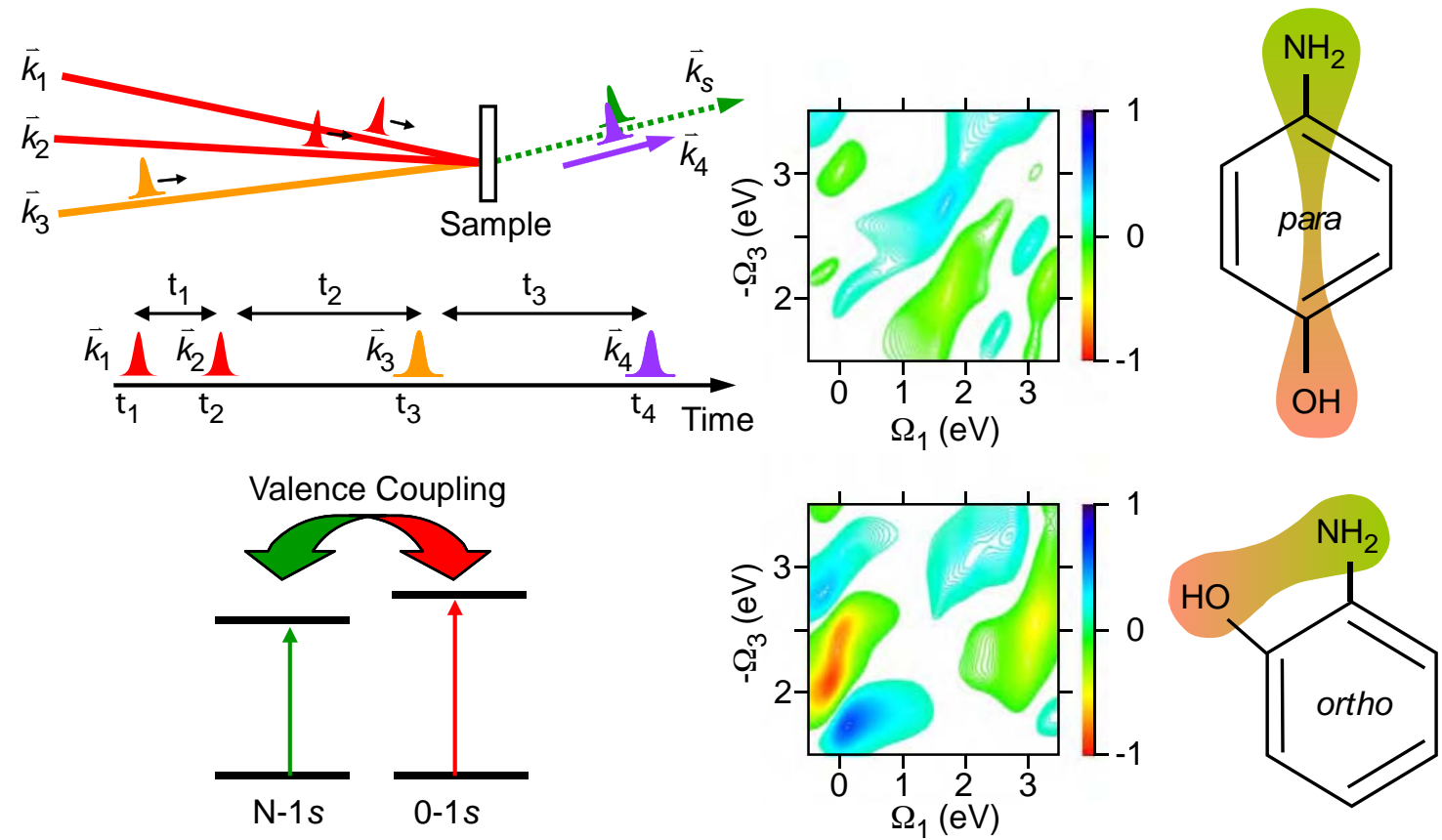

9-2008

$8777 \mathrm{~A} 14$

Figure 3.2. Left: Proposed four-wave mixing of ultrashort $\mathrm{x}$-ray pulses resonant with the O-1s and N-1s levels; Middle: theoretically predicted two-dimensional spectra the lower of which exhibits the coupling of excitations on the oxygen with those of the nitrogen in para and ortho-aminophenol molecules at right. [Source: S. Mukamel]

\section{What Is Needed?}

The experiments of the next two decades will make use of a variety of pump-probe configurations in which an ultrafast $\mathrm{x}$-ray probe will interrogate a system by chemically selective ionization following excitation by a short pulse in the visible, UV, or XUV. New FEL facilities in the soft and hard x-ray will have to provide exquisite synchronization with ultrafast laser sources providing short pulses from the XUV to the far IR region. Other experiments will require two-color $\mathrm{x}$-ray pump-X-ray probe capabilities. Thus the synchronization of two different FEL pulses will also be an essential characteristic of these new 
sources. Experiments in which electrons or ions are detected will demand high repetition rates, while experiments exploiting nonlinear x-ray optics will demand longitudinal coherence and control. It is expected that ultimate frequency metrology experiments in the soft and hard x-ray ranges will be possible with the development of frequency combs using $\mathrm{MHz}$ repetition rate pulses from oscillator or seeded FELs.

\section{B. Chemical Reactivity: From Nature's Catalysts to Controlled Reactions}

Understanding and controlling chemical reactivity at the scale of atoms and electrons will have a profound impact on many areas of human endeavor. Examples include sustainable energy production and storage, new materials, environmental and atmospheric science and remediation, and human health. Arguably the most important chemical reactions and those most difficult to understand and control occur in liquids or solids, and especially at surfaces or interfaces between solids and liquids. These molecular reactions underlie essential processes in chemical or biological catalysis, solar energy harvesting by natural and artificial systems, energy storage and transport, and biochemical processes. Moreover, life itself is based on the chemistry in heterogeneous aqueous environments.

The main challenges for chemical reactivity in such systems are: (1) to understand the interplay between electronic structure (valence charge distributions, energy levels, bonding, spin) and atomic arrangement (bond distances and angles, coordination), and (2) to control the course of the reactions. Molecular transition states, which are intermediate between reactant and product species, are of particular significance in catalytic reactions. In this transient regime, subtle conformational changes and chemicalbond formation and dissolution ultimately determine the reactive pathways. Understanding and identifying the various reaction pathways and the energetics of the intermediates involved in the reactions is critical for improving catalysts. In order to arrive at this deeper understanding, we must be able to observe the reactions in real time and identify the transient chemical species on timescales ranging from milliseconds to femtoseconds. Both the kinetics and dynamics of a reaction step within the sequence are critical, and new x-ray sources are needed to achieve this goal.

\section{Understanding Light-Induced Catalysis_Learning from Nature}

Nature uses remarkably varied systems and mechanisms to perform catalytic reactions with extraordinary efficiency, speed, and complexity. At the active site of many enzymes is a metal center with just a few atoms that is responsible for the rearrangement of electrons and atoms needed to carry out the catalytic reaction. For example, the light-induced oxidation of water to dioxygen during photosynthesis by green plants and cyanobacteria is a process that occurs on a large scale in the biosphere and is essential for life on Earth. Using energy from sunlight, manganese centers in cyanobacteria and plants can split water and evolve $\mathrm{O}_{2}$ at a rate of almost 1000 molecules/sec, three orders of magnitude faster than we can viably accomplish in the laboratory today. This sunlight-driven reaction couples the one-electron primary charge separation (photo-oxidation) with the four-electron water-oxidation chemistry. The electron extracted from water can be used for the reduction of $\mathrm{H}^{+}$to $\mathrm{H}_{2}$ or for $\mathrm{CO}_{2}$ reduction (see Figure 3.3). This natural catalytic scheme motivates design and synthesis of artificial photosynthetic systems based-on mixed-valence transition-metals bridged by oxygen and driven by visible light to produce carbon-neutral fuels from $\mathrm{CO}_{2}$ 


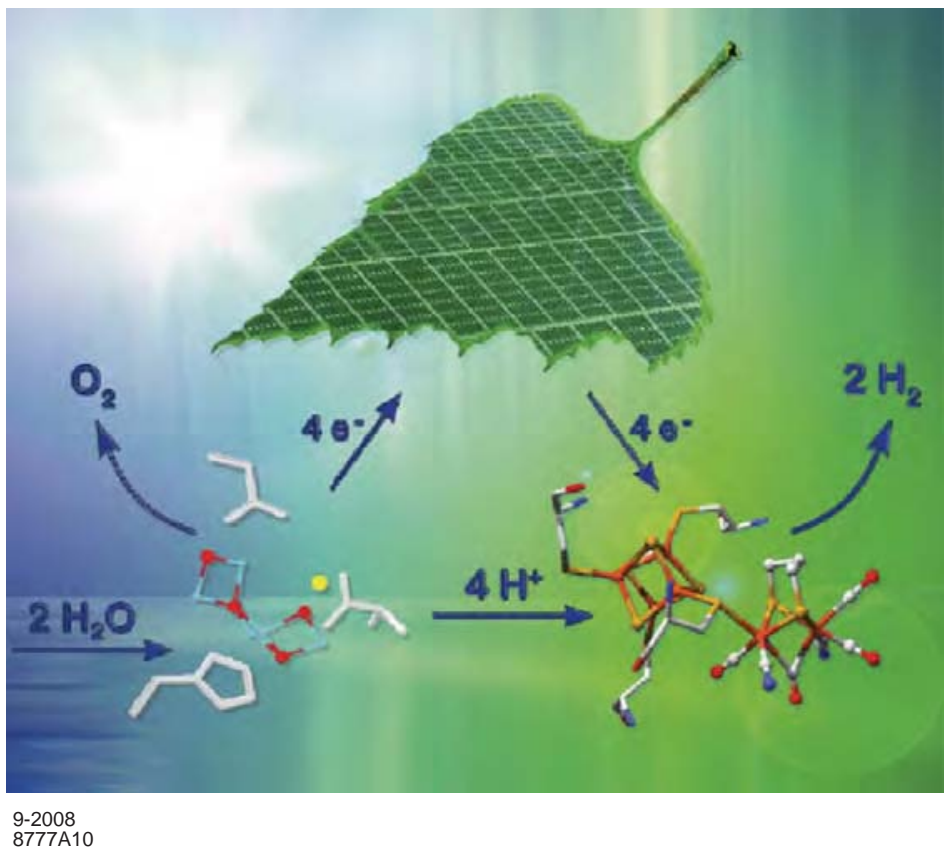

Figure 3.3. Photosynthetic water splitting and a hydrogenase powered by a lightharvesting antenna complex. [Source: W. Lubitz, E.J. Reijerse and J. Messinger, Energy Environ. Sci. 1, 15 (2008)]

and water. However, the development of economically feasible photo-catalysts requires a much better understanding of how these complex molecular systems function.

Much of the current progress in understanding the geometric and electronic structure of these systems on an atomic level is based on x-ray crystallography and spectroscopy studies (EXAFS, NEXAFS, XES) of reactant-states or intermediate-states trapped at low temperature following photo-excitation. However, our understanding of both natural and artificial photosynthetic systems is significantly limited by our inability to probe the molecular structure (with atomic resolution) and to follow the valence charge distribution and oxidation states under ambient conditions and on their natural times scales, whose range covers femtoseconds (characteristic of energy transfer and charge separation), $100 \mathrm{fs}$ (characteristic of molecular vibrations, bond formation/dissolution), picosecond timescales (characteristic of molecular conformational changes and diffusive processes), and micro and milliseconds (reactions in complex biocatalysts). In addition to the need for time-resolved crystallography and x-ray spectroscopy studies, proposed multidimensional x-ray spectroscopy techniques may have a tremendous scientific impact by enabling us to follow both the coherent and incoherent flow of valence charge between different active metal sites in catalytic complexes. All of these studies are beyond the capability of present x-ray sources, and yet they promise to have profound consequences for understanding complex molecular systems.

\section{Real-Time Catalysis and Surface Chemistry}

Heterogeneous catalysis provides a compelling example of both the scientific challenges and the impact of understanding and controlling chemical reactivity. A vast number of important processes rely on catalysis on surfaces, and their significance is evidenced by the 2007 Nobel Prize in Chemistry. Nevertheless, much 
needs to be learned about the intermediate steps of catalytic reactions at surfaces. In particular, we currently have very little knowledge of the dynamics of elementary processes on ultrafast timescales. Figure 3.4 illustrates the various steps of a typical surface reaction including adsorption, formation of different intermediates, and desorption. During each reaction step, there are rapid charge and energy transfers between the different adsorbates and the catalytic substrates that determine the dynamics and thereby the overall efficiency of the sequence. Examples of important catalytic reactions that involve dissociation of the strong internal $\mathrm{N}_{2}, \mathrm{O}_{2}$, or $\mathrm{CO}$ bonds as a rate-limiting step include ammonia synthesis for fertilizer production, and Fischer-Tropsch synthesis of fuels and oxygen reduction in fuel cells. The dissociation steps are strongly exothermic, and the dynamics of the energy dissipation between the final products and the substrate, where potentially large non-adiabatic processes can occur, is not understood.

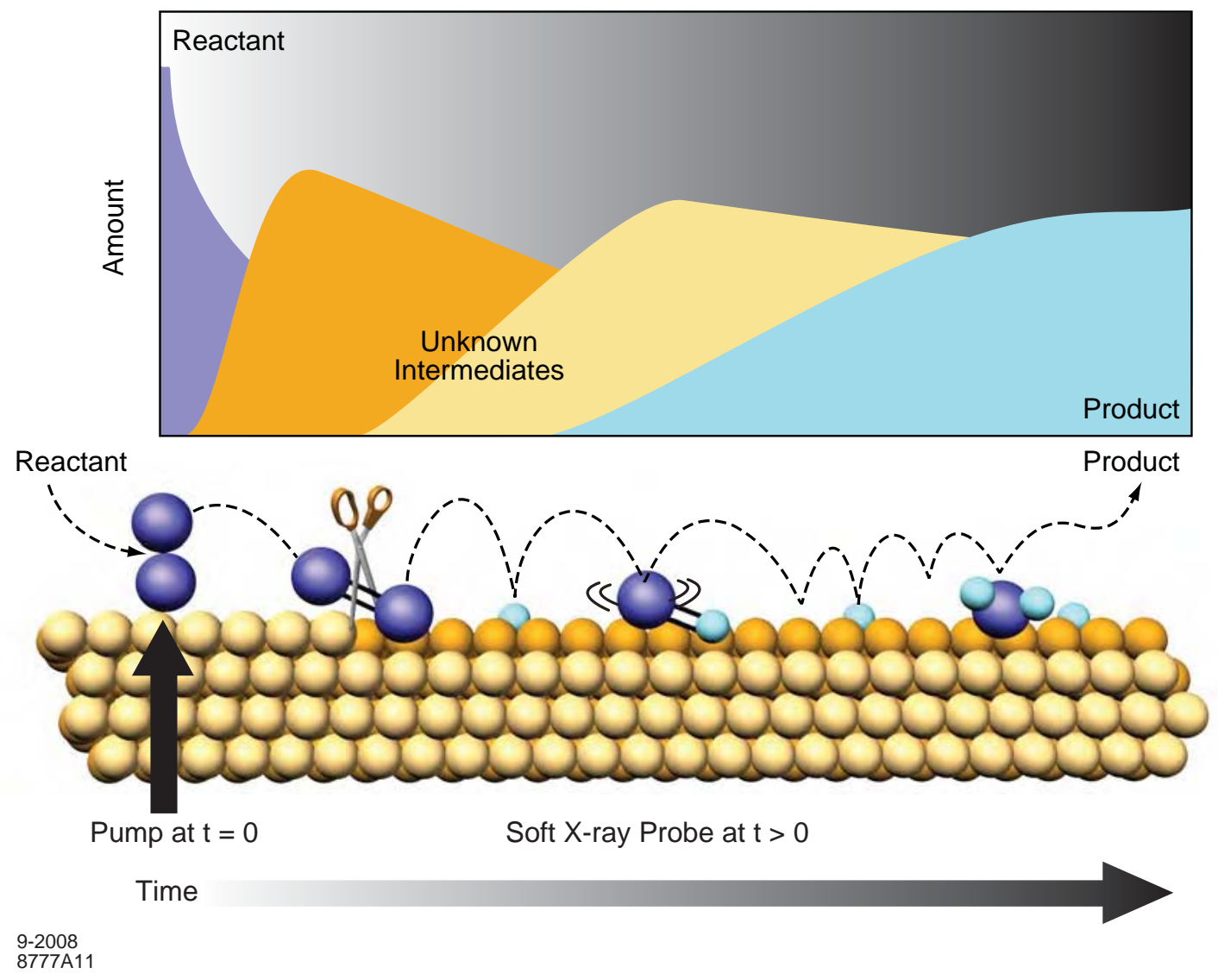

Figure 3.4. Elementary steps in a catalytic reaction involving molecular oxygen and hydrogen. The reaction can be stimulated by a laser of $\mathrm{THz}$ pump pulse and probed with an FEL soft $\mathrm{x}$-ray probe pulse.

In order to optimize catalysts it is essential to develop predictive experimental capabilities of the chemical dynamics. With new x-ray sources we have the potential to experimentally resolve the dynamics of elementary steps and the energy transfer with the substrate during a surface reaction. This can be achieved by coupling a controlled far-infrared or $\mathrm{THz}$ radiation pump with a time resolved $\mathrm{x}$-ray spectroscopy probe (e.g., XES and XPS) that provides element specific information about the local electronic and atomic structure. Hard x-rays permit extension of these studies to realistic chemical reactor conditions. 


\section{Heterogeneous Chemistry of Aerosols-Geoengineering and Health}

A deeper understanding of surface catalysis and condensed-phase reactivity will drive a significant advance in the field of aerosol chemistry where chemical reactions occur on the surface of nano-sized particles, or within liquid droplets dispersed in a gas. Such processes are responsible for cloud formation and rain and are a major factor in determining the solar irradiation of the earth, the integrity of the ozone layer, as well as health of humans, plants, and rivers. In order to understand the processes involved, it is mandatory to chemically analyze the surface of these particles in their atmospheric carrier (lower atmosphere to stratosphere) while subjected to solar radiation and ozone. The most suitable tools for these objectives are x-ray-based spectroscopies (see Figure 3.5) or small angel scattering. When carried out with hard x-rays, these studies can be performed under complex and realistic conditions, such as highpressure, in liquids, etc.

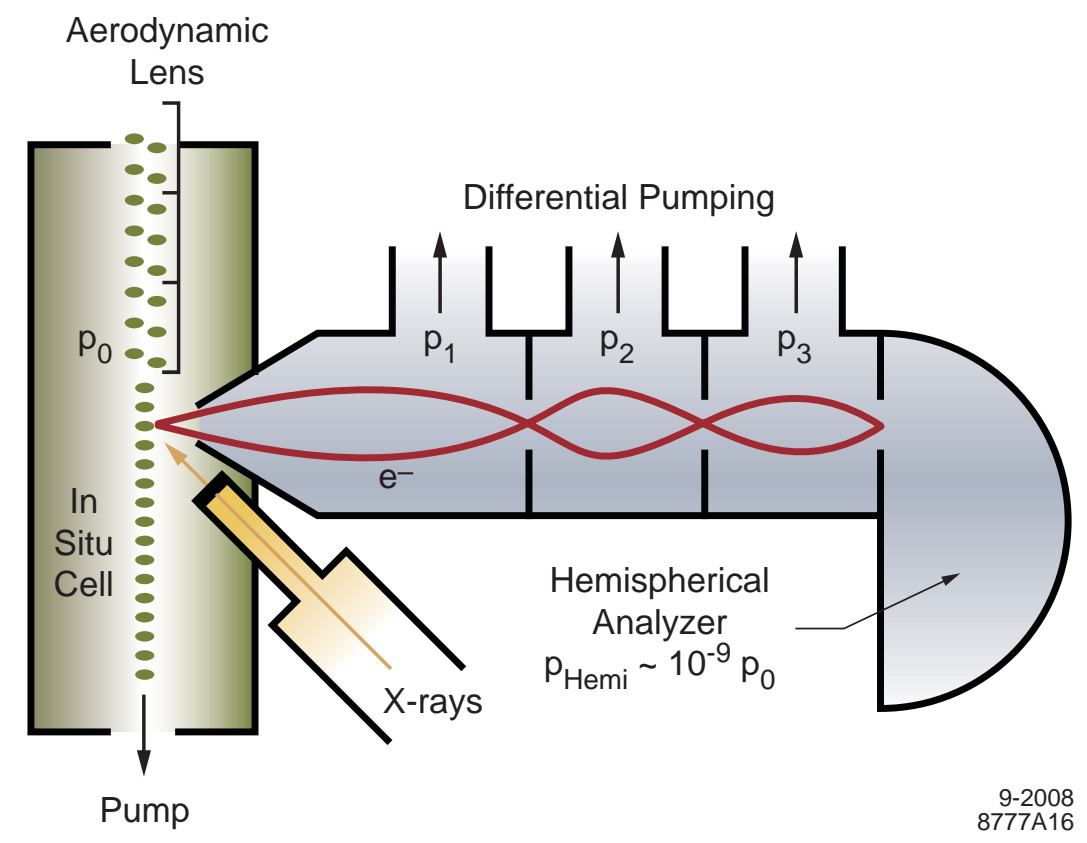

Figure 3.5. Schematics of x-ray spectroscopy setup for in situ aerosol studies. Carrier gas is kept at the appropriate pressure $p_{0}$ while the electron spectrometer is in ultrahigh vacuum.

However, with current $\mathrm{x}$-ray sources such in situ studies are not possible with the relevant particles of $\sim 100 \mathrm{~nm}$ diameter. X-ray sources with a much-increased average brightness are needed for chemical analysis of aerosol surfaces under realistic conditions of aerosol size and concentration $\left(10^{6}-10^{7}\right.$ particles $/ \mathrm{cm}^{3}$ ). In addition, new $\mathrm{x}$-ray sources with ultrashort pulses would make possible time-resolved studies in the femtosecond to picosecond range that could reveal the reactivity of particles in optically excited states.

The impact of such studies would be dramatic. We could use the knowledge for modeling the influence of anthropogenic aerosols on climate and health and for effective rain making and hail prevention through cloud seeding. Furthermore, the risks connected with the controlled injection of nano-sized aerosols into the stratosphere to counteract global warming could be minimized. Such injections are cost effective, but their implementation is presently too risky because of possible side effects, including the depletion of the ozone layer as well as reduced rainfall, soil moisture, and river flow. 


\section{Far from Equilibrium Chemical Processes in a Functional Solar Cell}

Most of the chemical processes in a vast majority of living systems in nature perform their functions when they are driven by conditions that are far from equilibrium. Extreme length (0.1-10 nm) and time scales (fs-ps) are characteristic of this behavior of dissipative systems. However, in the past century our attention has mostly been on the average structural changes in chemical reactions. The focus during the new century is on observing, describing, and controlling the time-evolution of molecular structure during a chemical process. This is an area of fundamental importance, whether it is molecular bonding during a chemical reaction, or the folding of a protein into its physiologically active structure, or the flow of energy in a solar cell or a storage battery.

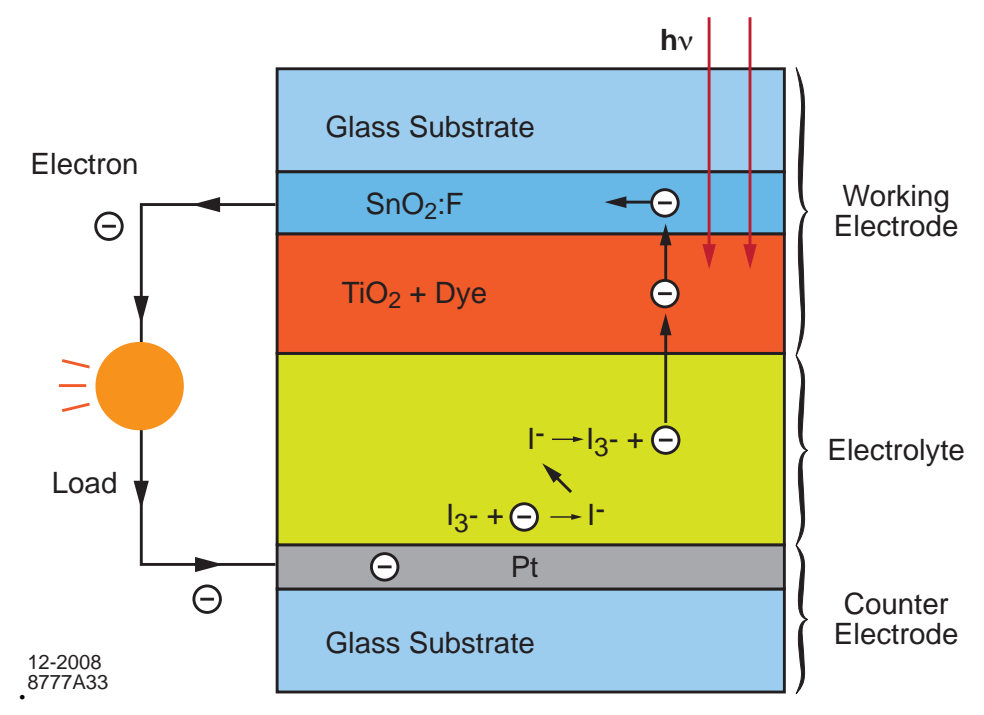

Figure 3.6. Schematic representation of the principle of a dye-sensitized solar cell (DSSC).

[Source: Michael Graetzel, Swiss Federal Institute of Technology, Lausanne]

A technologically important example is the investigation of chemical reaction intermediates is a dyesensitized solar cell (DSSC) shown in Figure 3.6. In the DSSC (also known as Graetzel cell), solar photons are absorbed by the dye molecules. The excited dye injects an electron into the nanocrystalline $\mathrm{TiO}_{2}$ material. In this picosecond to femtosecond process the electrons are transported through the $\mathrm{TiO}_{2}$ to the $\mathrm{SnO}_{2}: \mathrm{F}$ electrode. The dye molecules are left in an oxidized state and reach the ground state through the redox capture of the electrons. Electron migration across the nanocrystalline film may take hundreds of nanoseconds to milliseconds. The dynamical range of redox processes involved in the conversion of light to electric power by DSSC can only be studied in detail by time resolved hard x-ray scattering and spectroscopy techniques. Time-resolved techniques in the fs-ms time domain with few nm spatial resolution using hard x-rays will bring us to a new level of knowledge of chemical reactions in molecular and collective dynamics in solution-phase chemical and biophysical problems. This will enhance our understanding of electron and proton transfer processes, isomerization, photodissociation and structural changes that have broad ranging applicability in energy technologies.

\section{What Is Needed?}

The experiments discussed in this section will make use of various pump-probe configurations. Work on photosynthesis requires visible light excitation and very bright x-ray probes ranging from femto- to 
millisecond time resolution. For spectroscopy experiments that require scanning the $\mathrm{x}$-ray beam energy (e.g., NEXAFS and EXAFS) in microsecond studies a very stable high-average-brightness source is required. For spectroscopy studies based on wavelength-dispersive x-ray optics, either sources with ultrashort $\mathrm{x}$-ray pulses or high average brightness might be best suited, depending on the timescale of interest, sample and sensitivity to radiation damage. Multi-dimensional x-ray spectroscopy to follow both the coherent and incoherent flow of valence charge between different active metal sites will require twocolor $\mathrm{x}$-ray pump-X-ray probe capabilities in the soft and hard $\mathrm{x}$-ray regimes.

In the surface catalysis studies, ultrafast soft and hard x-ray probes will follow the excitation by a short pulse in the THz, visible, or UV. The repetition rate will vary depending on whether systems are reversible or not and at which speed non-reversible systems can be renewed.

Work on aerosols requires very a high average brightness source for chemical analysis and pump pulses in the optical and near UV range combined with ultrashort x-ray probe pulses for reactivity studies.

A future hard x-ray high-rep rate source such as an ERL or FEL beam with a spatial resolution of a few nanometers would penetrate a catalytic reaction cell and provide information on its inner workings of functional components with an unprecedented chemical and structural sensitivity, and femtosecond time resolution.

\section{Understanding Complex Materials}

Central to all the DOE-BES grand challenges is the ability to determine and control electron and spin properties of matter with high precision in the energy, momentum, space, and time domains. "Materials," are a key form of matter that are the subject of core scientific and engineering disciplines and often drive progress in technology. More recently, materials in the form of nanostructures have become of increasing importance. In this section we discuss challenges in understanding the behavior of "complex materials," which exhibit properties that cannot be explained by assuming independent electrons but arise from their correlations. Such materials come in various forms, ranging from the pure elemental ferromagnets with a strong exchange interaction between the electronic spins, to oxide-based "correlated materials" with coupling of charges and spins into nanoscale regions, to multiferroics with a unique coupling of electric and magnetic order parameters, to glassy materials such as colloidal gels and glasses.

Today, a key challenge of condensed-matter physics lies in the understanding and control of such strongly correlated materials on the nanoscale. The intrinsic complexity of such "quantum matter" is illustrated in Figure 3.7. While bulk thermodynamic and transport measurements provide much information about these materials, truly deep insight on the level of atoms, electrons, and spins usually comes from scattering, spectroscopy, and imaging experiments. With the power of future light sources, we can for the first time perform combined scattering-spectroscopy-imaging experiments with sufficient resolution and sensitivity to study important emerging properties, and to study them in real systems.

\section{Electronic Organization on the Nanoscale}

One of the defining characteristics of strongly correlated systems is the presence of nanoscale electronic inhomogeneities. These appear to be self-organized, intrinsic structures that lie at the heart of the all- 
(a)

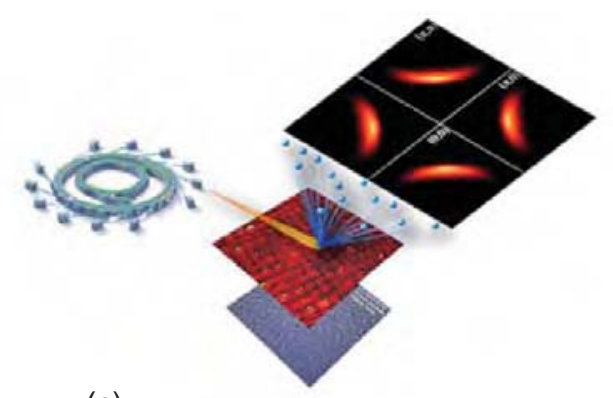

(c)

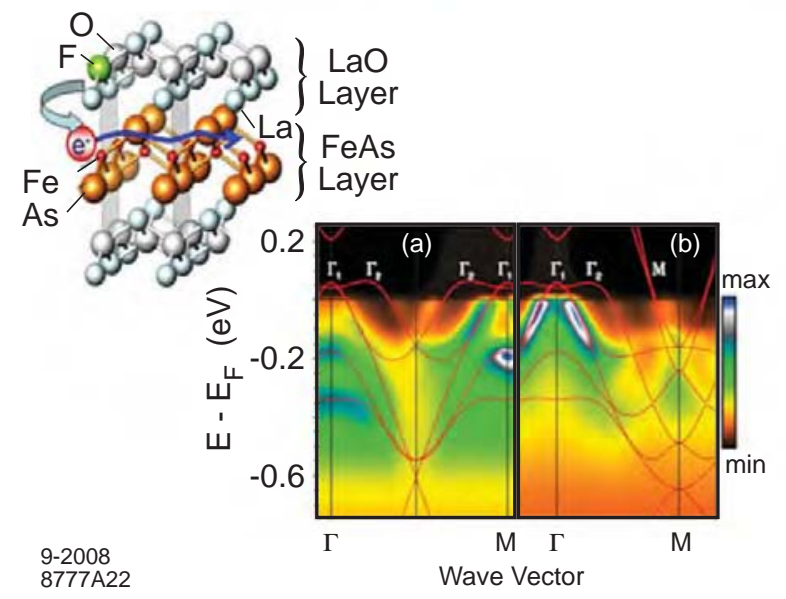

(b)

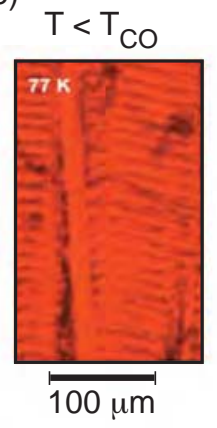

(d)
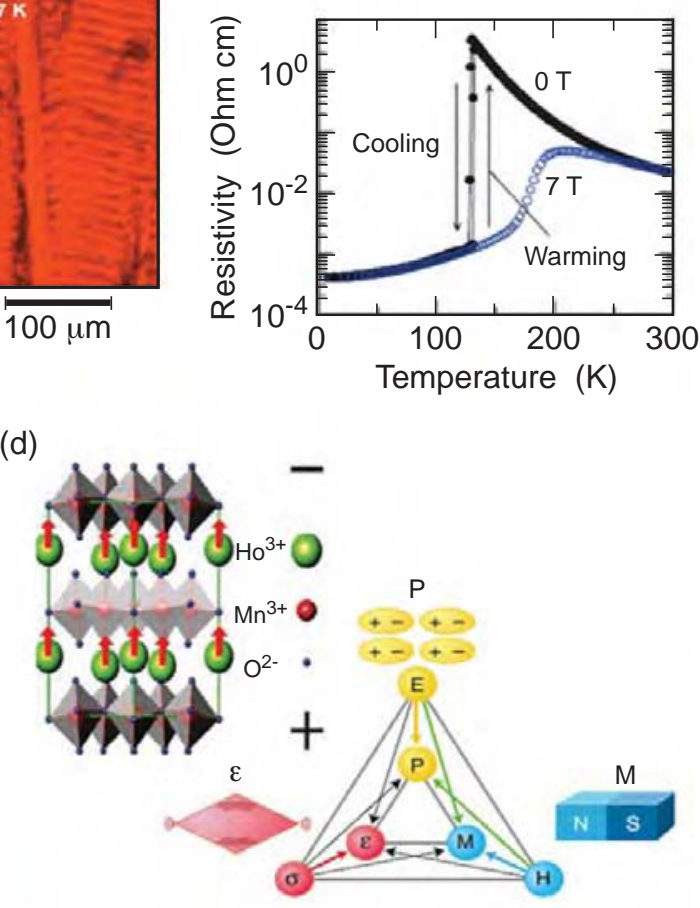

Figure 3.7. Examples of complex quantum materials: (a) High- $T_{c}$ cuprates. (b) Manganites. [Source: $Y$. Tokura and N. Nagaosa, Science 288, 462 (2000)] (c) New Fe-based superconductors. (d) Multiferroics. [Source: N. A. Spaldin and M. Fiebig, Science 309, 391(2005)]

important nonlinear responses of these materials. The ability to probe the electronic organization at these length scales and to produce real-space structural, electronic and strain maps of these nano-domains (for example, organized stripes) will be essential in developing a complete understanding of these materials and is critical to the use of these structures in devices. This will require future ultra-high flux and ultrahigh brightness photon sources.

As an example, a diversity of phase separated domain structures have been reported in manganite multiferroics, with length scales ranging from nanometers to micrometers. This is attributed to the interplay between spin/charge/lattice degrees of freedom along with long-range strain fields. Two and three dimensional imaging of phase-separated domains is possible at third-generation soft and hard x-ray sources using spatially resolved techniques such as XPS, x-ray fluorescence microscopy, and polychromatic diffraction techniques.

Figure 3.8 shows an x-ray fluorescence microscopy image of striped domains of a few micron width together with their detailed crystal orientation obtained from a spatially resolved polychromatic 3-D diffraction image. To go beyond the kind of qualitative understanding that is possible from such images, we will need the ability to study the detailed structure of the interfaces between the stripe domains and the associated strain fields in real space with nm resolution to elucidate the details of the domain walls and their driving physics. While such resolutions should be possible at NSLS-II or a renewed APS for systems 


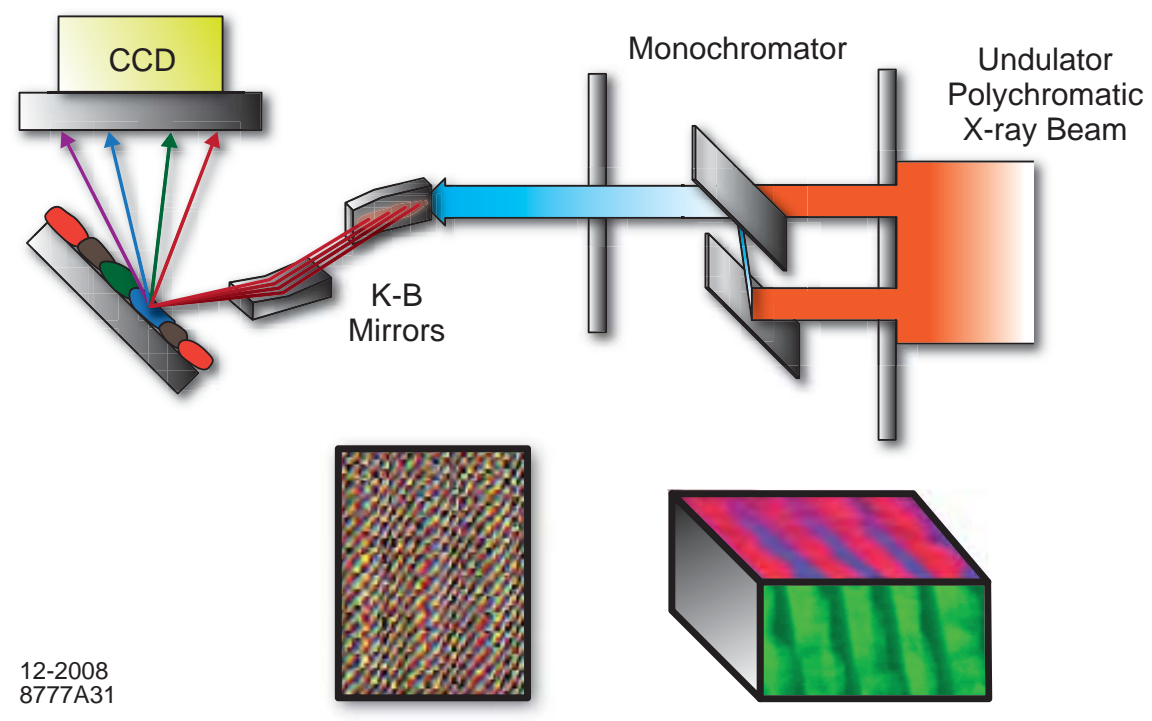

Figure 3.8. Top: Schematic of spatially resolved polychromatic diffraction set up. Typical resolution is about 100 $\mathrm{nm}$ Left: X-ray fluorescent microscopy image of $(\mathrm{Eu}, \mathrm{Y}) \mathrm{MnO}_{3}$ single crystal surface showing ordered alternating stripes with either $Y$ or Eu enrichment phases. Right: the 3-D diffraction image showing alternate lamella of orthorhombic phase of $\mathrm{EuMnO}_{3}$ and hexagonal phase of $\mathrm{YMnO}_{3}$. [Source J. Budai]

with strong scattering contrast - such as the crystallographic domains observed here, what will be required is an ability to probe the electronic organization itself at these resolutions. Examples of this include the charge and spin stripes in cuprates superconductors or in the coexistence of spin density wave/superconducting state in the iron-arsenide superconductors. Scattering from such structures is typically five orders of magnitude or more weaker than crystallographic Bragg peaks and will require substantially brighter sources, beyond storage rings like NSLS-II.

Further, advances in the epitaxial-growth technology for transition-metal oxide thin films and superlattices has led to the discovery of many intriguing nonlinear properties at the heterointerfaces with potential for new applications - for example, in correlated-oxide interfaces with metal electrodes. This promises to be an area of tremendous development in the coming years. Probing the structure and dynamics of the phase separated structures and their interfaces and their response to external stimuli such as the magnetic or electric field or laser pulses is essential in developing sufficient understanding to turn these unique properties into practical devices. Such experiments will require ultra-bright, high-flux photon sources.

\section{Electronic Structure on the Nanoscale}

A second component to understanding these nanoscaled regions will be to probe their electronic structure, that is the dispersion relations of the quasi-particles in these regions. A natural tool is angle-resolved photoemission spectroscopy (ARPES) which has emerged as the mainstream experimental probe of electronic structure and low-lying excitations. Modern high-resolution ARPES provides unprecedented insight into the electronic structure of solids by measuring the single-particle spectral function in energy and momentum, $A(\mathbf{k}, \omega)$. The technique has had major impacts on a number of important fields, such as high-temperature superconductivity, colossal magnetoresistance, and most recently graphene and topological insulators. However, today ARPES today can only provide spatially averaged signals, resulting in the loss of critical information. ARPES with nanometer spatial resolution (nano-ARPES) overcomes this problem, providing information from individual ordered regions, thus combining information in space, 
momentum, and energy. Pioneering instrumentation is being developed at the ALS to measure the electronic properties down to a length scale of $50 \mathrm{~nm}$, limited by the available photon flux density, to understand the electronic states within individual domains as illustrated in Figure 3.9.

(a)

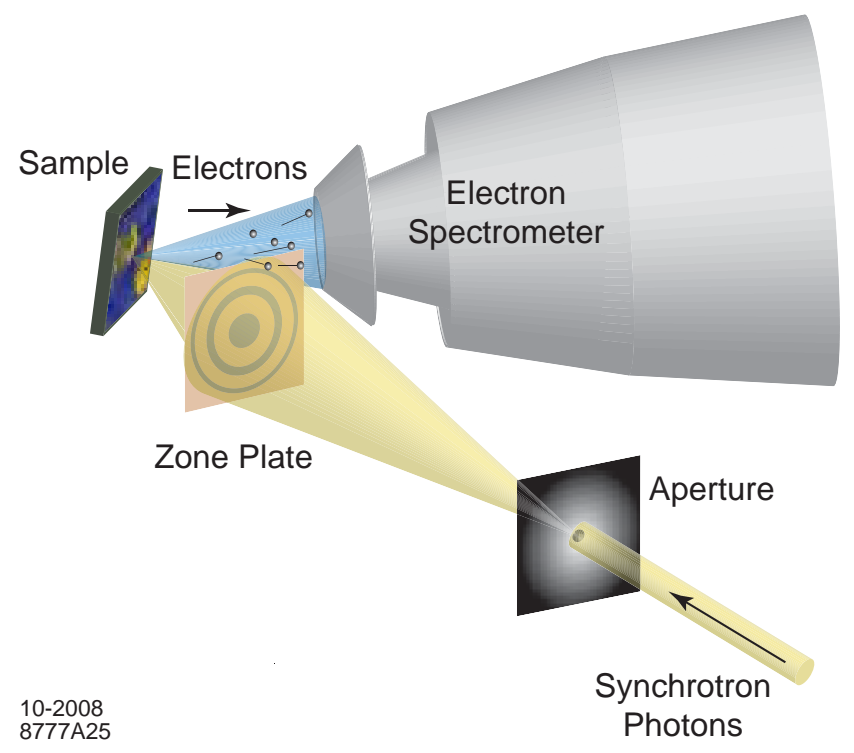

(b)
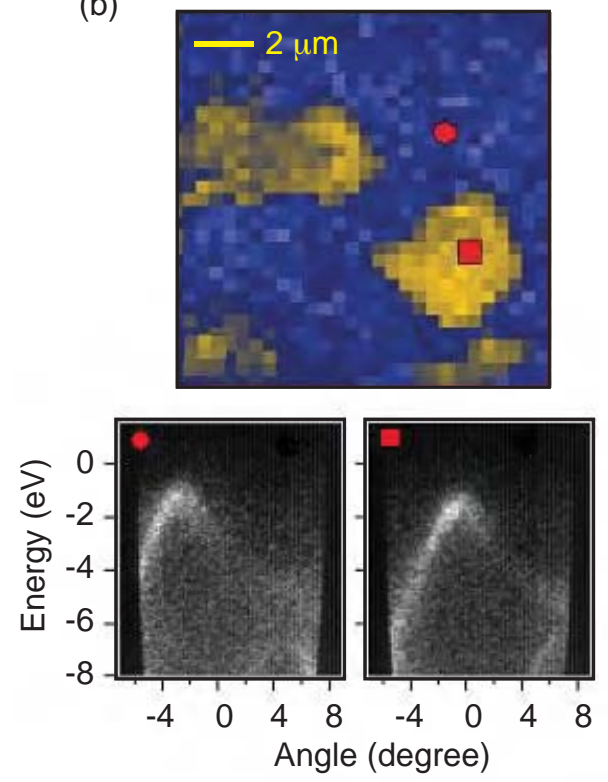

Figure 3.9. (a) Schematic of nano-ARPES set up. (b) Top: nano-ARPES images of randomly oriented crystalline grains of graphite. Bottom: bands acquired from individual crystalline grains. The shift in the bands in the angle direction corresponds to the orientation of each grain. (Conventional spatially integrating ARPES reveals only an average, smeared band). [Source: E. Rothenberg]

Nano-ARPES experiments are best carried out on ring-based sources because space-charge issues require the reduction of the number of photons per pulse, and in rings this reduction may be compensated by high repetition rates. Another breakthrough that will be enabled by future rings is spin-resolved ARPES, which in the past has been impeded by the low efficiency (about $10^{-4}$ ) of spin detectors. This technique is envisioned to become widely applicable with the ability of future sources to go to space charge limited photon flux per unit area. High-energy ARPES is attractive because of increased bulk sensitivity, but has lower cross sections and will benefit greatly from more brilliant sources. The last area where the new sources will make an impact would be time-resolved ARPES, which enables one to directly observe the time evolution of electronic states. Pioneering experiments using lasers indicate exciting promise, but new light sources are needed to fully exploit the potential of time-resolved ARPES for the study of complex materials.

\section{Electron Dynamics of Correlated Materials}

Correlated electron systems display a range of intriguing properties - emergent phenomena - as a result of the strong coupling between the charge, spin, lattice and orbital degrees of freedom. These properties range from high-temperature superconductivity to magnetism to ferroelectricity. Remarkably these ground states frequently occur in the same material or class of materials and appear to be coupled, raising the possibility of new classes of functional materials that utilize one order parameter to control another. Such multifunctional materials would be transformative in fields such as energy generation and transmission 
and information storage and manipulation. The ability to understand, control and utilize these functionalities in next generation materials is therefore one of the major goals of condensed matter physics and materials science in the $21^{\text {st }}$ century.

The functionality of these materials - their response to external perturbations - is encoded in the dynamic response function of the material. This in turn is determined by the excitation spectrum. Of particular importance here are the electronic excitations which dominate the response. The relevant energy scales extend from a few meV up to several $\mathrm{eV}$ and what is required is a momentum-resolved probe of these electronic excitations to fully map out the excitation spectrum. While inelastic neutron scattering has provided a great deal of insight into the spin and lattice excitations in bulk systems, neutrons do not couple to the charge and are unable to study the most relevant excitations. Further, future sample morphologies, driven by technological requirements, will center on thin films, interfaces and nanoscaled systems, and neutron scattering is unlikely to be of direct relevance in such cases.

Inelastic x-ray scattering (IXS) probes the dynamics of condensed matter systems in the energy domain. A photon-in - photon-out technique, it offers a momentum-resolved, bulk-sensitive probe of excitations from $1 \mathrm{meV}$ up to 10 's of eV and with momentum transfers from $0.1 \AA^{-1}$ to $10 \AA^{-1}$. The technique also offers compatibility with insulating samples (organics, bio-materials etc.); external fields (magnetic, electric), pressure, and optical excitation; and sensitivity to symmetry-forbidden optical transitions. However, IXS experiments are very photon hungry, and measurements of electronic excitations with meV resolutions are beyond the capabilities of existing photon sources. At the best synchrotron sources operating today, the flux on the sample is $3 \times 10^{10}$ photons-s $^{-1}$ in a band pass of $6 \mathrm{meV}$. While this is sufficient to measure phonons, which have a relatively large cross-section, it is inadequate for measuring any electronic excitations.

A transform-limited FEL would offer a tremendous gain for this field. Here, the energy band pass is inversely related to the pulse duration. For a $100 \mathrm{fs}$ pulse, this band width would be $6 \mathrm{meV}$. This would be sufficient for many experiments and would eliminate the need for conventional upstream optics. Timeaveraged fluxes on the order of $10^{15}$ photons- $\mathrm{s}^{-1}$ at the sample would then be expected at such sources. This would represent a gain of five orders of magnitude over existing instruments.

Measurements of electronic excitations with resolutions of a few meV at such sources would be gamechanging, as illustrated in Figure 3.10. Important excitations to be studied include electronic pairing gaps (e.g. superconducting gaps and charge-density-wave gaps with typical energy scales of $10 \mathrm{meV}$ ), collective excitations (so-called "orbitons", particle-hole excitations, "holons", etc with energy scales of $\sim 500 \mathrm{meV}$ ) and more exotic excitations (e.g. the pseudogap in high- $\mathrm{T}_{\mathrm{c}}$ and new modes predicted for broken gauge symmetries in high- $\mathrm{T}_{\mathrm{c}}$ 's with energy scales in the 100-1000 meV range). In addition, there are the magnons (20-500 meV) and optical phonons (20-100 meV) and at higher energy scales excitons, charge transfer gaps, interband transitions, and collective plasmon excitations. To take one example, calculated cross-sections for measurements of the superconducting gap in a high- $\mathrm{T}_{\mathrm{c}}$ cuprate translate into count rates of $10^{-3}-10^{-2}$ counts-s $\mathrm{s}^{-1}$ at the best synchrotrons operating today - well below the observable threshold. At a seeded FEL, these same experiments would have count rates of $10^{2}-10^{3}$ counts-s ${ }^{-1}$, and would become feasible for the first time. Similarly, the pseudo-gap in high- $\mathrm{T}_{\mathrm{c}}$ 's remains one of the great mysteries of these materials, with the general belief that it is an electronic continuum of some kind. IXS measurements at an FEL could probe the energy and momentum dependence of this continuum and would reveal a great deal about its nature. 


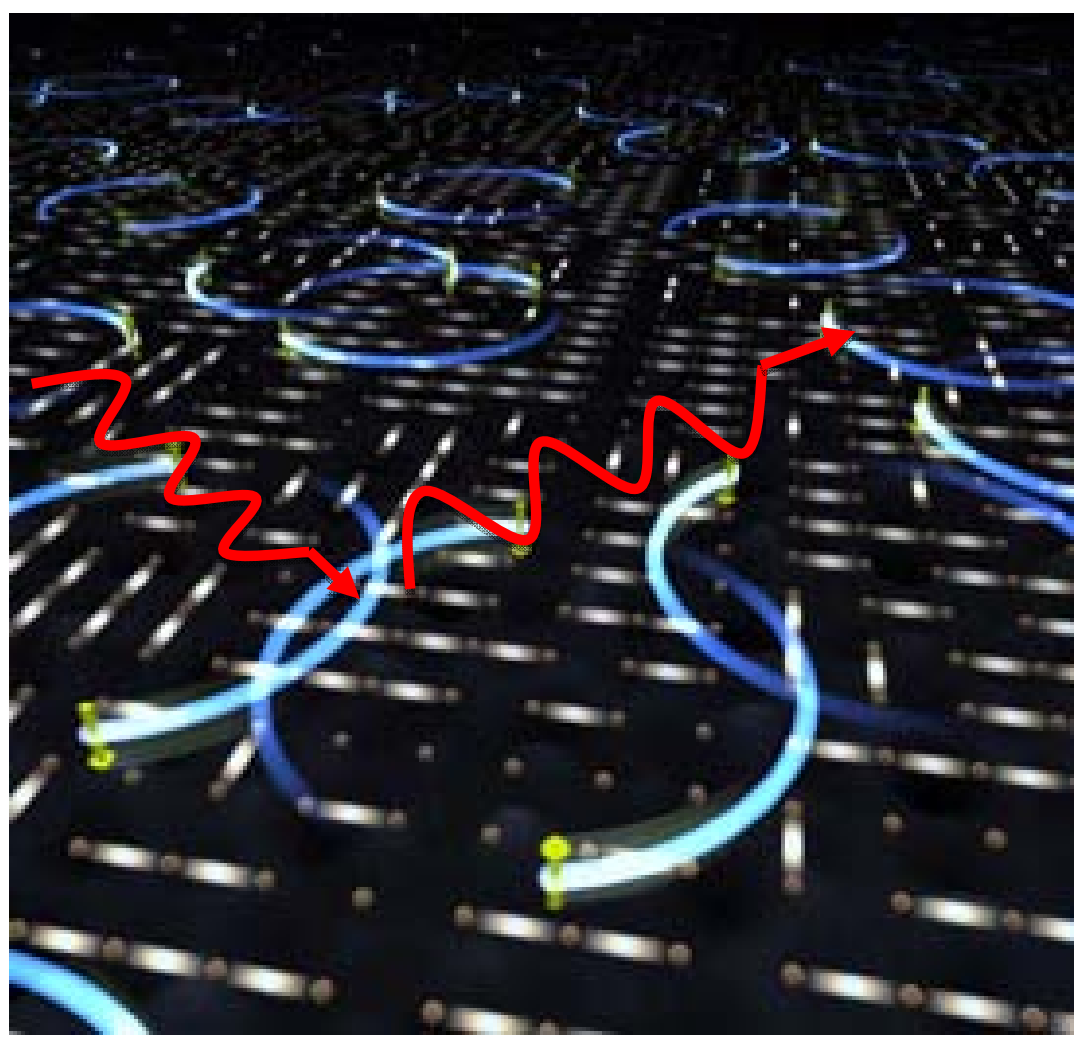

Figure 3.10. (a) Schematic of inelastic x-ray scattering from a Cooper pair in a high-temperature superconductor. The outgoing photon has a longer wavelength than the incident photon and this energy difference is the energy required to break the Cooper pair. Such measurements will be possible at a transform limited FEL for the first time and will reveal the dynamics behind the electron pairing that lie at the heart of hightemperature superconductivity. [Source: J. C. Davis].

Other critical areas to study include non-equilibrium kinetics, possibly interrogating transient intermediates of a system driven by external bias, such as pump probe experiments. An example here would involve exciting carriers across the insulating gap in a high- $\mathrm{T}_{\mathrm{c}}$ (or indeed the superconducting gap) using optical lasers and studying the recombination dynamics of the carriers (or Cooper pairs) as a function of delay time. These studies could be combined with high magnetic fields - possibly pulsed fields that would be synchronized with the FEL pulse - and mK temperatures to allow quantum criticality to be studied. Equally interesting, would be the use of high-transient pressures (e.g. using projectiles or laser shocks). Reaching further, one might imagine coherent Raman scattering or stimulated Raman scattering, which is formally similar to resonant IXS, and is a four-wave mixing process whereby three incident fields $E_{n}\left(k_{n}, \omega_{n}\right) \mid n=1,2,3$, generate a stimulated signal, e.g., $E_{\text {sig }}=-\omega_{1}+\omega_{2}+\omega_{3}$, in the momentum-matched direction, $\mathrm{k}_{\mathrm{sig}}=-\mathrm{k}_{1}+\mathrm{k}_{2}+\mathrm{k}_{3}$ (see for example, Section 3.A.3). This kind of spectroscopy allows one to detect the correlation between two processes in a material system-a new dimension to understand the correlated materials.

\section{$4 \quad$ Nanoscale Dynamics: Equilibrium Fluctuations}

The study of the dynamical properties of nanoscale order in complex materials also provides a severe challenge. The natural time scales of the physical processes in such systems span an enormous range of 
about fifteen orders of magnitude, depending on the atomic, electronic, and spin structure and parameters such as temperature (see Figure 2.1). Observation of materials with nanoscale resolution over this broad time scale can only be effectively covered by a combination of ring-based sources, such as storage rings and ERLs, and FELs.

The natural time scale associated with nanoscale order, which is associated with non-repetitive processes such as equilibrium fluctuations, can be directly studied by x-ray scattering techniques, either in the energy domain by IXS, as discussed above, or in the time domain with coherent X-ray scattering. In both cases the dynamic information represents statistical averages as a function of momentum transfer or length scales in the sample. In this latter technique, any temporal change within the sample region is directly reflected by an intensity modification within the coherent scattering (speckle) pattern. Often it is not even necessary to invert the speckle pattern into real space but simply to record its time dependence as illustrated in Figure 3.11 .
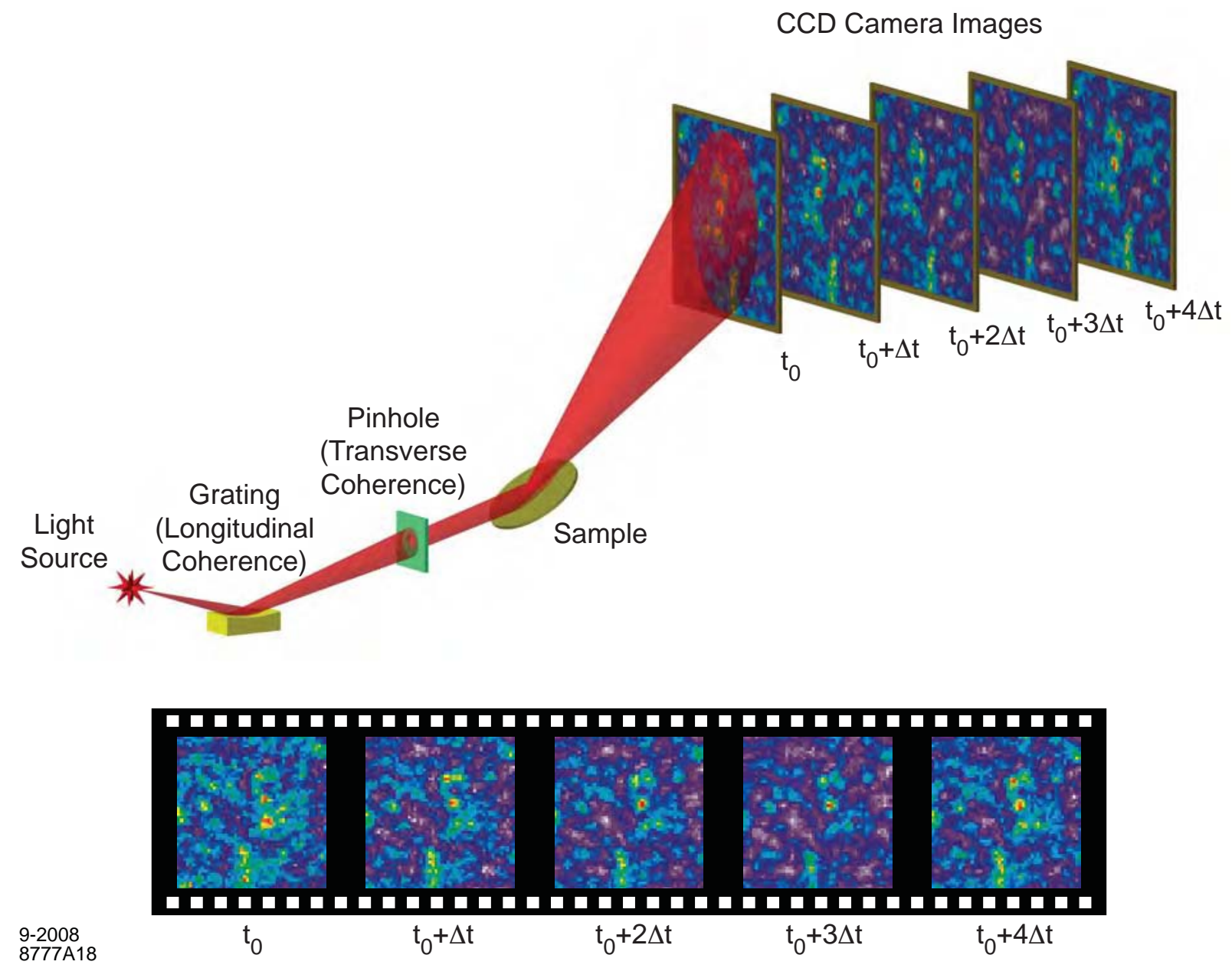

Figure 3.11. Movie of the "speckle" pattern (frames were taken 240 seconds apart and each frame averaged 96 seconds) from the orbitally ordered domains in the cubic manganite $\operatorname{Pr}_{0.5} \mathrm{Ca}_{0.5} \mathrm{MnO}_{3}$, recorded at the ALS. The slow dynamics of the image indicates that the domain walls are pinned by disorder, but the nature of the pinning is unknown. [Source: J. J. Turner et. al., New J. Phys. 10, 053023 (2008)]

The results suggest that physics on the large "mesoscopic" length scale is intimately related to atomic and/or electronic disorder at the angstrom scale. Naturally, one would like to extend such experiments, 
presently limited to time scales of many seconds, to much shorter time-scales. The signal to noise ratio of such experiments scales as $\tau^{1 / 2} B \sigma$ with the sampling time $\tau$, the source brightness $B$, and the scattering cross-section per volume $\sigma$ and thus will greatly benefit from the increase in average brightness available on future rings. At the ALS today, dynamics of nanoscaled orbital domains can be probed on a time scale of 10's of seconds. At NSLS-II, this will be pushed to 10's of ms. To push into the microseconds and faster, that is time scales relevant for functional devices, will require ultra-bright, high-repetition rate sources. Further, while soft $\mathrm{x}$-rays (as used in Figure 3.11) are ideal for probing orbital and magnetic fluctuations, charge fluctuations typically occur at larger momentum transfers and will require hard $\mathrm{x}$-rays to access.

As IXS in the energy domain yields information on local collective charge excitations, studies in the time domain offer complementary insight into how interactions on the local level manifest themselves in the formation and dynamics of nanoscale domains. This is one of the promising pathways for using $\mathrm{x}$-rays to open windows into the phenomenon of emergence, linking cooperative charge dynamics from both local and nanoscale perspectives.

Faster dynamics require the use of x-ray FELs (X-FELs) and fast 2D detectors with time correlators, so that the speckle patterns recorded with individual ultrafast pulses may be compared. Such comparison depends on the readout speed of position-sensitive detectors, which is presently inadequate and needs to be greatly improved. In the near future, this limitation can be overcome by comparing images recorded with one shot with those recorded with multiple shots, so that temporal changes are reflected by increased blurring with the number of shots. With suitable delay lines it will be possible to study nanoscale dynamics down to the femtosecond time scale.

In principle, real-time motion pictures of nanoscale equilibrium dynamics are also possible by $\mathrm{x}$-ray microscopy, e.g., PEEM, but they are presently also limited to timescales of seconds by the available average $\mathrm{x}$-ray flux density on storage rings. Ultrafast $\mathrm{x}$-ray movies of real-space fluctuations could be produced with adequately spaced X-FEL pulses, each sufficient to record a picture. This, however, also requires the development of ultrafast position sensitive detectors.

\section{$5 \quad$ Nanoscale Dynamics: Driven Phenomena}

In Section 2, the technological bottleneck that exists around 100 ps was discussed. An example of this is the speed limit of writing and reading electronic "bits" in data storage. Since technology depends on repeatable processes their exploration naturally lends itself to pump-probe techniques. Since optical pulses can be ultrafast, one of the forefront questions in magnetism research today concerns the possibility of manipulating the magnetization optically. Figure 3.12a shows that this is indeed possible. The laser pumpprobe experiment shown reveals the ultrafast demagnetization of a ferromagnet by an intense ( $\left.>1 \mathrm{~mJ} / \mathrm{cm}^{2}\right)$ laser "pump" pulse. Figure 3.12b illustrates that the use of circularly polarized light may indeed supply the angular momentum to enable "all-optical switching" on the femtosecond time scale.

While we have now observed such "effects," the underlying processes remain the subject of great controversy. It is clear that the sample evolves through a laser-excited electronic state where the modifications of the magnetization take place. Simply replacing the femtosecond laser probe pulse with ultrashort $\mathrm{x}$-ray probe pulses supplied by a soft x-ray FEL would allow the understanding of this transition state. Time-dependent XMCD measurements would reveal the separate evolution of the spin and orbital 
(a)

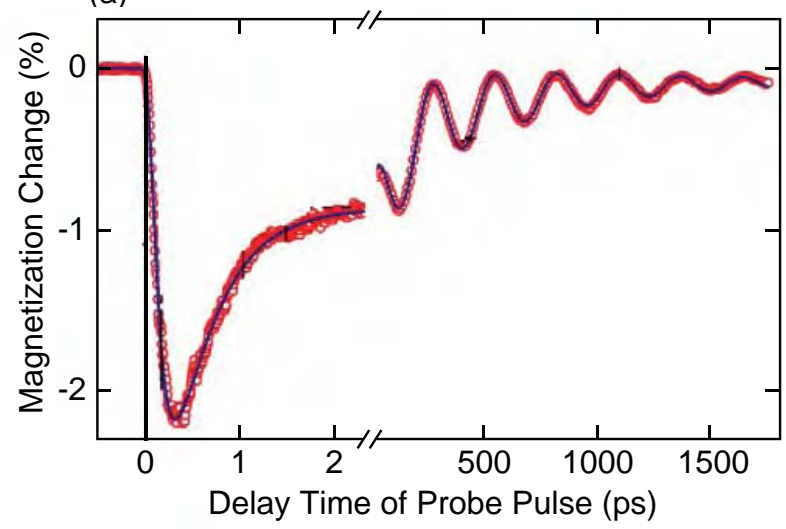

(b)

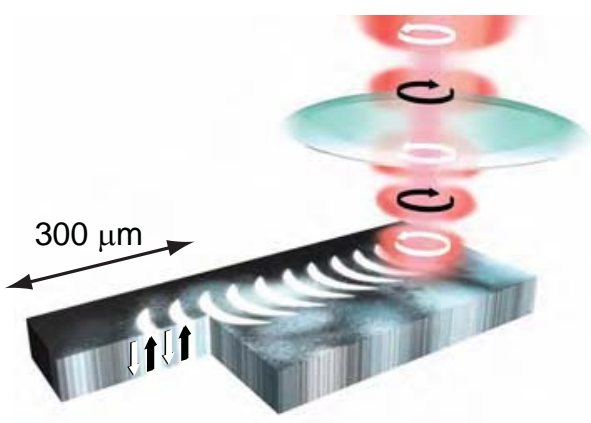

(c)
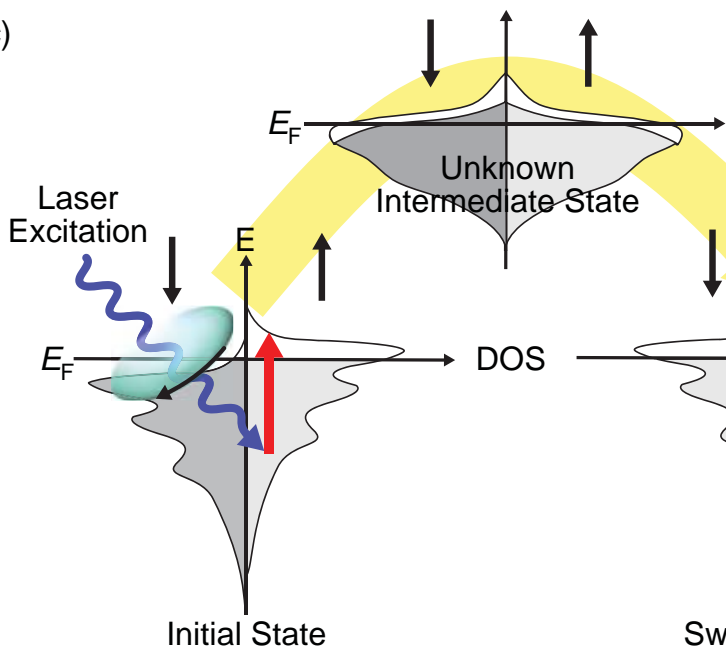

State

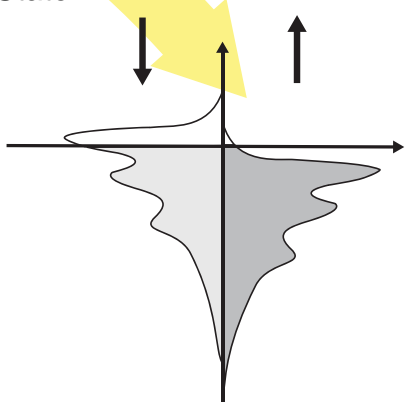

Switched State

$9-2008$
$8777 A 4$

Figure 3.12. (a) Reduction of the perpendicular component of the magnetization $\boldsymbol{M}$ after laser heating a Ni thin film at $t=0$. The sub-picosecond quenching of $M$ is followed by a recovery via electron-phonon relaxation, and at $t>100 \mathrm{ps}$ by a damped precession of $\boldsymbol{M}$ about the applied field. [Source: B. Koopmans et al., Phys. Rev. Lett. 95, 267207 (2005)] (b) Demonstration of compact all-optical recording of magnetic bits in $\mathrm{Gd}_{22} \mathrm{Fe}_{74.6} \mathrm{Co}_{3.4}$ by scanning a circularly polarized laser beam across the sample and modulating the polarization of the beam between left and right circular. [Source: C.D. Stanciu et al. Phys. Rev. Lett. 99, 047601 (2007)] (c) The evolution of the spin-dependent electronic structure through excited states after laser excitation remains an unsolved science problem.

components of the magnetization, providing key information on the transfer of angular momentum out of the spin system that has to accompany any magnetization change. Time and spin-resolved photoemission spectra would directly measure changes in the spin-resolved band structure in the laser-excited state. As illustrated in Figure 3.12c, we do not know at present whether and how the electronic bands, the exchange splitting, and the spin-orbit coupling change in the laser-excited state.

\section{$6 \quad$ What Is Needed?}

From the above discussion, it is apparent that complementary access to the nanoscale is provided by both soft and hard x-rays, and therefore bright x-rays over the spectral range from tens of eV to tens of keV are needed.

In some cases, we clearly need the ultrafast capabilities of FEL sources. Often the availability of ultrashort pulses at high repetition rates on the order $100 \mathrm{kHz}$ is necessary to obtain sufficient signal. We envision 
that for certain experiments we may need to reduce the number of photons per pulse to avoid sample "damage" or space-charge effects. An example is the ultrafast spin polarized photoemission experiment discussed in conjunction with Figure 3.12. In other cases, long pulses and control over the longitudinal coherence will be essential - the inelastic x-ray scattering measurements of electronic excitations discussed above, being a prime example.

Further, we envision that even in the future a large number of x-ray users studying "materials" will benefit from high average brightness provided by high rep-rate storage rings or ERLs that also offer superb beam stability in position and time, which is required for optimum signal to noise and background ratios. In practice, the key parameter is often photon flux (number of photons/time/area), not brightness, since techniques such as $\mathrm{x}$-ray absorption, $\mathrm{x}$-ray emission and photoemission are insensitive to momentum spread (angular divergence) of the incident photons and also do not require lateral coherence. In some cases, such as nano-ARPES, high repetition rates are needed to avoid space charge effects while still achieving the desired resolution and signal to noise ratio. This requirement is also obvious for diffraction tools that require large flux focused in nanometer spots and even $4 \pi$ detectors with energy resolution of a few $\mathrm{eV}$ bandwidth at tens of $\mathrm{keV}$ to measure evolving domain structure following the application of external control stimuli.

The complicated problem of the interaction of increasingly intense $\mathrm{x}$-ray pulses with the sample, for both FELs and future rings, will be addressed in more detail in Appendix C.

\section{Novel Materials by Design and Materials Behavior under Extreme Conditions}

The tools of nanoscience to assemble matter by manipulating atoms and molecules have provided new revolutionary synthesis paths. Physical understanding of the assembly process to create a new generation of advanced materials and hierarchically organized systems is of critical interest. In order to harness atomic and molecular manipulation as a tool for bottoms up fabrication of systems with tailored properties we need to establish the thermodynamic and kinetic rules of assembly, and relate it to their functions based on structural, electronic, spin and phonon properties. The dynamics of coherent correlations including stochastic events or fluctuations in properties such as chemical, optical, magnetic, conductivity, or superconductivity in small assemblies of atoms and molecules as they are formed is a focal area.

One recurring thought in the above discussion concerns the discovery of new phases of matter through atomic manipulation. These manipulations can often bring the matter close to instabilities leading to a new phase with unique electronic, spin and structural properties. This is often referred to as emergent phenomena. Their origin is deeply rooted in the complex electronic and vibrational correlations that manifest from unique structural arrangements. For example a mysterious pseudo-gap phase of matter may develop in a composition of complex oxides that is sandwiched between the insulating and superconducting phase. In this emergent phase the electrons appear to develop some of the correlations of a superconductor, for example, without developing full blown superconductivity and form a 'checker board' modulation of electron distribution. How can a monolayer of oxide between insulating blocks exhibit conducting property of a quasi-two-dimensional electron gas? What is the smallest structural unit that exhibits cooperative correlation such as ferroelectric polarization? The tools for these investigations require in situ techniques along with the most powerful structural tools of x-ray spectroscopy and diffraction with unprecedented sensitivity. 
Another approach for synthesizing novel materials uses extreme environments (pressure, temperature, electromagnetic fields, magnetic flux compression, radiation). This may seem like an exotic approach, but nature already provides us with an excellent example, diamond, whose stability field is at high pressure and temperature $(P-T)$, but whose metastability field extends back to ambient conditions. Understanding the high-pressure phase of carbon led to the development of synthesis techniques that mimic its creation (high $P-T$ methods) and that now drive the billion-dollar diamond-abrasives industry. In addition, knowledge of the $\mathrm{sp}^{3}$ carbon bonding of diamond has led to a new synthesis pathway that uses $\mathrm{sp}^{3}$ bonded carbon in a methane plasma as a starting material for chemical-vapor-deposition growth of diamond in a near-vacuum environment; high pressure is no longer required. This synthesis technique shows promise for growing large single-crystal diamonds for high-tech applications.

Pressure drastically alters material structure and properties, thus opening new frontiers in fundamental physics and chemistry, as well as applications to planetary, Earth, biological, and materials sciences. We need to elevate high pressure research to the next level with an integrated, comprehensive approach that combines high pressure with structural, electronic, magnetic, and phonon probes. The resulting combination will enable us to tackle a range of grand challenges including new rules of chemical affinity, reactivity, bonding, and crystal and amorphous structure will be established across the Periodic Table in each pressure regime. Breakthroughs are anticipated in mineralogy, geophysics, geochemistry, bioscience as well as hydrogen storage, stockpile stewardship, and super-hard materials.

The general objective is to interrogate, direct, and manipulate bulk and nanoscale material properties at the level of atoms or electrons in order to discover new synthesis pathways, create new forms of tailored matter, and visualize the fundamental processes underlying materials at their limits. Only a few tools exist to carry out this task. Intense ultrafast electromagnetic fields (from terahertz to x rays) can be used to induce exotic transient phases of matter that may subsequently be quenched into technologically useful metastable states. Magnetic-flux compression creates magnetic fields as large as $1000 \mathrm{~T}$ for a duration of milliseconds, matching naturally occurring exchange fields. Diamond-anvil cells create huge static pressures on materials up to several hundred GPa, as present, for example, in the center of the earth.

Extreme conditions of pressure, temperature and EM fields are also an important tool for understanding the nature of complex materials. The rich variety of phenomena observed for the transition metal oxides (superconductivity, colossal magnetoresistance, multiferroic) reflects the underlying competition between various types of spin, charge and orbital order having similar energy scales. One of the challenges in understanding these materials is to distinguish between these competing orders. The standard approach is to explore the equilibrium phase diagram with the expectation that the various interactions are affected differently according to which thermodynamic property (e.g. applied magnetic or electric field) is varied. A strong transient electric or magnetic field expands the phase diagram to include adjacent nonequilibrium states, and when combined with an ultra-fast x-ray probe, allows one to follow the dynamics for restoring equilibrium.

\section{$1 \quad$ Tailoring Materials with Emergent Properties}

With newly developed nanofabrication facilities, we are now poised to develop a new generation of advanced materials and hierarchically organized systems with tailored structural, electronic, spin and phonon properties. The dynamics of coherent correlations including stochastic events or fluctuations in properties such as chemical, optical, magnetic, conductivity, or superconductivity in small assemblies of atoms and molecules as they are formed is a focal area. The chemical dynamics of melting, dissolution, the 
dynamics of nanocluster formation, the dynamics of surfaces and interfaces are all focal areas. The new properties are often referred to as emergent phenomena, where their origin is deeply rooted in the complex electronic and vibrational correlations that develop with unique structural arrangements.

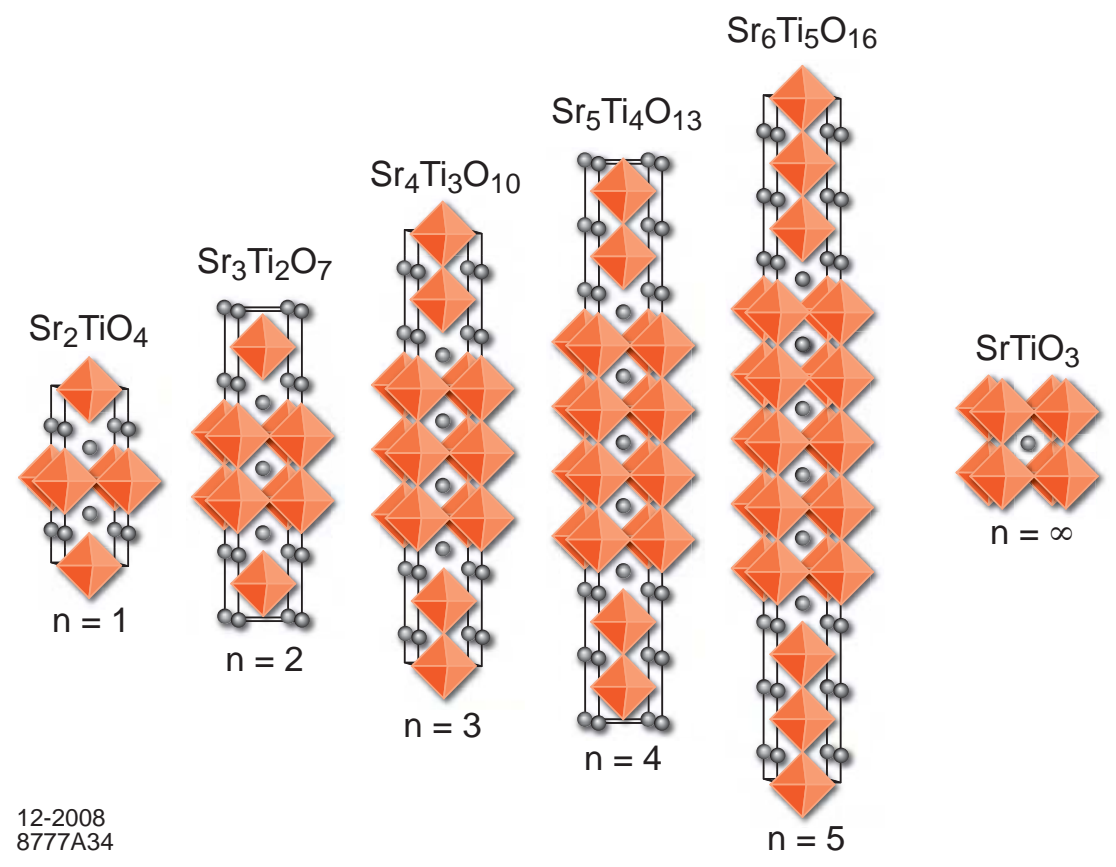

Figure 3.13. Schematic of the crystal structure of a unit cell of first five members of the $\mathrm{Sr}_{n+1} \mathrm{Ti}_{n} \mathrm{O}_{3 n+1}$ Ruddlesden-Popper homologous series, i.e., $\mathrm{Sr}_{2} \mathrm{TiO}_{4}, \mathrm{Sr}_{3} \mathrm{Ti}_{2} \mathrm{O}_{7}, \mathrm{Sr}_{4} \mathrm{Ti}_{3} \mathrm{O}_{10}, \mathrm{Sr}_{5} \mathrm{Ti}_{4} \mathrm{O}_{13}$, and $\mathrm{Sr}_{6} \mathrm{Ti}_{5} \mathrm{O}_{16}$. Circles represent $\mathrm{Sr}$ atoms, while $\mathrm{Ti}$ atoms are at the center of the octahedra (coordination polyhedral) with oxygen atoms at each apex. The inset shows the deposition cycles used in the MBE layering. [Credit: J. H. Haeni et al., Appl. Phys. Lett. 78, 3292 (2001)]

One example, exhibiting 2D growth, is shown in Figure 3.13. The first five members of the $\mathrm{Sr}_{n+1} \mathrm{Ti}_{n} \mathrm{O}_{3 \mathrm{n}+1}$ Ruddlesden-Popper homologous series, i.e., $\mathrm{Sr}_{2} \mathrm{TiO}_{4}, \mathrm{Sr}_{3} \mathrm{Ti}_{2} \mathrm{O}_{7}, \mathrm{Sr}_{4} \mathrm{Ti}_{3} \mathrm{O}_{10}, \mathrm{Sr}_{5} \mathrm{Ti}_{4} \mathrm{O}_{13}$, and $\mathrm{Sr}_{6} \mathrm{Ti}_{5} \mathrm{O}_{16}$, were grown by reactive molecular beam epitaxy and their structural arrangements were monitored by $\mathrm{x}$-ray diffraction. Thin films of this series show a wide variation in emergent dielectric properties, making this approach suitable to tailor materials for various applications. Future systems will demand that the MBE instrument and ancillary techniques be made the part of the x-ray beamline.

The emergence of new properties is not limited to oxides. Many zero-D, 1-D and 2-D nanostructures of semiconducting III-V compounds can have far-reaching emerging properties suitable for many energy applications. Will we someday be able to design and self-assemble zero-D and 1-D nanostructures with specified or emergent properties? Present day soft and hard x-ray sources are being used to evaluate how coherent $\mathrm{x}$-ray diffraction imaging (CXDI) techniques can directly image surfaces, interfaces, and extended defects in quantum systems. Combining multiscale techniques with hard x-ray CXDI in the grazing incidence geometry will add a new dimension to understanding quantum systems. For example, the effect of strain and defects on polarization fatigue within a single a ferroelectic oxide nanodot can be investigated. Performing PEEM to explore magnetic domains and correlating them with CXDI surface structure on a single magnetic nanodot is another challenge. In the future, we can look forward to extending CXDI to include hard x-ray holography using a planar or curved reference wave. 
Beyond quantum systems, nanofoam is a system with smallest pores with unusual mechanical strength making them suitable with application to geology, surfactants, phospholipids, cells, bone structure, polymers, medicine, and structural materials. Materials synthesized with a specific nanopore size distribution, shape and volume of the pore space directly determines their ability to perform a desired function in a specific application. Will we be able to fabricate nanofoam membranes with uniform pore sizes to allow molecules to pass through based on their molecular size, chemical affinity, of ionizing ability? Can we control the flow of DNA and RNA proteins through pores with electrostatic force? Will we be able to fill the pores with excitonic materials for enhanced luminescent properties lighting or LED displays? While we have had great success in understanding microporous materials in the past at the third generation x-ray sources through x-ray tomography, extending this capability to the 3D imaging of nanofoam requires the use of coherent $\mathrm{x}$-rays.

Recently, the labyrinthine internal structure of tantalum oxide nanofoam has been imaged (Figure 3.14) using coherent diffractive imaging (CDI). Controlled tailoring of such structures with predictable functionalities requires spatiotemporal imaging with hard-x-ray sources that can be used during real time processing of the structures.

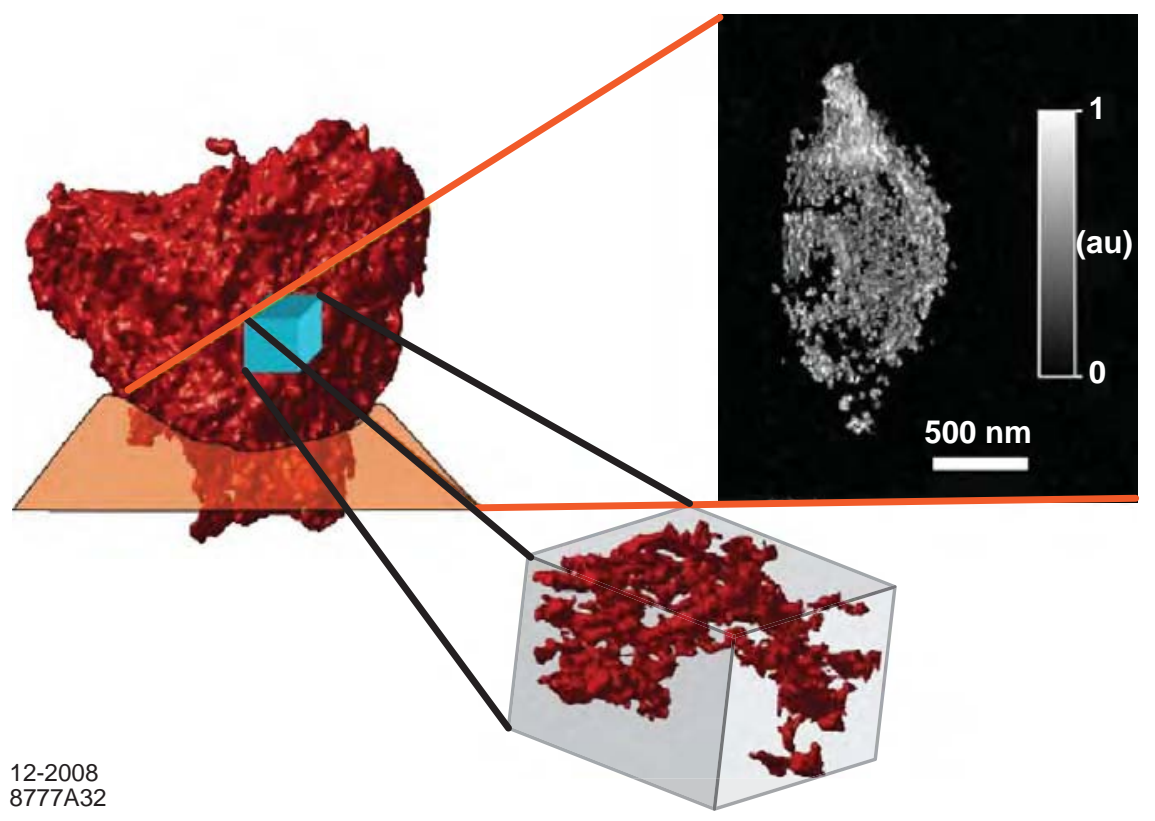

Figure 3.14. Section and isosurface rendering of a $500 \mathrm{~nm}$ cube from the interior of the 3D volume. The foam structure shows globular nodes that are interconnected by thin beamlike struts. Approximately $85 \%$ of the total mass is associated with the nodes, and there is no evidence of a significant fraction of dangling fragments. [Source: A. Barty et al., Phys. Rev. Lett. 101, 0155501 (2008)]

\section{$2 \quad$ Static and Dynamic Compression}

Extreme pressures and temperatures are a still largely unexplored in the search for phases with new properties and the study of the interiors of large planets. They can also point the way to the development of unique new materials that can be synthesized and used at practical conditions. Of critical importance for 
tapping this potential is the ability to characterize the samples and understand their behavior at extreme conditions. X-ray Raman spectroscopy (XRS, see Figure 3.15) is a powerful probe for studying bonding changes in elements and compounds.

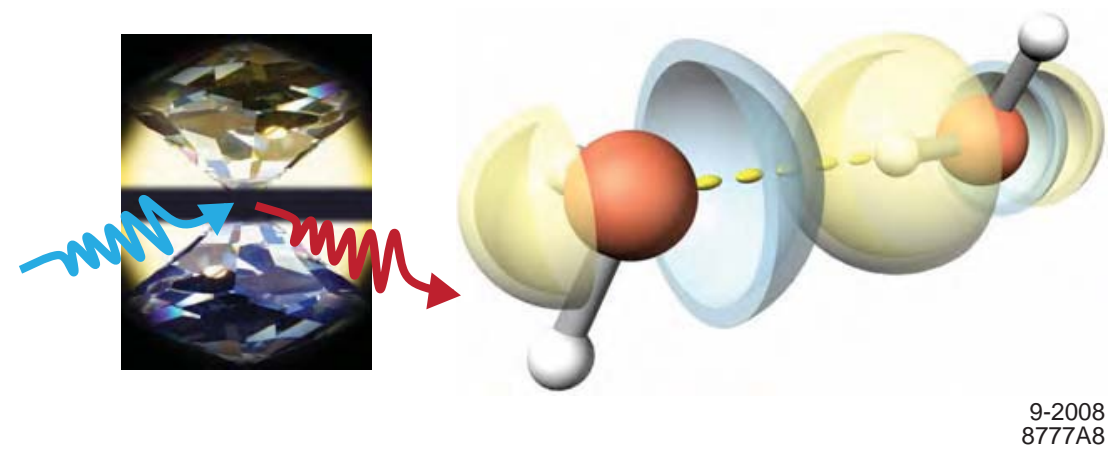

Figure 3.15. In x-ray Raman spectroscopy, hard x-rays are inelastically scattered from a sample inside diamond cell (left). The energy loss excites a core electron to probe empty valence orbitals (right) that are sensitive to the local structure.

This technique has been successfully applied at moderate pressures, but these studies are severely limited with existing x-ray sources, and systematic studies at ultrahigh pressures (> $100 \mathrm{GPa}$ ) are currently not feasible. A much brighter hard x-ray source would overcome the present trade-offs and limitations of sample size, data-acquisition time and accuracy, as well as spatial resolution. It makes possible accurate studies at ultrahigh pressures, simultaneous high temperatures, and temporal resolution that are crucial for next-generation explorations. For example, we would like to be able to study the novel photochemistry and behavior of systems relevant to the production and storage of energy. Very recently it has been found that, at moderate pressure, $\mathrm{H}_{2} \mathrm{O}$ decomposes in the presence of an intense x-ray beam to form a new molecular $\mathrm{H}_{2}-\mathrm{O}_{2}$ compound (Figure 3.16).

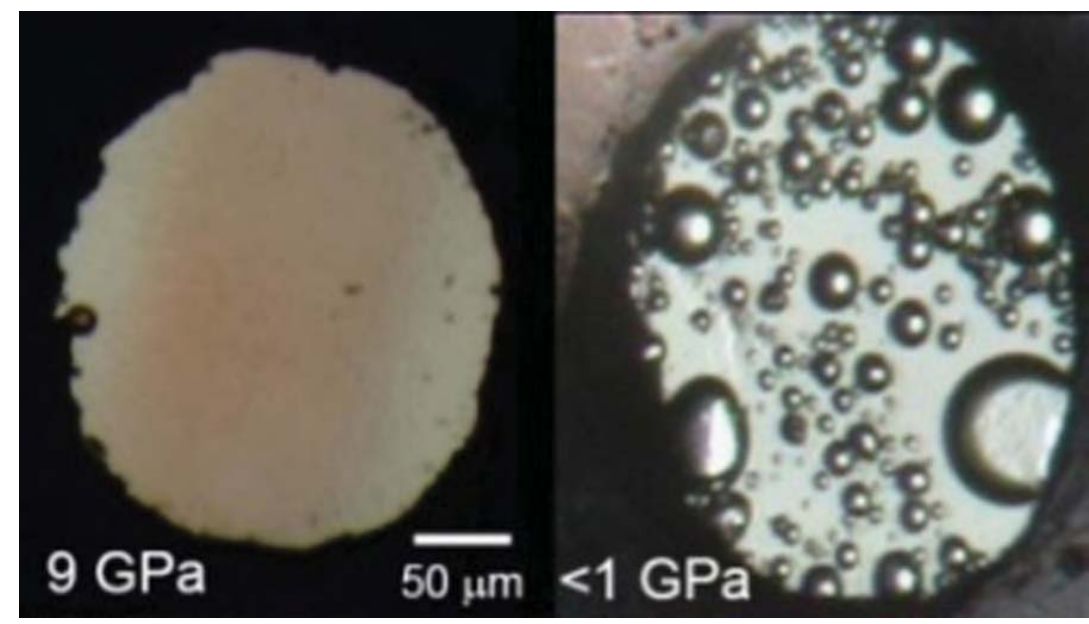

$9-2008$

Figure 3.16. Compressed $\mathrm{H}_{2} \mathrm{O}$ sample after x-ray exposure. Left: $\mathrm{New} \mathrm{H}_{2}-\mathrm{O}_{2}$ compound has formed (see brown region). Right: after decompression, $\mathrm{H}_{2}$ and $\mathrm{O}_{2}$ gases are released. [Source: W. Mao] 
This reaction has implications for understanding how to efficiently split $\mathrm{H}_{2} \mathrm{O}$ and store $\mathrm{H}_{2}$ in a crystalline material. There are many basic questions that need to be addressed in order to understand the reaction process and the new materials that are created. What intermediate species are formed during the reaction? What energy levels are being excited? What metastable states can be created? Are similar phenomena at play in other basic hydrogen-rich molecules like $\mathrm{NH}_{3}$ and $\mathrm{CH}_{4}$ ? Currently, such studies are impractical for following the reaction progress because of the lack of brightness from existing sources. With a much more intense source, we will be led to new synthesis pathways for generating and storage of high-energy-density materials and for understanding fundamental physics and chemistry in simple hydrogen-rich systems.

We need to address the synthesis of metallic hydrogen by directly probing the band gap and the plasmon when $\mathrm{H}_{2}$ metallizes. We could test theoretical predictions for two coexisting superconducting superfluids (electronic and protonic) at the minimum melting point and liquid ground state of hydrogen. Moreover, we could unravel the high $P$-T behavior of $\mathrm{H}_{2} \mathrm{O}$ including the superionic phase, symmetrization of $\mathrm{H}$-bonding, and liquid-liquid transitions through XRS at the oxygen $\mathrm{K}$ edge. With superior spatial resolution, we would be able to carry out XRS studies of chemical bonding of carbon at pressures exceeding $100 \mathrm{GPa}$. Results may reveal the fundamental changes of carbon at high pressures and novel discoveries in inorganic carbon (e.g., $\mathrm{CO}_{2}$ ), organic carbon, and elemental carbon (e.g., fullerene, graphene, and amorphous carbon).

There is a whole new world of condensed state of matter waiting to be discovered with new and emerging behavior that cannot be produced with the compression pressures generated today by diamond anvil cells (DAC). However, dynamic compression can produce compression to reach the new phases of condensed matter shown in Figure 3.17. High-pressure high-temperature studies using DAC have at the thirdgeneration $\mathrm{x}$-ray synchrotron sources have not only added much to our knowledge on unusual equilibrium phases and properties of matter, but also prepared us to pursue dynamic loading studies using hard x-ray techniques in the future FEL Sources. High-powered lasers that deliver femtosecond pulses can cause energy to be deposited into a material much faster than the characteristic relaxation times for the system to react. This leads to a confinement of large quantities of energy and consequent realization of dynamic compression of condensed matter. This also occurs during explosions, in the detonation of nuclear weapons, in inertial fusion experiments, or when a large meteorite hits Earth. The high pressures of a shock wave can generate thermomechanical extremes, for example make materials much denser than they were and heat them to thousands of degrees. Furthermore, dynamic shock conditions may induce novel structural configurations resulting in materials with entirely new emergent behavior. Understanding the dynamic pathways in the extreme transformational state of electrons, spins, phonons, nanoscopic to mesoscopic order, entanglement of lattice strains and defects, following the nucleation of the far-fromequilibrium state is one of the keys to successful materials control required in the next generation of technologies.

How does Li transform from its metallic state to become an insulator after dynamic shock loading? Can we measure the time evolution of pair-correlations in materials to further understand the new properties of condensed systems following a dynamic shock? Will we be able to measure the collective behavior of electrons, phonons, spins and atoms in condensed matter following extreme conditions of dynamic loading? Spatiotemporal studies following dynamic compression using next generation hard x-ray FELs will allow us to address these questions. 


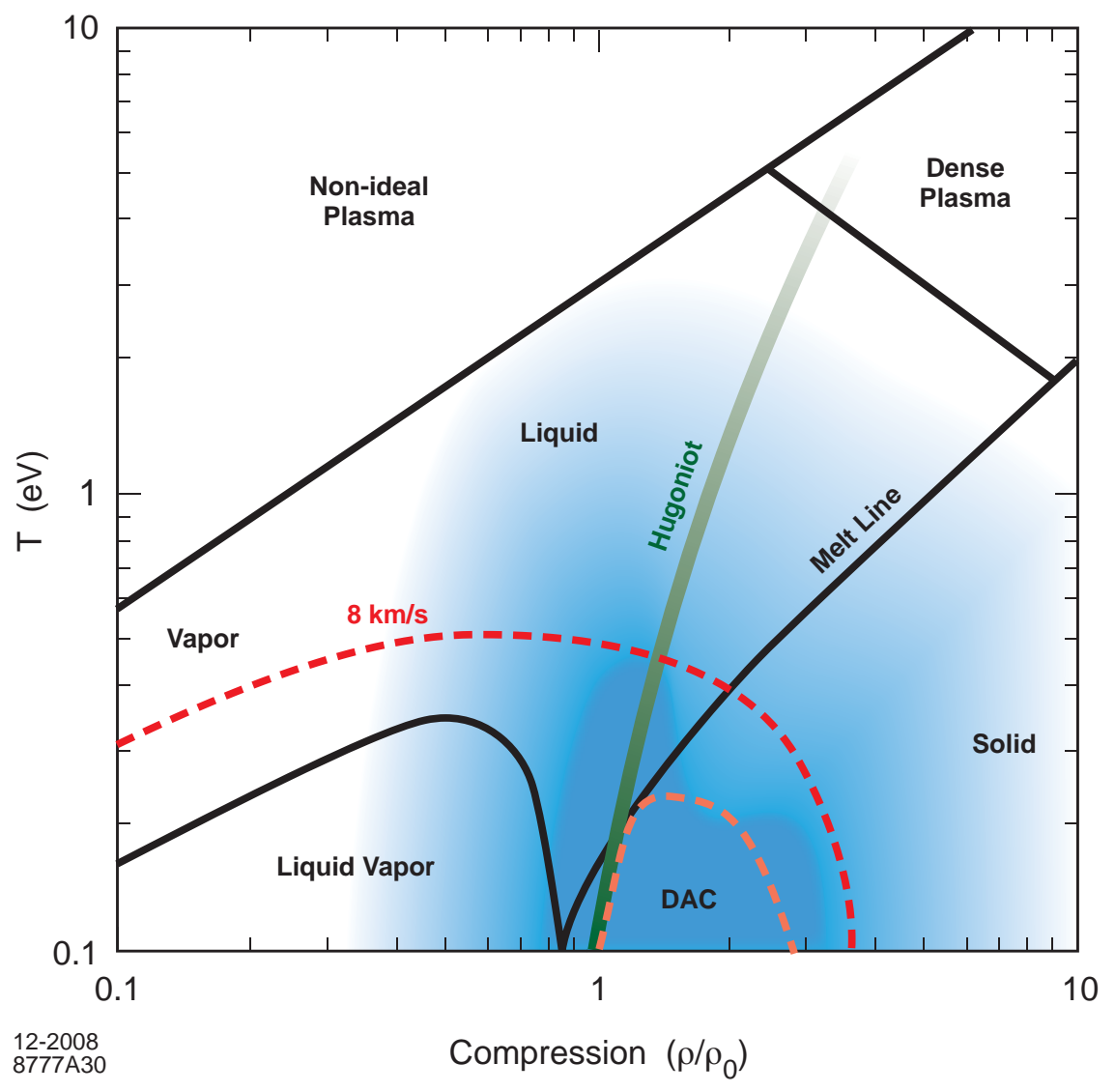

Figure 3.17. Dynamic compression allows us to probe new state of condensed matter with unusual properties. The static compression techniques using DAC will be limited in realizing the new forms of matter. The plot of temperature in $\mathrm{eV}$ and compression the potential new phases of matter. [Source: N. Holmes]

\section{$3 \quad$ Intense Electromagnetic Fields}

Electromagnetic excitations may be broadly classified by their field strengths or intensity (field strength squared) and time duration and/or frequency. For materials, a useful reference is provided by the bonding "field" experienced by valence electrons, which is of the order of 1 V/atom, corresponding to a field strength of $\sim 10^{10} \mathrm{~V} / \mathrm{m}$. Characteristic time scales in solids are set by nuclear motions ( $\left.>100 \mathrm{fs}\right)$ and valence electron motions ( $\sim \mathrm{fs}$ ). With our present and future capabilities to create electric fields that exceed the bonding fields and span a large frequency range from terahertz or picosecond fields to x-ray or attosecond fields we are entering new regimes of experiment and theory that go well beyond our present knowledge. This will require the development of new theoretical approaches that include dynamic field effects.

\section{Optical Excitation}

Femtosecond optical excitation of materials coupled with new probe sources capable of atomic-scale resolution opens up new possibilities for elucidating the fundamental structural and dynamical properties underlying nanoscale materials at extreme conditions, as illustrated in Figure 3.18. 

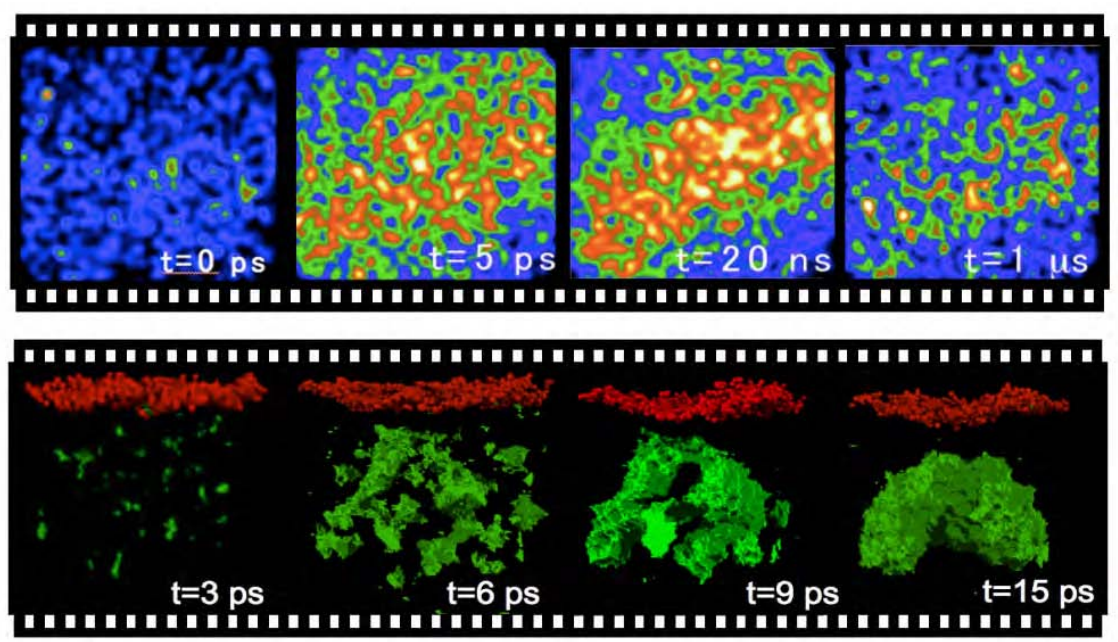

Figure 3.18. Top: Raw $x$-ray diffuse scattering measurements capturing the nucleation of nanoscale voids in optically excited semiconductors in real time. Bottom: molecular dynamics (MD) simulations showing the formation of nanoscale voids beneath the surface, supported by the experimental data. [Source: A. Lindenberg]

Atomic-scale rearrangements and synthesis of new structural forms may be enabled by the ability to characterize and capture snapshots of the transient states induced by high temperature and/or high pressure. It is largely unknown how phase diagrams at the nanoscale are modified. Techniques are required for traversing and accessing new regions of the phase diagram that are impossible to reach with present steady-state methods. This may be achieved through direct optical excitation of materials as well as through the generation of shock waves that rapidly traverse the material to be studied, both requiring transient techniques to characterize the structural evolution. Such studies are also critical for discovering new pathways for synthesis of materials of enhanced stability and strength.

Other key questions, with an eye on both fundamental and applied research, are: "How do complex phases involving spin-orbital-charge order respond to excitation with light? Why are strongly correlated electron systems so sensitive to photo-stimulation? How do these dynamic processes evolve in time? Can one put them to good use?”

\section{$X$-ray Excitation}

The interaction of materials with electromagnetic fields in the optical frequency range is accompanied by heating, melting or even plasma formation caused by photoexcited interband transitions. However, most of the interactions involve valence electrons. An exciting possibility is to use two x-ray sources in which one source has sufficient flux to create significant populations of excitations from core levels. Such photodoping experiments could be driven into new, potentially revolutionary directions. In particular, one may exploit a short x-ray pulse to populate empty orbitals, which could now be accessed from a core level to provide element and symmetry specificity, while using a second x-ray pulse to study the ensuing dynamics. One obvious example would address the magnetism of chemical bonds in complex oxides. The accepted paradigm of Goodenough-Kanamori rules connects the filling of particular orbitals with the sign and magnitude of the exchange interaction. Photo-doping could affect this on the ultrafast timescale, triggering collective rearrangements that dissect the elementary coupling mechanisms within the solid. More specifically, one question one could ask is, can one populate selectively the d-levels of a transition 
metal and impulsively flip the sign of the exchange interactions? How would such an exotic state of matter look like?

Other areas of interest may result from multi-photon spectroscopies, extending the NEXAFS technique to multi-photon excitations. In this way, one could access different selection rules for the NEXAFS lines, and understand the hybridization of solids in new ways. Pairs of FEL pulses could be used, for example, to study $\mathrm{p}$ and $\mathrm{d}$ character of a bond by studying linear and two-photon absorption. Other extensions of nonlinear optics could be used to study complex electronic properties, from hole-burning spectroscopy in the x-ray range to multi-dimensional studies of ultrafast electron dynamics.

\section{$\mathrm{THz}$ Excitation}

The far-infrared or terahertz spectral range consists of photons with meV energies that drive low frequency vibrations and intra-band electronic transitions, including collective phenomena such as charge density waves, excitons and superconductor pair-breaking. To date, nearly all experiments that study the ultrafast response of solids have been performed with visible or near visible excitation. Yet one often wishes to understand how a dynamically fluctuating ground state, perturbed on thermal energy scales, couples to the electronic structure. Most of the excitations that do affect the physics of these compounds occur at energies near or below room temperature, i.e. on energy scales well below $100 \mathrm{meV}$ corresponding to the $\mathrm{THz}$ spectral range. While THz laser systems have been available for decades, few have been capable of producing ultra-fast, single cycle pulses with intensities sufficient to induce measurable behaviors. As such, we are only beginning to explore the effects of high power $\mathrm{THz}$ fields on matter, as it has only recently become possible to produce ultra-strong half-cycle pulses of $\mathrm{THz}$ radiation with electric-field strengths rivaling bonding fields in materials ( V/atom) and magnetic fields exceeding typical laboratory fields (> 10 Tesla). Having access to such strong transient electric and magnetic fields, one can consider addressing some important questions such as "Can one switch, or modulate, the collective magnetic state of a complex oxide at $\mathrm{THz}$ frequencies? Can one photo-induce a quantum coherent state and, indeed, superconductivity? Can one control magnetic systems (and frustration) on the ultrafast timescale?

First experiments, conducted by exposing ferromagnetic thin films to strong transient magnetic fields, have revealed remarkable new physics that is presently poorly understood. The ultra-strong fields are found to lead to a nonlinear magnetic response that fractures the magnetization and imposes a speed limit on the technologically important magnetic-switching process. The electric fields are capable of distorting the atomic valence charge on the level of ligand field-like anisotropies and lead to a transient novel magneto-electronic anisotropy, possibly opening the door to manipulating the magnetization by electric fields alone. Most surprising is the absence of Joule heating in the sample. This indicates that extreme electric fields can temporarily transform a transition metal into a new electronic state, indicating physics beyond present models. These experiments are just the first experimental evidence for unusual behavior of matter in extreme THz fields. An entire field is open for exploration and, in particular, the combination of THz pump and x-ray probe experiments will offer the exciting opportunity to separately study the atomic, electronic and magnetic response of materials to extreme electromagnetic fields. A few specific opportunities are described below.

Recently, it was shown by the Cavalleri group that $15 \mathrm{THz}$ radiation could be used to directly excite a single IR-active vibration in various manganites and drive an ultrafast electronic, orbital transition. Such vibrationally driven transitions can be excited with a small fraction of the energy $(<1 \%)$ necessary to heat the sample to the transition temperature, directly modulating the distance of some selected bonds. Such 
experiments are then controlling the bandwidth of the solid on the ultrafast timescale. In a sense, these experiments are the ac equivalent of high-pressure studies. Yet, in these first phonon-induced phase transition experiments, only the mid-IR region near $17 \mathrm{THz}(17 \mu \mathrm{m})$ could be accessed, and only high frequency modes that stretched oxygen and metal bonds could be driven. In perovskite structures one would like to understand how stretching (15 THz), but also bending (10 THz) and external (5 THz) modes couple to the electronic structure, when excited coherently at large amplitudes, could be coupled to the electronic structure. In other systems, one is interested in selectively exciting superconducting gaps in the few $\mathrm{THz}$ range. Other collective excitations, like charge and spin fluctuations, or even Josephson plasma resonances could be driven at high amplitudes into the non-linear regime, and understand excitation, coupling to other modes. New approaches for the control of quantum coherence may result from these experiments.

Probing at x-ray wavelengths would enable measurements of the photo-induced pathways by tracking (a) the amplitude of the light induced distortions with hard-rays, (b) the rearrangements in orbital, spin and charge order with soft x-ray scattering and spectroscopy, (c) the rearrangement in the Fermi surface with time and angle-resolved photo-emission spectroscopy, (d) the evolution of domains by coherent imaging with femtosecond resolution.

One often encounters novel materials physics in the vicinity of phase boundaries. Materials such as ferroelectrics and multiferroics show spontaneous polarization (electric, magnetic and combination) below a critical temperature, sometimes associated with a structural distortion. A strong THz pulse could be used to drive the material through the relevant transition while the structure is probed by $\mathrm{x}$-rays to follow the dynamical evolution of any distortion (Figure 3.19). The picosecond time scale is particularly relevant since these distortions are usually evident as phonon mode softening into the THz spectral region. For applications that depend on switching of the ferroelectric or ferromagnetic state, the device speed is often limited by domain wall motion. Intense THz pump / x-ray probe studies may allow the fundamental limits for wall motion to be quantified.

Finally, we note that the electrical infrastructure for meeting the Nation's needs in transporting energy efficiently and reliably depends on high-strength dielectric materials and potentially (in the future) superconducting materials. The strong electric field of a THz pulse could be used to trigger a breakdown event and allow the initial process dynamics to be probed on an ultra-fast time scale. UV and soft x-ray spectroscopies would be useful probes of the valence and core level electronic structure and occupation as the breakdown process developed and evolved. Similarly, a strong transient E-field applied to a superconducting material will drive the flow of supercurrents and eventually disrupt the superconducting state. The dynamics of this process are unknown, but ultimately it should lead to a collapse of the gapstructure in the spectrum of electronic excitations and the loss of long-range phase order. If the process occurs on a time scale short compared to thermalization, disrupting superconductivity with a strong $\mathrm{THz}$ pulse may enable the study of material properties that are otherwise masked by the superconducting phase. 


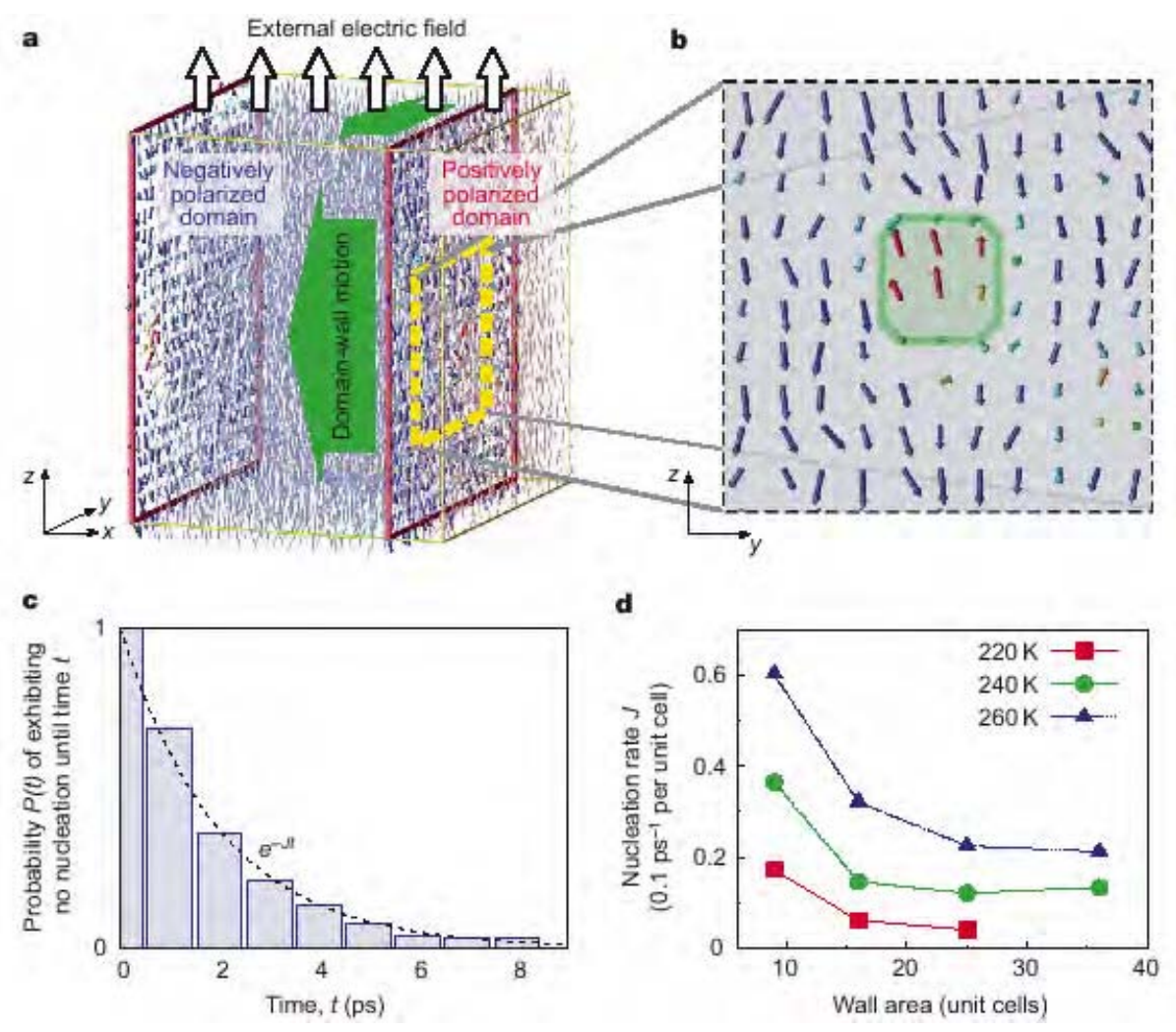

Figure 3.19. Calculation of ferroelectric domain dynamics for $\mathrm{PbTiO}_{3}$ under an applied electric field of $0.5 \mathrm{MV} / \mathrm{cm}$. Sub-picosecond rates are expected as the domain size approaches atomic dimensions [from Y-H Shin et al, Nature 449016165 (Oct 2007)].

\section{$4 \quad$ Polycrystalline Materials Science}

The realization of new high brilliance, short wavelength $\mathrm{x}$-ray sources would offer unprecedented capabilities to study real materials during service and deep within bulk materials, where behavior is fundamentally distinct from the behavior in near-surface regions. For example, spatially-resolved measurements with high-energy x-rays are a transformational class of synchrotron science with the potential to revolutionize engineering and applied sciences; spatially-resolved characterization of stress distributions, texture and particle size distributions that evolve near structural inhomogeneities can test and guide models that have the potential to dramatically improve materials performance.

An area currently unexplored is the detection and characterization of minor phases that emerge at interfaces of real materials such as complex phases at cladding interfaces in nuclear fuel rods, phases at the fiber/matrix interface in composite materials and in service measurements of corrosion including elastic stresses and studies of stress corrosion cracking. In addition to spatially-resolved measurements, timeresolved measurements will allow the discovery of transient phases that evolve during welding, heat treatment, concrete solidification, amorphization and other phase evolving processes.

Finally, high-brilliance, high-energy beams offer unique advantages for imaging. For example phasecontrast high-energy imaging and tomography can resolve structures that would otherwise be invisible to X-rays. Phase contrast imaging can also resolve internal structures in specimens comprised of mixed Z components or inside environmental chambers, and can characterize sprays and jet distributions that are 
difficult to study by alternative methods. With advanced time resolution, imaging will document the evolution of cracks, voids, precipitates and other phenomena in real time.

\section{$5 \quad$ What Is Needed?}

Today, $\mathrm{x}$ rays are among the most powerful tools to characterize materials in extreme conditions, but new sources will be necessary to push the methods into novel experimental regimes. Under conditions of intense pulsed excitation, extremely bright and ultrashort probe pulses spanning both soft and hard x rays are required. Studies of samples inside diamond anvil cells require the penetration of the probe into the pressure cell combined with its sensitivity to the local structure and dynamics. X-ray diffraction and inelastic hard $\mathrm{X}$-ray scattering from electronic and collective excitations are such probes, and sources having the highest possible average brightness at photon energies ranging from $7-30 \mathrm{keV}$ will be needed for the study of systems under extreme static pressures.

Ultra-fast laser systems for intense optical excitation exist, but will need to be matched and synchronized to the $x$-ray source probe pulses. In the case of x-ray pump, x-ray probe experiments, pairs of synchronized and independently tunable FEL's are necessary. These would require extreme temporal resolutions, few femtoseconds or even attoseconds, as one would often be clocking processes that involve the decay of short-lived core excitations.

$\mathrm{THz}$ pump, x-ray probe measurements will require $\mathrm{THz}$ electric fields reaching the $\mathrm{MV} / \mathrm{cm}$ level and beyond in order to create non-linear excitation and approach electric fields at the atomic scale. One also wishes to have $\mathrm{THz}$ fields with carrier-envelope phase stability to the probe pulses, in order to be able to interrogate the system at various phases of the electric field. Incorporating strong field $\mathrm{THz}$ pulses into $\mathrm{x}$ ray measurements will require the development of pump- probe instrumentation and techniques. We will also need the technology and experience for extracting, transporting, manipulating and characterizing intense $\mathrm{THz}$ pulses from accelerator and laser sources, and ultimately combining the THz with ultra-fast $\mathrm{x}$ ray probe sources to produce and sense the desired strong-field conditions at the sample.

High-brightness, hard x-ray $(20-80 \mathrm{keV})$ sources are needed for scattering and imaging. Hard x rays provide a unique probe for in situ, atomic-scale structural and chemical studies during materials synthesis. The nanoscale imaging capabilities possible with future coherent $x$-ray sources are needed for direct observation of materials synthesis processes on the atomic scale, and to develop the science underlying the discovery of new materials for energy applications. Spatially resolved hard x-ray CXDI with $1 \mathrm{~nm}$ resolution of zero-D and 1-D systems are required for understanding the self-assembly process, quantum structures and the functionality of nanomaterials. ERLs, oscillator or seeded high rep-rate XFEL sources can satisfy this requirement.

\section{E. Life Science and Soft Condensed Matter}

Life science and soft condensed matter research cover a broad range of scientific interests in a wide range of length scales, from atoms, molecules, and macromolecular assemblies to cells, tissues, and entire organisms. Ever since the discovery of x-rays by Rontgen, x-ray methods have been playing an increasingly more important role in life sciences as evidenced by medical x-ray imaging, small-angle and wide-angle x-ray scattering, and more recently, x-ray crystallography. The availability of synchrotron sources has greatly accelerated the advances in these areas, particularly in biological crystallography. Today synchrotron based x-ray crystallography accounts for over $85 \%$ of all biological macromolecular 
crystal structures deposited in the Protein Data Bank, revealing remarkably complex structures of individual proteins, nuclear acids, and their assemblies with atomic or close-to atomic details.

As life scientists work on larger and larger macromolecular complexes (molecular machines) and focus more attention on structure-function correlations, x-ray crystallography is hampered by the difficulty of obtaining suitable crystals large enough for conventional diffraction data collection. Thus, there exists a growing need to extend existing x-ray structural techniques to hard-to-crystallize bio-molecules and to non-periodic heterogeneous specimens at the functional cellular levels. For biological systems, imaging at the cellular level has become critically important in the post-genomic era as we work to determine the functionalities of a vast number of genes and gene products identified as a result of modern molecular biology techniques. Cellular imaging is complemented by hard x-ray imaging of larger biological structures, from multi-cell complexes such as tissues, to live organisms.

With advanced $x$-ray sources on the horizon, $x$-ray imaging and microscopy will play an important role in bridging the gap in structural imaging between molecular structures and biological organisms. It will complement other methods such as optical microscopy and cryo-electron microscopy to provide highresolution imaging for larger specimens. As the same time, high brightness x-rays available at these new sources will enable x-ray crystallography on submicron sized nano-crystallites or even few-unit-cell crystals, thus vastly increasing the range of biological molecules that can be studied by crystallographic methods.

\section{$1 \quad$ Crystallography on Very Small Crystals}

It has long been suggested that the use of very bright x-ray micro-beams (10-1 $\mu \mathrm{m}$ or smaller in diameter) would offer potential advantages for discovery science in the study of very small crystalline materials. In crystallization trials, very often microcrystallites are formed in the solution but do not grow into larger sizes. These microcrystals are either undetected in screenng or deemed unsuitable for conventional x-ray crystallography even at present synchrotron facilities. The high-brightness x-ray microbeams at future synchrotron facilities, including those under construction, will enable x-ray crystallography on such microcrystallites. This can be done by scanning a thin SiN window coated with flash-frozen microcrystals and collect crystallography data on each one of them when detected. Since eventually a microcrystallite may be damaged by radiation, it will be necessary to go through many microcrystals in order to obtain a complete dataset, much like what virus crystallographers used to do on unfrozen virus crystals. Such development will greatly extend applicability of x-ray crystallography to a much wider range of biological macromolecules that may be difficult to crystallize.

At the same time, techniques to obtain micron and submicron crystals are undergoing dramatic development. Microfluidic devices are undergoing rapid development to perform crystal screens with much reduced sample requirements. It is often possible to obtain conditions that produce small (submicron) crystalline "entities" too small to be studied with current sources but expected to be accessible to the bright micron or submicron x-ray beams of new sources. Another approach is the use of biological or chemical scaffolds that promote the formation of micro- to nano-sized crystals consisting of engineered lattices. A particularly interesting example is the use of a virus system that forms micron-sized protein crystals inside infected cells. These crystals form a highly ordered molecular scaffold for a protein that is to be studied. Crystal sizes range from a few microns to as small as about $200 \mathrm{~nm}$ and can be obtained as homogeneous populations and in large quantities. Thus, the need arises for development of x-ray sources 
that produce extremely bright, coherent beams with diameters in the submicron range with a brightness comparable to or exceeding today’s most powerful storage-ring-based light sources.

Clearly, conventional methods of crystal mounting cannot be used for submicron crystals. One approach is to scan the entire sample and then to piece together a complete diffraction data set from crystal "hits" within the sample. Ultra-fast data collection is now possible with pixel array detectors (PADs). Crystal "mounting" may also take novel forms, such as using laser tweezers, simply passing the object through the beam using a capillary injector, or mounting samples on micro-fabricated grids that are then translated in the beam. Data collection is most efficient if the object's position in the beam can be timed to coincide with the x-ray pulse. For reproducible, mass-selected samples this arrangement may take the form of "serial crystallography," proposed by Spence and colleagues. "Orienting” may be by use of lasers or other external fields, or by detection of the directionality of molecular fragments. Phasing could be accomplished by iterative algorithms developed for diffraction microscopy (see below).

In summary, development and successful deployment of the new paradigms mentioned above for "submicron" crystallography will have a wide-ranging and broad impact. Achieving an understanding of the structure of large macromolecules and their complexes including membrane-bound proteins, how the interactions between macromolecules in these larger assemblies confer and control function (i.e., the workings of "molecular machines"), and ultimately how these assemblies are organized and networked at the cell and tissue level will lead to a knowledge base that will provide for a predictive understanding of complex biological systems from the sub-nanometer scale up through the cellular level. Such information would transform medicine, renewable energy research, environmental remediation, and other fields that are informed by or harness biological systems.

\section{In-situ Studies of Conformational Changes}

Conformational changes in macromolecules and their complexes are of enormous interest, as scientists try to understand protein folding, the functioning of enzymes, molecular machines, transcription, etc. Smalland wide-angle $x$-ray scattering have provided much information in this area but have been limited in time resolution to the millisecond and slower range in most cases. By using nanofluidic mixers, fast area detectors, as well as pump-probe techniques, high-brightness x-ray nanobeams will extend the time resolution well into the critical microsecond range and perhaps below. At this time scale, molecular dynamics calculations begin to overlap the observations, leading to a much more accurate description of the processes involved. This technique promises to revolutionize the study of the dynamics in soft matter in general, in ways that should have both fundamental (phase transitions and self-assemblies) and technologically important (mixing) applications. Given a highly parallel micron-size beam of hard x-rays, the steps in macromolecular folding can be followed on a microsecond time scale at nanometer resolution using a nanofluidic mixer and temperature-jump- or photo-activation, as illustrated in Figure 3.20. 


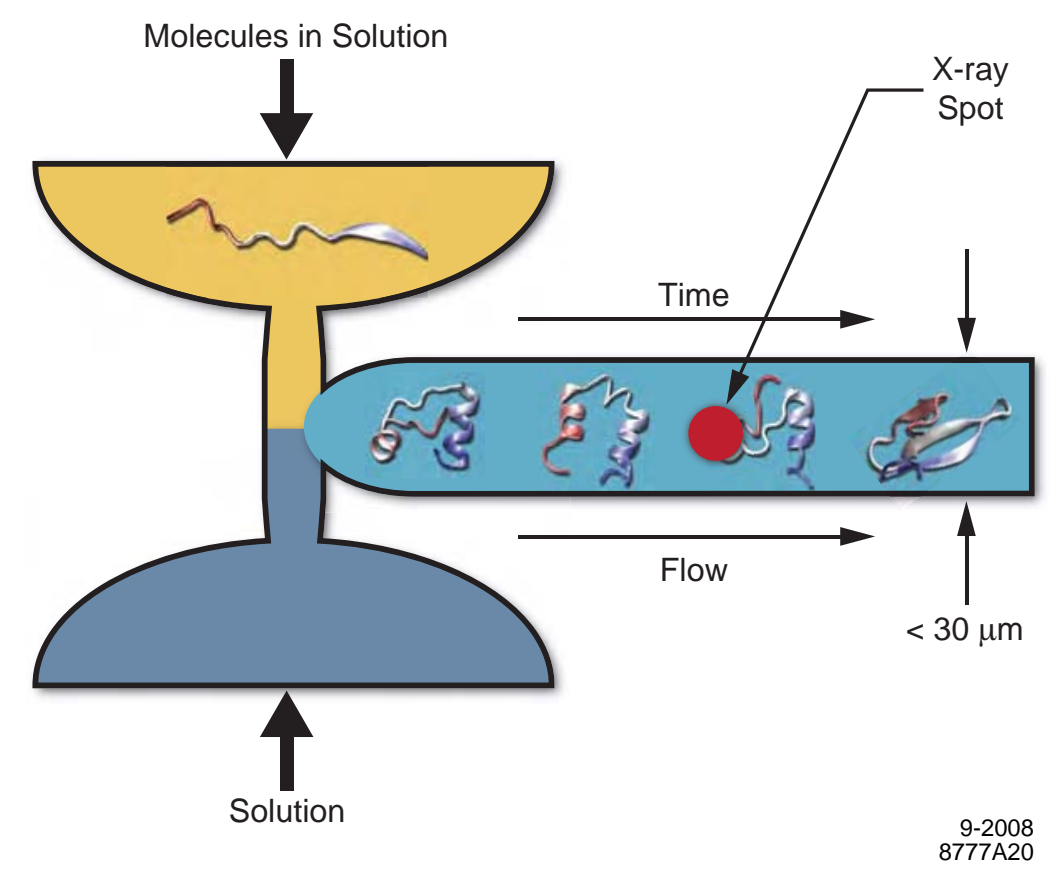

Figure 3.20. Schematics of a time-resolved x-ray scattering experiment using a nano-fluidic continuous-flow mixer [Matsumoto et al., JACS 129, 3840 (2007)]. An unfolded-protein solution (top) is mixed with a second solution (bottom) that promotes folding. The $x$-ray beam interacts with the solution jet at different distances from the mixing point, corresponding to different time points in the protein folding process [The folded protein models adopted from an MD simulation by Freddolino et al.,. Biophys. J. 94, L75 (2008)].

\section{$3 \quad$ Nanoscale Structure Dynamics}

There is growing evidence that conformational fluctuations in proteins and macromolecular complexes play a fundamental role in target recognition and functional switching. Dynamical structural techniques to supplement "static" atomic resolution structures are thus crucial in all aspects of structural molecular biology and in gaining an understanding of the relationship between structure and function. X-ray photon correlation spectroscopy (XPCS) is one such technique that has been highly effective in studying slow non-equilibrium phenomena in soft condensed matter. It requires coherent beams, and is therefore limited in terms of the parameter space it can reach today and in the mid-term future. Because signal-to-noise for this technique scales quadratically with incident beam intensity, it will clearly benefit from the more intense coherent beams available at future high brightness x-ray sources, including those under construction, which will enable dynamical studies on the microsecond to nanosecond time scales from optimized high brightness storage rings and at even faster time scales from FELs. These studies would substantially advance our understanding of biologically relevant dynamics as well as soft-condensedmatter dynamics at their natural time scales.

One example of such new areas of research would be proteins in solutions. Access to faster dynamics and the ability to probe more weakly scattering samples with XPCS at optimized high brightness synchrotron sources will open the possibility to study fluctuations involving large-scale conformational changes that can occur down to microsecond time scales. Such fluctuations are potentially important for protein function such as enzymatic activity. Another biological system for which XPCS could make significant contributions is biological membranes. For example, reflectivity studies that access microsecond-scale times would be ideally suited for characterizing the dispersion relations and damping of long-wavelength 
undulation modes in lipid bilayer systems, which provide direct access to the viscoelastic parameters of the membranes (e.g., the bending and compression moduli). By probing microsecond-scale correlations at the appropriate wave vectors, XPCS measurements at future facilities could potentially access the elastic properties at length scales of 10's of nanometers matching typical protein-protein separations that are relevant to biological function.

\section{$4 \quad$ Structural Biology at the Cellular Level}

How are macromolecular complexes organized into organelles and organelles organized within the cell? Much of what we know in this area comes from electron microscopy. While it provides superb resolution, it is limited to very thin samples. In contrast, visible-light microscopes are limited in resolution. X-ray microscopy, especially cryo-X-ray tomography and coherent diffraction microscopy, has been developing rapidly with the goal to fill the gap between electron and visible-light microscopies. Cryo-X-ray tomography is a very powerful new technique providing exciting results at the 30-50 nm resolution level in intact cells, limited by available x-ray optics. To go beyond this limitation, coherent diffraction microscopy being developed at several laboratories makes use of a fully coherent x-ray beam to generate a diffraction pattern from the specimen and uses a computer algorithm to invert the diffraction pattern to a real space image. Recent developments in ptychography, a technique that combines coherent diffraction with a scanning x-ray microprobe, promises to extend coherent diffraction microscopy to studies of larger, extended specimens, such as biological tissue sections and other higher order, hierarchical, biological structures. These lensless forms of 3D x-ray microscopy using intense, coherent $\mathrm{x}$-ray beams require the development of dedicated coherent $\mathrm{x}$-ray facilities at high brightness $\mathrm{x}$-ray sources.

Coherent beams from FELs will revolutionize these approaches. Illumination with an ultrashort and extremely bright x-ray pulse can outrun key damage processes over a very short period of time (Figure 3.21). This can be exploited to extend the diffraction signal to the highest possible resolution in flash diffraction experiments where we expect to achieve resolutions well beyond those limited by conventional damage barriers. A detailed analysis of imaging live cells with x-ray lasers suggests that nanometer to subnanometer resolutions could be achieved with intense, ultrashort x-ray pulses at wavelengths of about 1.5$0.5 \mathrm{~nm}$. This is substantially higher than resolutions possible in any other type of biological imaging experiments on non-reproducible objects. A high-resolution 3D data set from a non-reproducible object would require simultaneous flash illumination by techniques to be developed, e.g., with many pulses from many directions. This could, in principle, assure that significant damage does not develop during data collection. Orthogonal illumination could be realized with split-beam geometry with soft x-ray pulses for which efficient multilayer mirrors exist, and possibly with hard x-ray pulses using Bragg reflection beam splitters. 

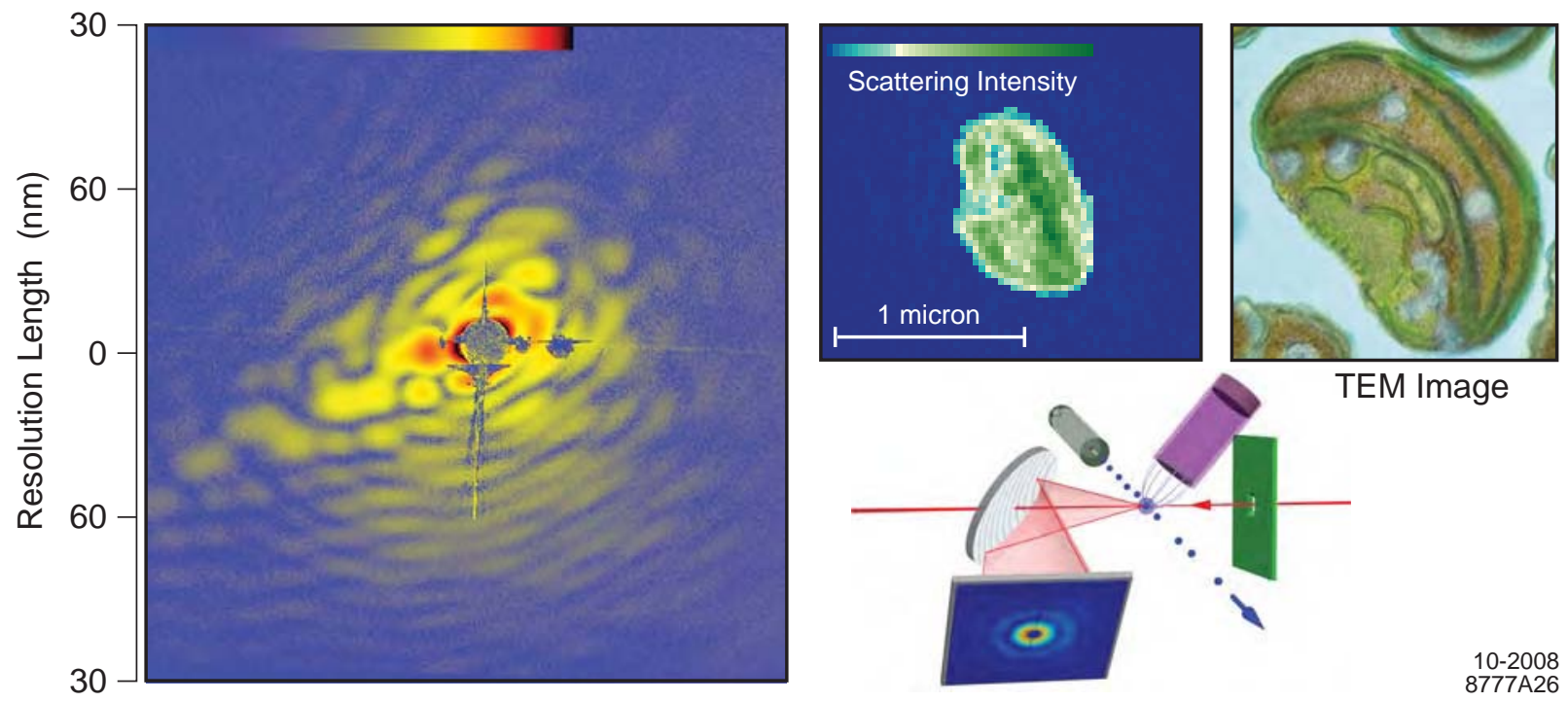

Figure 3.21. Left: Femtosecond single-shot x-ray scattering pattern. Top middle: The reconstructed image of a live picoplankton that was injected at $200 \mathrm{~m} / \mathrm{s}$ into the beam of the soft x-ray laser FLASH operating at 7-nm wavelength, 15-fs pulse duration, and $\sim 10^{14} \mathrm{~W} / \mathrm{cm}^{2}$ power density. Image reconstructed using Shrinkwrap (Sebastien Boutet). [Source: J. Hajdu et al., private communication] Top right: transmission electron microscopy (TEM) image. [Source: W. Eikrem and J. Throndsen, Univ. of Oslo]. Bottom right: Schematic setup of diffraction experiment. The soft x-ray image can be extended to molecular resolutions with an $\mathrm{x}$-ray laser operating in the wavelength range from $1.5-0.5 \mathrm{~nm}$.

\section{$5 \quad$ Trace Element Mapping}

X-ray fluorescence microscopy coupled with x-ray absorption spectroscopy techniques offers superb traceelement sensitivity and the ability to determine chemical speciation and local electronic and geometric structure of metal constituents in biological systems. To understand the biological significance of trace elements (especially metals) in health, disease, and pharmacological treatment, it is important to understand their distribution, concentration, and chemical state in cells and tissues as well as their many roles as key components of proteins and macromolecular complexes. For example, typically $25-30 \%$ of a given genome codes for proteins that contain or involve metals. Important first steps toward the highresolution mapping of trace elements have been taken at third-generation light sources, as shown in Figure 3.22, but restrictions in source brightness and x-ray optics resolution have limited the processes to date.

Higher average-brightness x-ray sources, nanometer-scale x-ray focusing optics, and dedicated traceelement mapping facilities with cryo capabilities are required to bring this technique into the forefront of cell biology, metal toxicology, pharmacology, and physiology. Higher brightness will also enable the study of metal-containing systems at in-vivo concentrations and under conditions where they are actually undergoing reactions and changes in the sub-millisecond time domain. In addition, nanometer-scale spatial resolution combined with the use of genetically encoded metal-binding tags would open up studies of subcellular localization of critical proteins in biological cells and organelles, thus providing the much needed link to understand protein functions. 


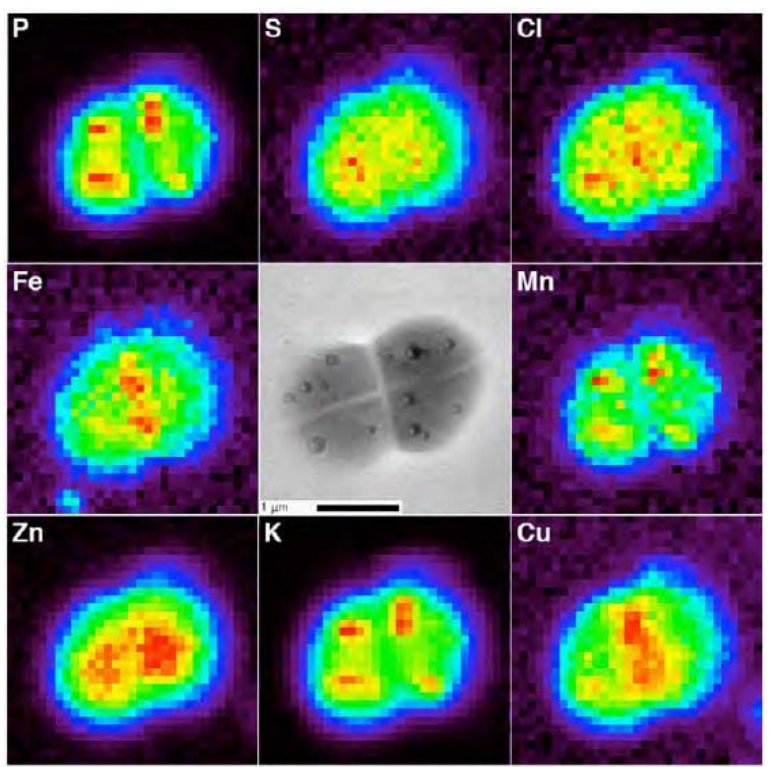

Figure 3.22. Elemental maps of radiation-resistant bacteria undergoing cell division, measured by $\mathrm{x}$-ray fluorescence microscopy at APS with a spatial resolution of $200 \mathrm{~nm}$ [Daly et al., PLoS Biol. 5, 92 (2007)]. The opportunity to achieve nm-scale resolution at optimized high-average-brightness sources in the future will greatly enhance our ability to understand the precise location of the metal elements with respect to subcelluar regions in these and other biological cells.

\section{$6 \quad$ Soft Condensed Matter and Biomaterials}

Soft condensed matter science and nano-biomaterials science reach into the fundamental as well as the applied science arenas. This broad field encompasses many novel and important application opportunities. Examples include block copolymer films, which can self-assemble into periodic domains on the order of 10 nanometers, offering an interesting route to nano-lithography. Solution-processable conjugated polymers are being developed for thin film transistors and photovoltaic devices. Biological membranes exist at aqueous-aqueous interfaces and provide a structural and dynamical platform for cell processes, but our understanding is only in its infancy. High-speed liquid jets see application in agriculture, energy, medicine, materials processing and combustion. Porous polymer-based semiconductor resins are considered for low dielectric constant applications for faster interconnects in integrated circuits. In all of these, high-energy $\mathrm{x}$ rays are an invaluable characterization probe, offering minimal beam damage and maximal access to multiscale analysis. Future x-ray sources are needed to offer the brilliance, stability and coherence levels for critical evaluation of multi-scale microstructures, surface atomic and molecular-level structures, dynamics and elemental sensitivity to bring soft matter and biomaterials science to the next level. Figure 3.23 shows the relevant length scales for synthetic, biological and hybrid soft matter, and current instrumentation for imaging at these length scales. 


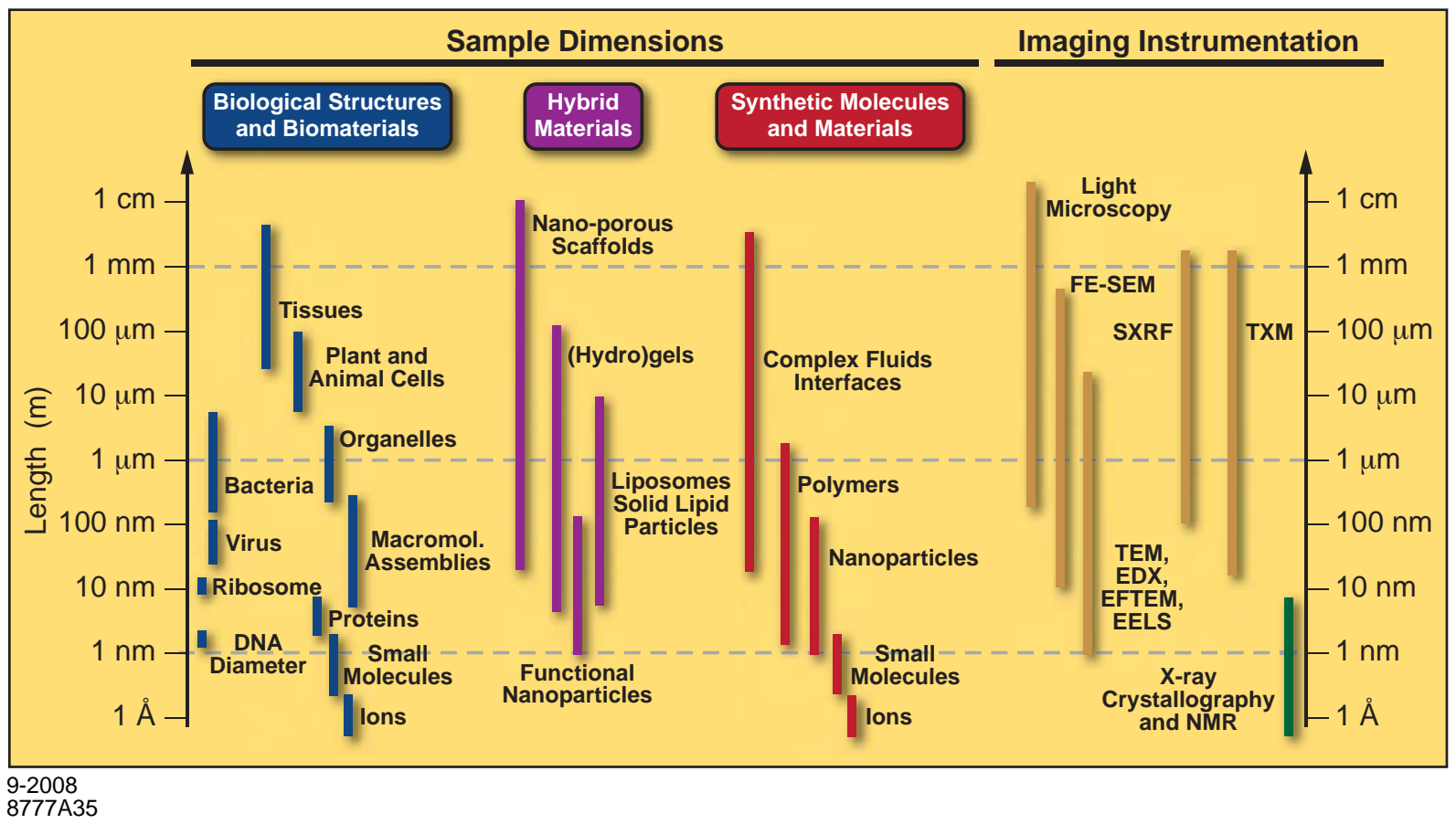

Figure 3.23. Scheme of relevant length scales in soft matter, and the available instrumentation for imaging their structures. [Source: K. Shull et al. APS Science Case Report on Polymers and Soft Materials (2008)]

\section{$7 \quad$ Damage Considerations}

In all areas of life science and soft matter research with x-rays, radiation damage is a critical limitation. As one moves to higher resolution, smaller samples, and higher sensitivity, one needs to be even more careful in the design of the experiment to make sure that the information collected is not compromised. There is considerable effort going into understanding these issues and finding mitigation strategies. In some cases, making the exposure at cryogenic temperatures is sufficient. In other cases a useful approach is to continually refresh samples, as with nanofluidics, a stream of molecules, droplets, or nanocrystals. Or, with sources of sufficient peak brightness and short duration, the information may be collected before the damage manifests itself. In all cases tested so far, there is evidence from imaging experiments at soft x-ray FELs that this technique can work.

\section{$8 \quad$ What Is Needed?}

To attain the goals in atomic-resolution crystallography and SAXS, a stable, ultrabright, hard x-ray source based on highly optimized synchrotrons and beyond is needed to provide highly parallel, micron, or submicron-sized beams. For fluorescence mapping, a similar source and cryo capabilities are needed to create and to make use of an intense hard x-ray nanoprobe with nanometer-scale spatial resolution. Coherent x-ray diffraction imaging and x-ray photon correlation spectroscopy on biological specimens require an intense coherent $x$-ray beam from stable high rep-rate hard $x$-ray sources such as a highly optimized synchrotron or an ERL. Flash imaging and ultrafast photon correlation spectroscopy require an ultrashort-pulse FEL with repetition rate adjustable from a few $\mathrm{Hz}$ to $100 \mathrm{KHz}$. 
THREE

Examples of Scientific Drivers for Future X-Ray Sources

52 WHITE PAPER 


\section{X-Ray Source Types, Capabilities, and Their Trade-Offs}

\section{A. Types of X-Ray Sources with Enhanced Capabilities}

In this section, we briefly discuss the major options for new sources in the 10-20 year time frame, based on the essential new x-ray capabilities that were described in Section 2.B, and what we believe is realistic. Figures 4.1 through 4.4 below summarize the approximate performance of present and future sources, including storage rings, energy-recovery linacs (ERLs) and linac-based FELs, based on projections from the present state of the art. While undulators are the primary source of photons for all sources described here, their performance characteristics are strongly linked to the accelerator technology used, and we group our discussion in terms of accelerator type accordingly. A combination of ring-based sources, including storage rings and ERLs, and linac-based FELs is identified as meeting the needs described in Section 2.B. A broader discussion of source options, including novel approaches that require considerable R\&D before becoming practical, is included in Appendix A. Technical areas requiring R\&D are addressed in Appendix B.

\section{Storage Rings}

Storage rings provide highly stable average flux and brightness with low peak flux and brightness at very high pulse-repetition rates (typically hundreds of $\mathrm{MHz}$ ) in a tunable photon energy range from the far IR to hard x-ray. Megahertz photon pulse rates, or even sub-megahertz rates for large rings, are possible using fewer bunches, and lower rates for timing or pump-probe experiments can be reached using a beam chopper. Pulse lengths are typically greater than ten picoseconds (rms); shorter pulse lengths can be reached at low current with lattice tuning and at high current with RF deflection systems and laser slicing. Advanced third-generation storage ring sources such as NSLS-II are optimized to achieve high average spectral brightness via a low electron-beam emittance of $0.5 \mathrm{~nm} \cdot$ rad. "Ultimate" designs offer exceptional brightness by pushing down the electron-beam emittance even further to $\sim 0.1 \mathrm{~nm} \cdot$ rad or less with very large circumference lattices and by supporting relatively high current, although high current operation presents challenges for high power absorber and optics designs. Even present facilities reach the diffraction-limited emittance for 1 - $\AA$ radiation ( $8 \mathrm{pm} \cdot \mathrm{rad}$ ) in the vertical plane by reducing horizontalvertical coupling and residual vertical dispersion. While ultimate rings would have higher peak brightness than most third-generation rings, some existing and in-construction rings reach ultimate ring peak brightness levels using long undulators and by storing a large current in a small number of bunches. With sufficiently bright beams partial x-ray lasing may be achieved, resulting in an amplification of emission by one or two orders of magnitude through the self-amplified spontaneous emission (SASE) process. Storage rings support a large user community with several tens of beam lines at each facility. Storage-ring technology and implementation methods are presently the most mature and reliable for multi-user facilities. 


\section{Energy-Recovery Linacs (ERLs)}

ERLs, now the target of R\&D for future light source use, promise to provide very high average brightness by circulating a very low-emittance $(\sim 10 \mathrm{pm} \cdot \mathrm{rad})$, low-energy-spread (order $\left.10^{-4}\right)$ round beam from a fullenergy superconducting linac for one turn around a ring-like transport line containing insertion devices. The beam is then returned to the linac, where the beam energy is recovered in the superconducting structure. Because the beam is not stored in the ring, the very low beam emittance from the linac, which actually decreases with increasing beam energy, is approximately preserved in the single turn around the ring, and performance limitations associated with ultimate storage rings, such as injection accumulation, lifetime, and intrabeam scattering, are avoided. Very high brightness with low current is reached by virtue of the very low emittance, reducing the total synchrotron radiation power load but also the photon flux, as compared with storage rings of similar brightness and higher current. While the bunch length is on the order of $1 \mathrm{ps}$ in the high-current ( 100 mA), high-brightness/coherence mode, a short-bunch mode is envisioned that would provide bunch lengths of tens of femtoseconds at the expense of much lower current and higher emittance. Bunch repetition rate may be adjusted from the $\mathrm{MHz}$ or less level for short-bunch, high-charge operation up to the linac frequency (potentially $\mathrm{GHz}$ ) for high-current, high-brightness operation. The low energy spread enables an effective gain in brightness from high-order undulator harmonics and from long insertion devices $(\sim 25 \mathrm{~m})$, but the energy spread must remain small to sustain the energy recovery in the linac. Emittance and energy spread increase as the beam travels from the first to last insertion device, especially in a short-bunch mode, reducing source brightness from the downstream devices accordingly. Low-energy ERLs are being used to drive infrared FELs, while high-energy ERLs would provide sufficiently small emittances and peak currents to drive SASE and possibly cavity oscillator FELs (XFELOs—see Section 4.A.3).

\section{Linac-Based FELs}

Linac-based FELs will provide short x-ray pulses of unprecedented peak brightness and power, up to tens of GW per pulse, and photon energies from the far IR to hard x-ray. The first x-ray FELs will utilize SASE, producing pulses with very high spatial coherence and some degree of temporal coherence to provide line widths of $\sim 10^{-4}$. An FEL can also be used as an amplifier, as an oscillator, and as a harmonic generator. Employing seeding and other optical manipulations with conventional lasers such as high-gain harmonic generation (HGHG), harmonic cascade, self-seeding, electron-bunch slicing and/or length and charge optimization, and x-ray FEL oscillator (XFELO, or regenerative amplifier FEL-RAFEL or harmonic cascade FEL ) configurations, exquisite control of the x-ray pulse properties can be achieved in principle. A single linac can drive multiple FELs, each with independent tunability, and each configured for specific capabilities. X-ray pulses of 10-100 femtoseconds, and even sub-femtosecond pulses could be generated, as well as long (picosecond) pulses with near transform-limit temporal coherence (meV bandwidth).

The electron source for FELs can be a pulsed room temperature or superconducting linac, or a CW superconducting linac. Photon energy is dependent primarily on machine energy and FEL configuration, and not necessarily determined by type of linac used. The FELs under construction today achieve the highenergy electron beam required to radiate in the hard x-ray range using pulsed linacs with repetition rates for the macro pulses of $\sim 10-120 \mathrm{~Hz}$. Pulsed linacs operating in bunch train mode can provide high bunch repetition rates over the duration of the linac pulse length. Future CW superconducting linac-based FELs promise to provide high pulse repetition rate with even spacing, from tens of $\mathrm{kHz}$ to $\mathrm{MHz}$ or greater as electron gun technology is developed, and offer a route to higher average brightness with a time-structured 
beam that is well suited to many experimental needs, particularly pump-probe. The development of CW SCRF technology, also used in ERLs, offers improved stability over pulsed machines, allowing the use of broadband feedback loops to control beam position and energy.

\section{Graphical Representation of Source Capabilities}

Brightness: X-ray source brightness is defined as photon density in 6-dimensional phase space, with phase space dimensions defined by horizontal and vertical beam size and divergence, time and fractional bandwidth of energy (or frequency). The usual units for brightness are photons $/ \mathrm{s} / \mathrm{mm}^{2} / \mathrm{mrad}^{2} / 0.1 \%$ bandwidth. Average and peak brightness of various source types are compared in Figure 4.1.

Figure 4.1. Composite envelopes indicative of the approximate range of average (a) and peak (b) spectral brightness for present and potential future technology source types. The envelopes are not inclusive of all sources that exist or are envisioned. The high average brightness from the linac- and ERL-based FELs, all of which have low repetition rate compared to ring- and ERL-based spontaneous sources (see Figure 4.2), is not usable by a large class of experiments due to the associated very high peak brightness. Brightness of the seeded FEL sources is increased by their intrinsically narrow bandwidth, which can approach the transform limit, and can be several orders smaller than incoherent sources (see Figure 4.4). The CW linac soft $x$-ray FEL envelopes represent spectra for beam from a fixed linac configuration; hard $x$-ray FEL envelopes represent estimates for a variety of source configurations. ERLbased, single-pass FELs may achieve peak brightness comparable to the $\mathrm{CW}$ and pulsed linac FELs, and average brightness comparable to the $\mathrm{CW}$ linac FELs indicated. See text below for discussion of assumptions made in this figure.

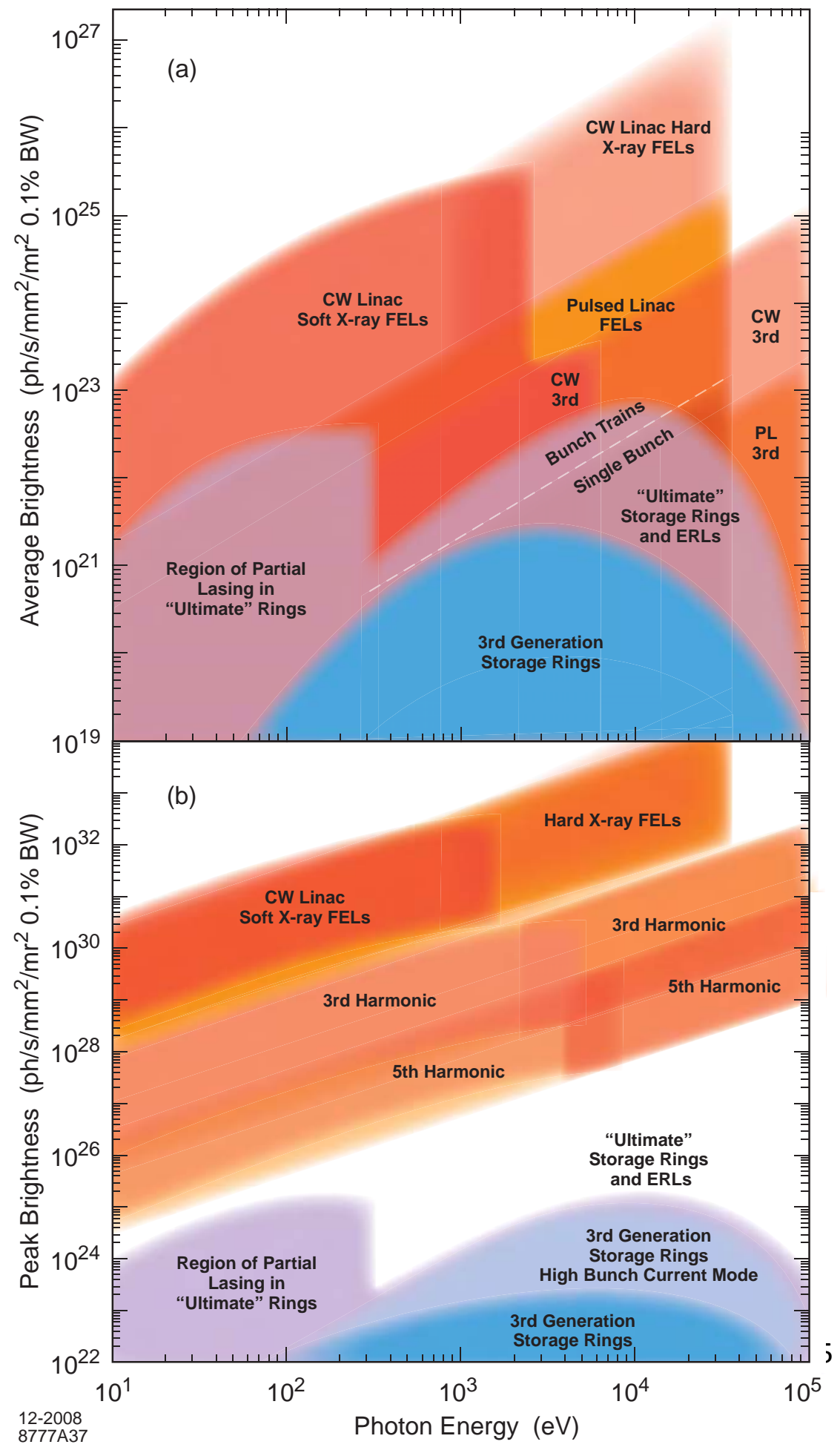


In Figure 4.1, future-technology ERL performance assumes a 25-m undulator in high-brightness mode; 3.5-8-m undulators are assumed for large-circumference "ultimate" ring performance. Peak brightness from advanced third-generation ring sources having long undulators $(\sim 20 \mathrm{~m})$ and operating with a large current per bunch (> 3 $\mathrm{mA} /$ bunch) in a small number of bunches approaches that shown for "ultimate" rings having shorter undulators and much less current per bunch. The peak brightness from long undulators on "ultimate" rings would be greater than shown if they too were operated with greater current per bunch. Pulsed FELs are presently in construction. CW linac FELs and ERLs require future technology; notional designs indicated are for a 100-kHz bunch rate CW linac soft x-ray FEL and a 1.3-GHz bunch rate hard x-ray ERL. The range shown for FEL performance reflects SASE and seeded modes of operation and ability to tune performance over a wide range. The peak brightness for FELs is several orders of magnitude greater than incoherent sources, resulting in high average brightness even at low repetition rate. The average brightness from pulsed linac FELs can be increased by operating with a large number of closely-spaced bunches within the RF pulse of the linac, resulting in a "burst-mode" time structure-in this notional design 4000 bunches spaced by 200 ns repeating at $10 \mathrm{~Hz}$. For FELs and sub-10 pm·rad emittance ERLs, the flux is spatially coherent; some FELs may have a high degree of temporal coherence. The potential brightness range of a seeded hard x-ray FEL is represented by an X-ray FEL cavity oscillator (XFELO) in Figure 4.1, in this case based on a 1-MHz pulse repetition rate with low-charge $(<\sim 50 \mathrm{pC})$ picosecond electron bunches provided by a CW linac, multipass recirculating linac, or an ERL, and an Xray optical cavity tuning system using four crystals movable in position and angle. Other types of seeded hard x-ray FELs using pulsed linacs or high energy CW linacs could have comparable performance to the XFELO. The depicted upper energy limits for linac-based FELs are not absolute; they may be extended with higher-energy linacs. The lower energy bound for all sources extends to the sub-eV (THz) range.

While linac-based FEL sources can achieve high average brightness with bunch repetition rates much lower than those of rings and ERLs as a result of their coherent radiation, this fact has to be interpreted with care. The potential drawbacks of operating linac FEL sources in high average brightness mode, including possible sample damage, are discussed in Section 4.B and in Appendix C.

Pulse Structure: The temporal characteristics of different sources are illustrated in Figure 4.2. The indicated notional designs indicated are for a $10-\mathrm{kHz}$ bunch rate $\mathrm{CW}$ linac soft x-ray FEL and a 1.3-GHz bunch rate hard x-ray ERL. The number of photons per pulse for FELs is dependent on wavelength and electron-beam energy. The range in FEL performance reflects SASE and seeded modes of operation, and ability to tune performance over a wide range. For pulsed FEL bunch trains, the average repetition rate is calculated assuming 4000 bunches spaced by $200 \mathrm{~ns}$, repeating at $10 \mathrm{~Hz}$. The region shown for a seeded XFEL depicts performance estimates for an XFELO based on a 1-MHz pulse repetition rate with lowcharge $(<\sim 50 \mathrm{pC})$ picosecond electron bunches provided by a 7-10 GeV CW linac. 


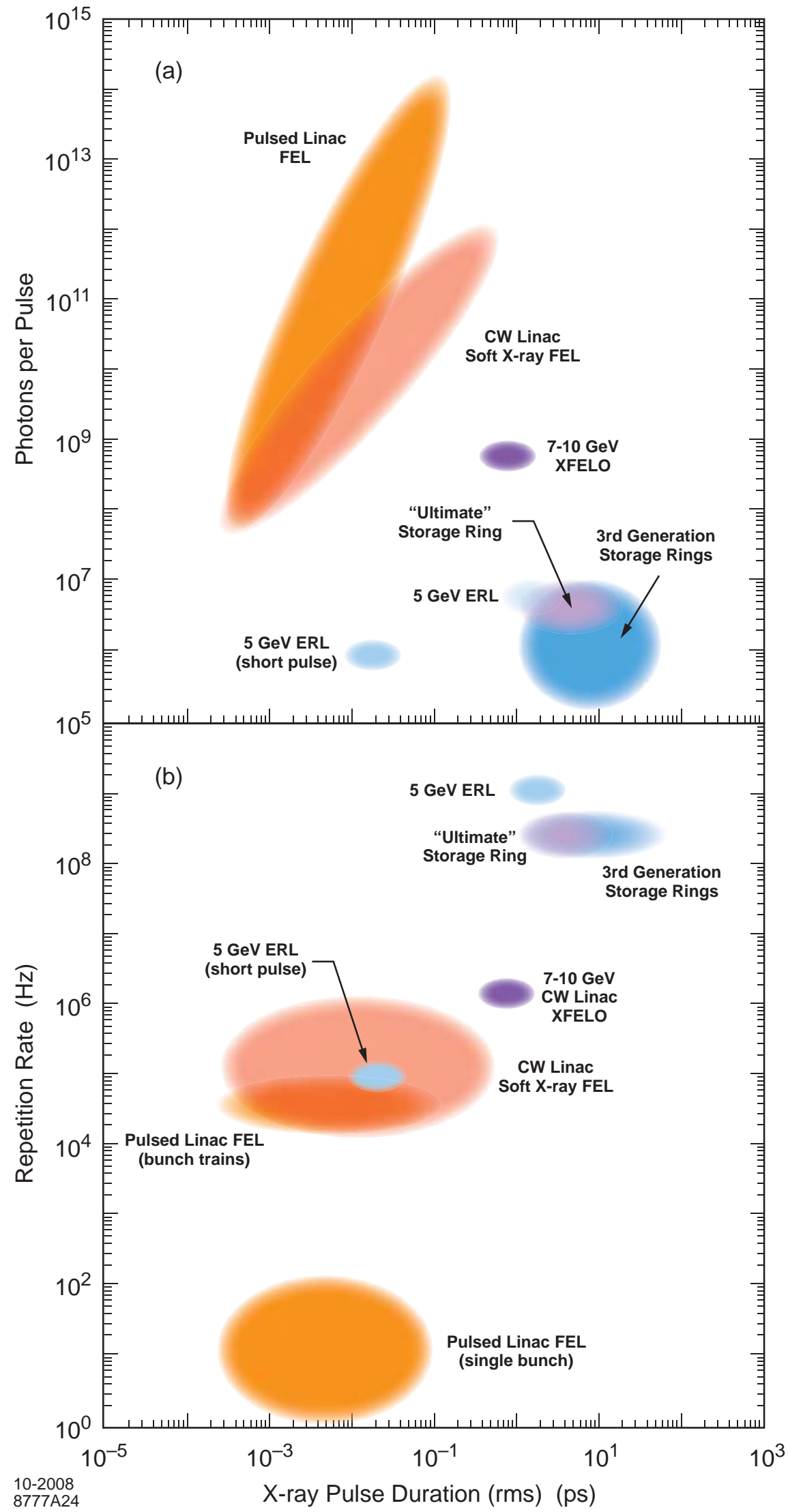

Figure 4.2. Envelopes indicative of the approximate performance ranges of present and future technology source types illustrating the trade-offs between peak and average performance parameters as discussed in the text. The envelopes are not inclusive of all sources that exist or are envisioned. Future FEL performance may encompass a wide range including the envelopes shown, dependent on details of design. See text for discussion of assumptions made in this figure. 
Transform Limits: The quality of a photon source may be characterized by two limits, characterizing a perfectly coherent source. The "diffraction-limited" emittance $\varepsilon_{\mathrm{ph}}$ of a photon beam expresses the fundamental coupling of transverse source size and angular divergence in the space-momentum domain and defines complete spatial coherence. For a Gaussian beam, $\varepsilon_{\mathrm{ph}}=\lambda / 4 \pi$. For spontaneous radiation generated from magnets in an electron accelerator, the photon beam emittance for a given wavelength is a function of source magnet properties (i.e. undulator or dipole magnet parameters) and of the electron beam emittance. In effect, the radiated photon emittance at a given wavelength is a convolution of the diffraction limited photon emittance and the electron emittance. As the electron emittance in one transverse plane approaches the diffraction limit for a given wavelength, the degree of spatial coherence, or coherence fraction, of the radiated photons in that plane increases, reaching the order of $50 \%$ from undulators when the electron emittance reaches the diffraction limit, subject to the matching of the electron beam emittance ellipse to the diffraction-limited photon beam ellipse. The coherent fraction increases towards $100 \%$ as the electron emittance is reduced below the diffraction limit. In contrast, the amplified radiation from FEL sources is $100 \%$ transversely coherent and diffraction-limited in both planes when the emittance of the round electron driving beam is close to or, for sufficiently long undulators, even greater than he diffraction limit, by virtue of "gain guiding", where only a single diffraction-limited radiation mode is amplified to saturation in the FEL process.

In Figure 4.3 we have plotted the electron beam emittance versus photon energy for the different undulator-based sources of Figures 4.1 and 4.2.

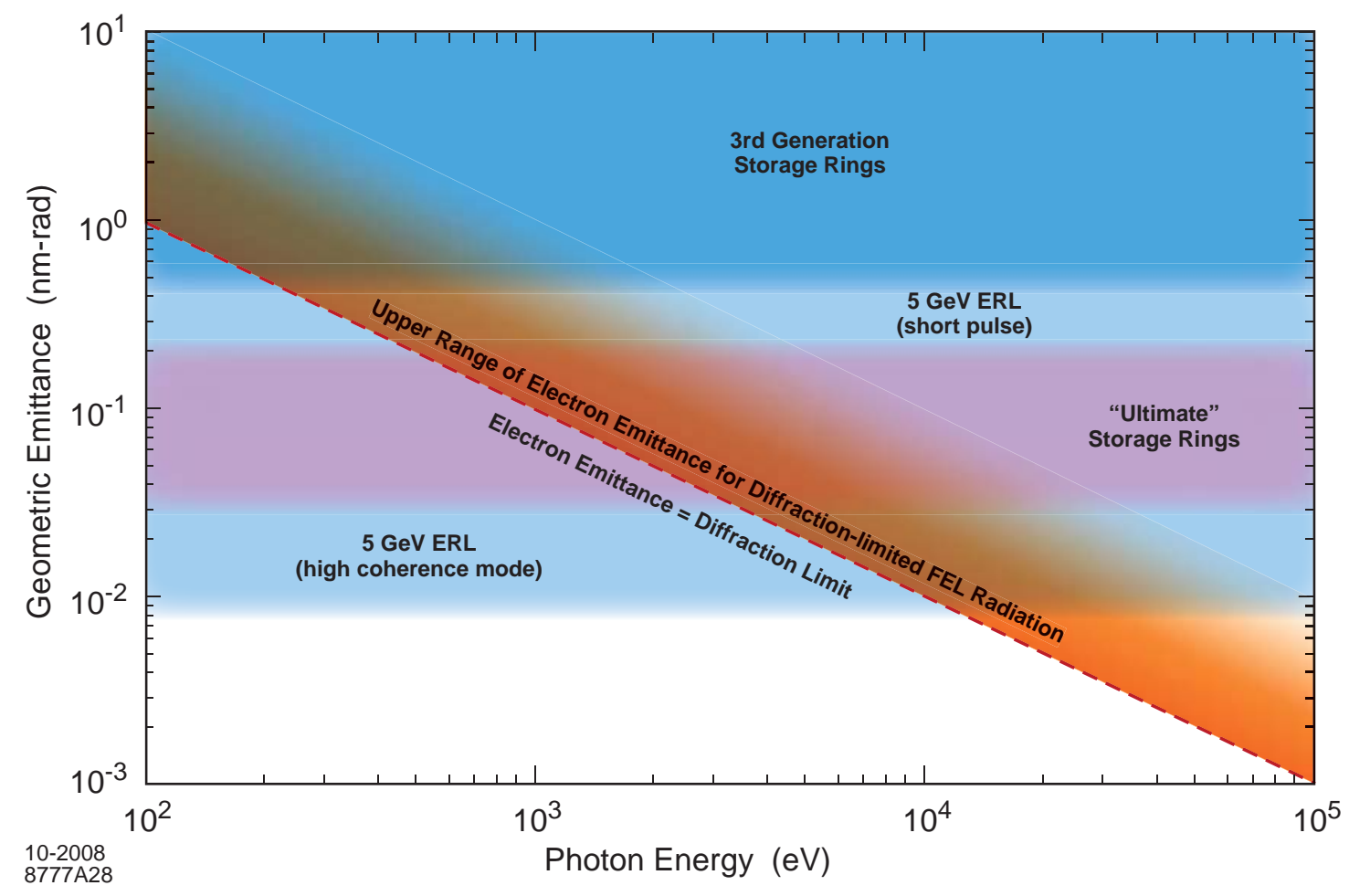

Figure 4.3. Envelopes indicative of the approximate emittance ranges of present and future technology source types. The transversely coherent fraction at a given photon energy for spontaneous radiation sources on storage rings and ERLs approaches $100 \%$ as electron emittance is reduced below the diffraction limit. Radiation from single-pass FELs having sufficiently long undulators is $100 \%$ transversely coherent even if the driving electron beam emittance is somewhat larger than the diffraction limit. 
Just as the "diffraction limit" characterizes complete spatial coherence, the "transform limit" (also called the "Fourier transform limit" or "bandwidth limit") defines complete temporal coherence. It expresses the fundamental coupling between energy resolution and time resolution, and is described by the product of time duration and energy width of the $\mathrm{x}$-ray pulse, and for a Gaussian pulse intensity is given by $\Delta \mathrm{t} \cdot \Delta \mathrm{E}=$ $\mathrm{h} / 4 \pi=0.33 \times 10^{-3}$ [ps (rms) eV (rms)].

In Figure 4.4, the bandwidth attainable with coherent and incoherent undulator-based sources is indicated for the notional designs discussed in Figures 4.1 and 4.2 and accompanying text.

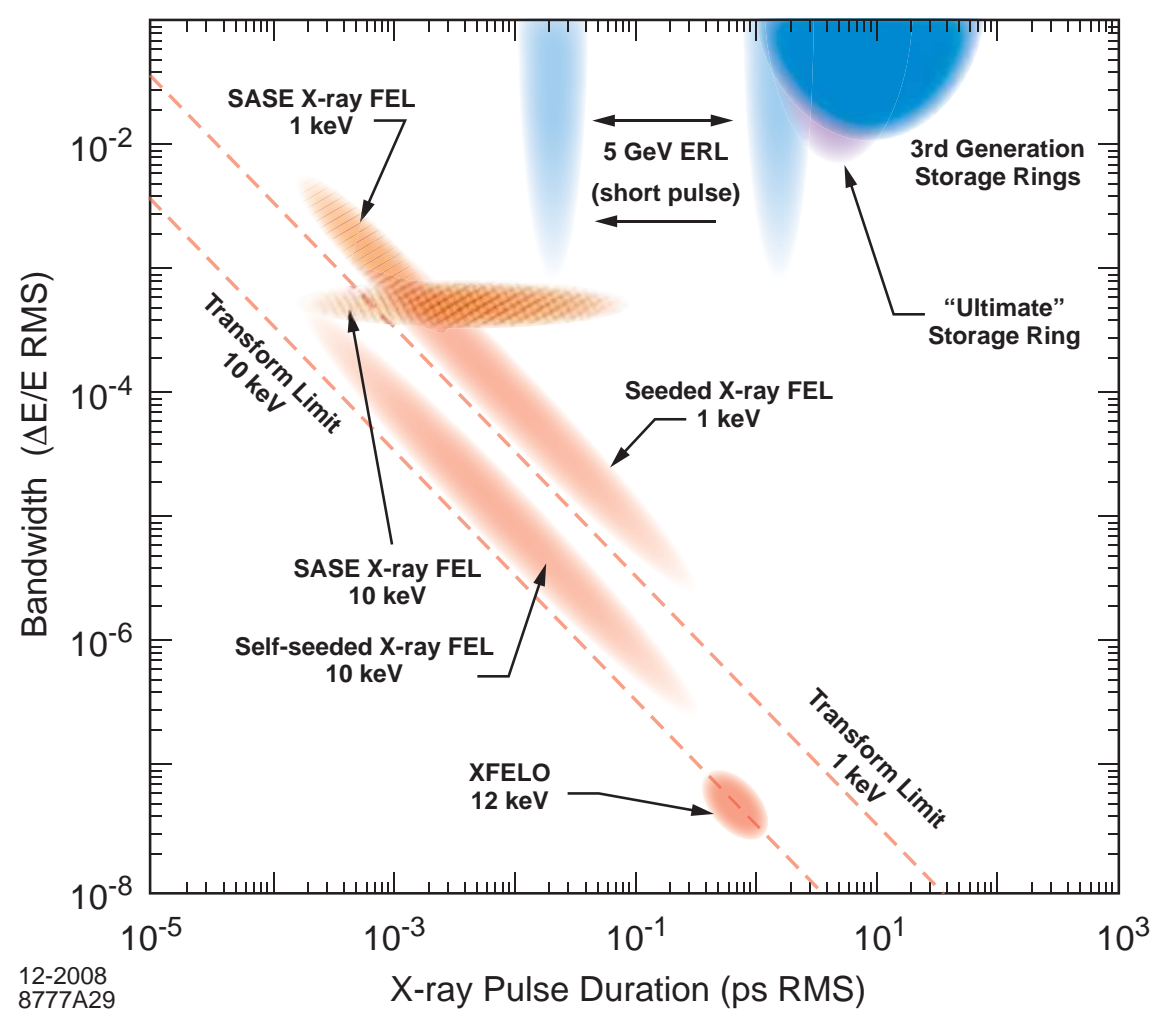

Figure 4.4. Envelopes illustrating fractional bandwidth capabilities of undulator-based sources in present and future x-ray facilities. Bandwidth is given for direct output from the source with no additional wavelength filtering. Dashed lines indicate the transform limit for $1 \mathrm{keV}$ and $10 \mathrm{keV}$ photon energies. Seeded FELs and oscillator FELs can have bandwidths approaching the transform limit, as can SASE FELs with very short bunches. Photon energies are indicated for specific examples used to illustrate coherent sources. Narrow bandwidth contributes to increased brightness. See text below for discussion of assumptions made in this figure.

In practice, a transform-limited pulse has the shortest possible duration for a given energy bandwidth or resolution. Transform-limited pulses yield the highest brightness for a given total pulse energy, and are important for extremely brightness-hungry experiments such as those involving coherent multi-photon (nonlinear) processes. They are also required for phase-controlled pulse shaping techniques to be used.

Seeded FELs and FEL oscillators with long bunches can approach the transform limit with very narrow bandwidths. For these sources, deviations in the electron phase-space distribution along a bunch are expected to limit the bandwidth to a factor of a few above the transform limit. The seeded FEL envelopes in Figure 4.4 represent external laser seed and self-seeding capabilities, for FELs with 1-keV and 10-keV photon energies. The XFELO example in Figure 4.4 corresponds to a 12-keV photon energy FEL oscillator with narrow bandwidth provided by relatively long electron bunches; other types of seeded hard 
X-ray FELs could have comparable performance to the oscillator if using similar bunch lengths. SASE FEL envelopes are shown for photon energies of $1 \mathrm{keV}$ and $10 \mathrm{keV}$. For SASE, the bandwidth is determined by the cooperation length (the distance over which the radiation field moves forward from the electrons in one gain length), not the bunch length. For ultra-short bunches the cooperation length can approach the bunch length, and the (large) bandwidth can be close to the transform limit. For ring and ERL-based sources, undulators radiate the smallest intrinsic fractional bandwidth (given by $\Delta \mathrm{E} / \mathrm{E} \sim 1 / \mathrm{N}_{\mathrm{u}}$ where $\mathrm{N}_{\mathrm{u}}$ is the number of undulator periods), over a very wide range of photon energies (from a few eV to tens of $\mathrm{keV}$ ), with bandwidth down to a lower limit imposed by the coherent energy spread in the electron beam (typically $>10^{-3}$ for rings and $>10^{-4}$ for ERLs). This characteristic of ring-based sources, and the much broader spectrum offered by dipole magnet radiation (where $\mathrm{N}_{\mathrm{u}} \sim 1$ ), can have advantages in experiments where a large bandwidth is required. For large bandwidth sources, the bandwidth of radiation for an experiment is typically determined by that of the beam line's energy selection and filtering device, such as a crystal monochromator, and is therefore an adjustable parameter, which can be advantageous for some experiments. In contrast, this bandwidth selection is not possible for many FEL sources, although their very narrow bandwidths can eliminate the need for monochromators.

\section{B. Discussion of Relative Capabilities and Trade-Offs}

In the discussion of x-ray source performance capabilities begun in Section 4.A and continued in Appendix A, brightness (both peak and average), coherence, pulse structure and transform limits have been used as metrics. While the relative advantages and disadvantages of source types might be assessed using these metrics, the choice of a preferred source for any given experiment is ultimately based on maximizing the number of usable photons delivered in the phase-space acceptance of the experiment within the data acquisition period of its detector system, and is thus experiment specific. Some experiments demand the high peak brightness and coherence of an FEL source; others demand high flux in a relatively large acceptance phase space and are therefore not entirely brightness limited. Such experiments would benefit from a higher current ring-based source. As another example, experiments demanding very high energy resolution might not benefit from ultra-short pulses, while those demanding high time resolution would benefit. The breadth of beam property requirements for present and future $\mathrm{x}$ ray science ensures that, given our present understanding, a combination of linac-based FEL and ringbased sources will be required to accomplish the x-ray science of the future. The relative capabilities and trade-offs of these new sources are discussed below.

The spectral brightness characteristics of the sources discussed in Section 4.A are compared in Figure 4.1, which reveals that FEL sources are not only superior in peak brightness but also have larger average brightness than storage ring sources. The FEL advantage in peak brightness arises because the FEL amplification process produces a very large number of photons in each pulse (see Figure 4.2), and packs these photons into a small (diffraction-limited) phase space. The high average brightness arises because the FEL peak brightness is so great that it more than compensates for the low repetition rate. FELs have another advantage over ring-based sources, due to the fact that their electron pulses are prepared by a linac and then used only once to create x-rays. This allows, in principle, precise control over each individual xray pulse. For example, pulses with a useful number of photons within durations of less than about $10 \mathrm{fs}$ can only be achieved with FEL sources (see Figure 4.2). The large number of photons per pulse can be an advantage for any single-pulse experiment, where the experimental conditions are not repeatable (see Section 3.D above). At a more profound level, the greater coherence of FEL x-rays compared to spontaneous x-rays could enable new research techniques involving nonlinear x-ray interactions. FELs 
therefore offer a range of x-ray capabilities that is unique and cannot be covered by storage ring or ERL sources.

One may be led to conclude that all future sources should be FELs. The situation is however considerably more complex, as already briefly mentioned in Section 3.C.5, and further discussed in Appendix C. Many experiments will be unable to exploit the high average brightness of an FEL, because they will be overwhelmed by the huge peak brightness. Limitations may arise from practical matters such as instantaneous detector count rates, or from sample "damage" (significant modification of the sample properties by the FEL pulse during the measurement, see Appendix C). FEL bunch trains may exacerbate these difficulties, if the spacing of the bunch train does not allow synchronization with pump lasers, sample relaxation or recovery, or replenishment or translation. Also, not all experiments are improved by added brightness; for many the figure of merit is simply the flux into the (fixed) sample volume as discussed above.

Ring-based sources (storage rings and ERLs) provide high average flux and brightness with low peak flux and brightness. They support tunable devices that provide photon pulses at very high repetition rates, and may be considered "CW" sources for many applications. In addition, storage-ring-based sources now incorporate more highly developed technologies for stabilizing source position and angle than do linacdriven sources, relying on the static nature and periodicity of storage rings. (Nonetheless, for a linac the position and angle can be maintained to a fraction of the radiation pulse radius and angular divergence.) Storage ring-based sources are proven technology, have well-established user communities, and are essential to a broad range of science; the next-generation storage rings use mature technology, and are ready to be built.

Only the combined capabilities of ring-based and FEL sources provide the complete range of x-ray characteristics required to address the grand challenges and to meet the broad set of science requirements with the wide range of technologies users need. A combination of FELs of different kinds (SASE, seeded, and oscillator configurations have unique and complementary characteristics) and storage-ring or ERL sources will provide the "total information" needed to understand complex and emerging phenomena in quantum matter and detailed dynamics of chemical reactions and biological processes.

Existing storage-ring spontaneous $\mathrm{x}$-ray sources can produce a maximum photon density that is equal to the value of the fine-structure constant, or about 0.01 photons per "coherence volume", a unit of phase space whose 6 dimensions satisfy transform limits (sometimes referred to as the degeneracy parameter). Note that this is simply another way of normalizing the spectral brightness, or of describing how far the pulse is from the transform limit in which all photons are in the same mode. Because ring-based sources produce low-degeneracy x-rays, they are not well suited for exploiting nonlinear x-ray effects. ERLs produce photons from a smaller phase-space volume, and may have degeneracy of 100; FEL sources, through the coherent amplification process, produce highly degenerate $\mathrm{x}$-ray pulses $\left(10^{7}\right.$ or more photons per coherence volume, with further orders of magnitude increased degeneracy obtainable from seeded and oscillator FELs). The high degeneracy available from FELs is relevant for any non-linear effect requiring the simultaneous presence of multiple coherent photons within a short pulse. In principle, this should enable the extension into the x-ray range of nonlinear techniques (frequency doubling, 4-wave mixing, etc.) that are used routinely in the optical frequency range. For these techniques to be generally useful, it would also be necessary to identify non-linear x-ray processes with reasonable cross-section. This research, and the exploitation of nonlinear x-ray effects, falls within the range of science described in Section 3.A. 
FOUR

X-Ray Source Types, Capabilities, and Their Trade-Offs

62 WHITE PAPER 


\section{Other Considerations}

\section{A. U.S. X-Ray Sources in the International Environment}

In the last five years, there has been a great effort worldwide to construct and develop x-ray light sources culminating in the successful completion of a number of new third-generation sources, in the upgrade and improvement of several others, and in the construction and development of the first fourth-generation sources. The relevance of such activity is clearly evident in Figure 5.1 that shows the proposed and funded $\mathrm{x}$-ray light sources and R\&D facilities around the world.

Several of the new third-generation light sources have been successfully commissioned (DIAMOND in the UK, SOLEIL in France, ASP in Australia, and SPEAR III at SLAC), and a number of them are under construction (SSRF in China, TPS in Taiwan, ALBA in Spain,, and NSLS II at BNL) . Groups at some of the older light sources are now proposing significant facility upgrades and modifications that will enhance their source performance (APS at ANL, ALS at LBNL, ESRF in France, and Spring-8 in Japan), and at former collider facilities conversion to high-brightness light sources is under way (PETRA III at DESY in Germany) or in conceptual planning (PEP-X at SLAC). Significant effort worldwide is aimed at bringing in the next decade the ring-based sources from the present brightness of $\sim 10^{19}$ B.U. (B.U. = brightness unit $=$ photons $/ \mathrm{s} / \mathrm{mm}^{2} / \mathrm{mrad}^{2} / 0.1 \%$ BW) above $10^{22}$ B.U., towards their ultimate limit near $10^{23}$ B.U.

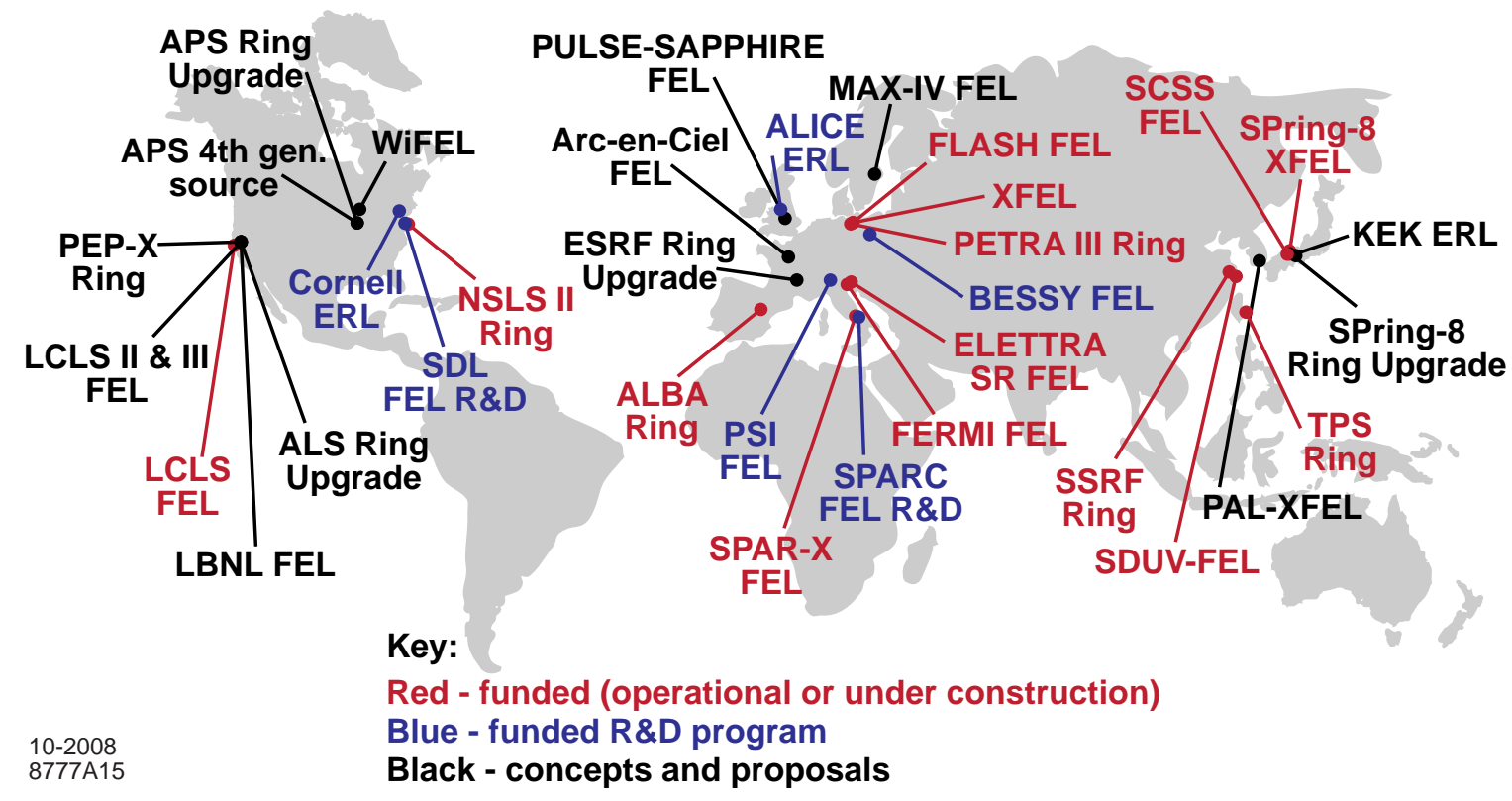

Figure 5.1. Proposed and funded $x$-ray light sources and R\&D facilities around the world. 
In parallel, interest in a "fourth generation" of light sources has shown a sharp increase in the international communities of users and accelerator physicists. FELs offer revolutionary performance, including peak brightness of more than $10^{33}$ B.U. and sub-picosecond pulse lengths, open the door to new types of experiments, and nicely complement the outstanding science results in the consolidated fields investigated by the large and steadily growing user communities of third-generation light sources. ERLs with their very high average brightness exceeding $10^{22}$ B.U. and short bunch capabilities, together with "ultimate" storage rings, represent a natural extension of third-generation light sources beyond their present performance limits. In fact, some of the ERL concepts under development include an existing storage ring in their layout.

The first VUV-soft x-ray fourth-generation light source user facility, FLASH in Hamburg Germany, operating between 50 and $6.5 \mathrm{~nm}$, has demonstrated the ability of an FEL-based source to perform as a reliable facility and is already generating significant science results. Recently the SCSS Test Accelerator in Harima, Japan, has opened a user program operating in the 50 to 60 -nm range and will be developed further to reach the 3 to 4 -nm range. In the hard x-ray portion of the spectrum, LCLS at SLAC (1.5 $\AA$ ) is presently under commissioning, the European XFEL (1.0 $\AA$ ) has been launched with construction to be in Hamburg Germany, and the SPring-8 Joint Project for XFEL is building a 1.0- $\AA$ facility in Harima, Japan.

The broad international activity in light source construction, development, and planning highlights today's strong user support, as well as competition, for the development of next-generation light sources. In the construction and upgrade of third-generation light sources, Europe and Asia have invested and are continuing to invest large resources. A few years ago, the U.S. upgraded SPEAR III at SLAC and recently funded the construction of NSLS-II at BNL, thereby taking very important steps towards maintaining the U.S. competitiveness in the field. Pursuing the same objective in the future requires the continuing upgrade of the veteran U.S. facilities as well as the construction of new facilities.

Activities in the development of VUV and soft x-ray FEL facilities are dramatically growing as well. In addition to the already operating FLASH, a large number of laboratories around the world are proposing or developing concepts for FEL facilities in this wavelength range (LBNL FEL at LBNL, BESSY-FEL in Germany, WiFEL in Wisconsin, SAPHIRE and PULSE in the U.K., Arc-En-Ciel in France, MAX IV FEL in Sweden, and PAL in South Korea). The SPARX project in Italy and the SDUV in China has been funded, and FERMI@Elettra is presently under construction. An interesting characteristic of soft x-ray FELs is that their lower photon energy makes the use of seeding techniques appealing and more realistic, and in fact the majority of the projects include seeding schemes for improving the temporal and spectral characteristics of the photon pulses.

Preserving the U.S. competitiveness in the case of soft x-ray FEL sources requires effective short-term action in supporting R\&D which will allow implementation of advanced $\mathrm{x}$-ray facility capabilities within a decade. In Europe and Japan, several FEL R\&D facilities have been funded and some are already in construction. In the U.S. the activity is in an initial proposal phase where innovative ideas and creative schemes using state-of-the-art techniques and beyond are ready to be exploited if the projects are funded. Support for initiatives within the U.S. can allow us to compete with and perhaps lead the world in such an important field.

Concerning hard x-ray FELs, the U.S. is presently at the forefront of the field with the LCLS scheduled to begin operations in summer 2009. In order to maintain this leadership when the XFEL in Europe and the SPring-8 XFEL in Japan start operating, development and upgrades of the LCLS as well as new initiatives 
in the field are worth considering. ANL is considering fourth-generation sources, including an $\mathrm{x}$-ray FEL oscillator and an ERL. R\&D on a high-brightness electron injector and $250 \mathrm{MeV}$ accelerator at PSI in Switzerland is leading toward design of a hard x-ray facility reaching $1.0 \AA$.

Development of x-ray ERL facility concepts is also underway. After the successful operation of smallerscale and longer-wavelength ERL-based facilities (JLAB FEL in Virginia, JAERI in Japan, and the ERL at BINP in Russia), Arc-En-Ciel has a 2-GeV ERL option, and the studies at Cornell and at KEK and JAEA in Japan for the construction of 5-GeV hard x-ray facilities with average brightness exceeding $10^{22}$ B.U. and below 100-fs bunch length capabilities are in an advanced stage of study with R\&D efforts in progress. The Cornell layout includes the existing CESR ring, and the APS group at ANL have discussed a similar ERL machine as a possible long-term upgrade for their facility. R\&D on ERL technologies (also relevant to FELs) is in progress at the ALICE facility in the U.K. The U.S. effort is led by the funded R\&D program at Cornell. The main international competitor is the ERL being studied at KEK and JAEA in Japan and Arc-En-Ciel in France. Currently the US has a lead in design and prototyping for an ERL, but faces stiff competition from the foreign efforts.

In competition with developmental ERL-based x-ray sources, and sharing the user interest and support for developing high average brightness, low peak brightness, high repetition rate sources, are "ultimate" storage rings. While these rings have longer bunches with larger energy spread than ERLs, they have brightnesses similar to ERLs, achieved with more conventional technology by virtue of their large circumferences, enabling very low emittance lattice implementations, and, in some cases, also due to their higher operating current (Section 4.A.1 and Appendix A.1). Ultimate rings are being investigated by SPring-8, the APS and most recently by SLAC, which would use the 2.2-km tunnel, injector and much of the accelerator technology developed for the recently closed PEP-II B-Factory.

The broad interest in next-generation light sources is also evident from the number of R\&D facilities proposed and operating around the world to investigate critical subsystems and techniques used in FEL or ERL schemes. These include among others, high-brightness electron guns, emittance compensation and manipulation, seeding techniques, laser synchronization and ultra-stable timing distribution systems, laser and cathode development, and electron and photo beam diagnostics. The Source Development Laboratory (SDL) at the NSLS is a $300 \mathrm{MeV}$ linac-based high brightness electron beam and free electron laser (FEL) laboratory capable of testing novel FEL concepts such as cascaded high gain harmonic generation (HGHG), ESASE (Enhanced Self Amplified Spontaneous Emission), and critical intense electron beam dynamics issues such as longitudinal space charge, coherent synchrotron radiation, and laser heaters. Other R\&D facilities such as SPARC in Italy and the SCSS test accelerator in Japan are also in operation; the MAX-Lab seeding facility in Sweden and ALICE in U.K. are being commissioned; the PSI FEL R\&D in Switzerland has been already funded; and others such as STARS in Germany and APEX at LBNL, have been proposed. Cornell is commissioning a prototype ERL gun and injector, KEK is preparing for a test ERL loop, and JAEA is developing an ERL high current and ulta-low emittance gun. Appendix B highlights $\mathrm{x}$-ray source $\mathrm{R} \& \mathrm{D}$ requirements.

\section{B. Growth in the U.S. User Community}

One of the great strengths of national $\mathrm{x}$-ray facilities is that they support a broad user program, bringing together researchers from academic institutions, government laboratories, and industry not only nationally but worldwide. Because access is free and solely based on the quality of the proposed research, such 
facilities foster research by small groups that often are not from research universities but undergraduate institutions who bring their students. X-ray facilities thus play a major role in student education.

The historical growth of the synchrotron community through 2007 is illustrated in Figure 5.2. The figure illustrates only the number of on-site users that actively participated in experiments. The total number of scientists involved in synchrotron radiation research is considerably higher. History has shown that the growth of the community generally increases with the offered opportunities, e.g., the availability of experimental stations, and we expect this trend to continue.

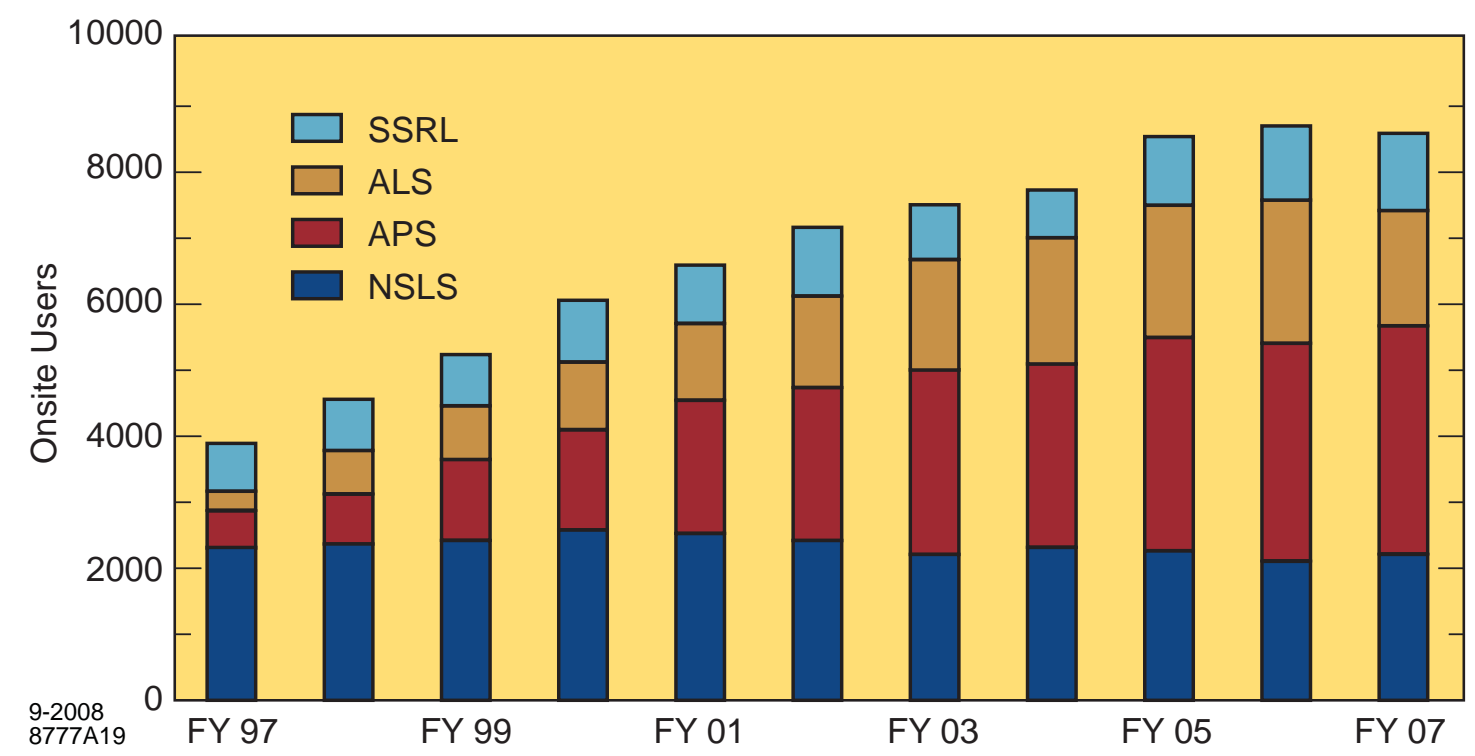

Figure 5.2. Growth of the number of on-site users versus fiscal year of the four BES operated light sources in the U.S. 


\section{APPENDIX A}

\section{Basic Types of X-Ray Facilities}

\section{A.1 Storage Rings}

Storage rings, with electron currents from a few hundred milliamps to the order of $1 \mathrm{amp}$, provide high average flux and brightness (but low peak flux and brightness) x-rays at high pulse repetition rates (up to hundreds of $\mathrm{MHz}$ ). While the inherent electron bunch energy spread is nominally $10^{-3}$ (which degrades the brightness of higher undulator harmonics and long undulators), the pulse-to-pulse energy stability is very high $\left(\sim 10^{-5}\right)$ and meV energy resolution in experiments can be reached. Photon pulse lengths are typically greater than ten picoseconds rms in duration, but shorter pulse lengths can be reached at low current with lattice tuning, and at high current in limited sections of the ring with RF deflection systems and optical manipulations. Laser slicing has been implemented on storage rings to produce $\sim 100 \mathrm{fs} \mathrm{x}$-ray pulses, at repetition rates into the tens of $\mathrm{kHz}$, and with flux per pulse limited by the fractional portion of the bunch charge involved in the process. Storage ring sources provide extremely stable source points and are efficient in operation, have high reliability (>95\% up-time for most facilities), provide tunable output from far IR to hard x-ray (up to tens of $\mathrm{keV}$ in the harmonics of undulators), and support a large user community with several tens of beam lines at each facility.

Horizontal emittance in a storage ring having a given lattice type scales as $E^{2} \cdot \theta^{3}$, where $E$ is the electron energy and $\theta$ is the bend angle for the lattice cell dipoles. Thus, while present facilities reach the diffraction limited emittance for $1-\AA \AA$ radiation (8 pm-rad) in the vertical plane by reducing horizontalvertical coupling and vertical dispersion to very small values, large circumference "ultimate" ring designs (nominally 2-3 $\mathrm{km}$ in circumference) having small $\theta$ and moderate $\mathrm{E}$ are required to approach a diffraction-limited horizontal emittance (in the 10-100 pm-rad range). Such ring implementations offer exceptional average brightness (of order $10^{22}$ photons $/ \mathrm{s} / \mathrm{mm}^{2} / \mathrm{mrad}^{2}$ per $0.1 \%$ bandwidth). Because the ring dipole magnet fields are typically very low in these machines, high power wigglers might be used to provide the radiation damping required to reach low emittance. Ultra-low emittance rings tend to have a small dynamic aperture which makes normal multi-pulse refilling of bunch charge during injection more difficult. An on-axis injection scheme that replaces the full charge of a bunch train in one shot might be needed to realize the very lowest emittance rings. Linac current limitations could restrict the attainable current in the storage ring with this on-axis injection mode unless a separate accumulator ring is used to stack bunch charge prior to injection. The beam lifetime in these machines will be very short due to the small bunch volume. The short lifetime can be improved using bunch-lengthening RF cavities, but will nevertheless necessitate frequent top-off or bunch-replacement injection.

Preliminary studies indicate that when the electron emittance is at or below the diffraction limit for a given wavelength in both planes, partial SASE lasing with the stored beam is possible if the peak bunch current is sufficient with only a small impact on electron emittance and energy spread. Seeded lasing in a storage ring, at the repetition rate of the seed laser, has already been demonstrated in the EUV.

Very low emittance ring performance is limited by intrabeam scattering (which determines the minimum attainable emittance for finite bunch current), fast-ion and possibly electron -cloud instabilities, impedances from the vacuum chamber and coherent synchrotron radiation (CSR) that lead to bunch 
instabilities, synchrotron radiation and RF heating from the relatively high stored beam current, and orbit and beam line component stability. In addition, having an affordable ring size represents a practical limitation.

\section{A.2. Energy Recovery Linacs (ERLs)}

ERLs are a potential source for future generation synchrotron light sources that combine some of the qualities of storage rings with those of linac-based light sources. A CW superconducting RF (SCRF) linac provides high repetition rate beams extending to the RF frequency of the accelerating structure (up to $\mathrm{GHz}$ ) and, for the highest beam power, the ERL configuration is advantageous in providing an affordable RF power system (although the cryogenic system might be quite costly) by recovering the energy of the electron beam. This emerging technology promises to provide very high average brightness with high spatial coherence ( $\sim 50 \%$ or more) by preserving the very low emittance (which scales as $1 / \mathrm{E}$ for linac energy E) and low energy spread achievable from a full-energy, high-current superconducting linac ( 10 pm-rad and $10^{-4}$ respectively), for a single turn around a ring-like transport lattice accommodating several insertion devices. The electron beam would then be returned to the linac where it is decelerated to recover the energy in the superconducting RF structure. Growth in the energy spread of the beam, which occurs as the bunches traverse the ring, must be controlled to avoid beam loss in the linac structure during deceleration.

When operating with the design goal current of $\sim 100 \mathrm{~mA}$, ERL average brightness would exceed $10^{22}$ photons $/ \mathrm{s} / \mathrm{mm}^{2} / \mathrm{mrad}^{2}$ per $0.1 \%$ bandwidth from a long undulator $(\sim 25 \mathrm{~m})$, and the inherently small beam energy spread would enable greater brightness at higher undulator harmonics than can be reached with a storage ring. While the bunch length is on the order of 1 ps in high current ( 100 mA), high brightness mode, a short-bunch, low repetition rate $(\sim 1 \mathrm{MHz})$ mode is envisioned that would provide bunch lengths on the order of tens of femtoseconds at the expense of much lower current, and higher emittance. Dozens of high performance beam lines can operate simultaneously on an ERL; the number is ultimately limited by the growth of beam emittance which should not be more than a few tens of percent. In short-bunch mode, coherent synchrotron radiation in dipoles can increase emittance and energy spread as well, although the mitigation of these effects by longitudinal bunch shaping is being explored. When every bucket is filled, the electron charge per bunch for high-current ERL operation is limited to $<100 \mathrm{pC}$ in today's designs by higher order mode heating in the superconducting linac and by wake-field driven beam break-up. Larger bunch charges need designs with more extensive higher-order-mode absorbers. A total current of up to $\sim 100 \mathrm{~mA}$ might be reached by filling every closely spaced bunch ( $\sim 0.7 \mathrm{~ns}$ spacing for the nominal 1.3-GHz linac frequency) in a large ERL ring with $77 \mathrm{pC}$ per bunch. However, ERLs have flexible fill patterns so that more charge per bunch is possible for reduced bunch repetition rate.

Low-energy ERLs are already being used to drive infrared FELs. Future high-energy ERLs might provide sufficiently small emittance, high peak current and low energy spread to drive x-ray FELs. ERL-driven single-pass SASE and seeded FELs providing very high peak brightnesses have been envisioned and x-ray cavity oscillators (XFELOs) are being investigated (see Section A.3). Recirculation is also appealing as a means of potentially significant cost control, but issues of beam quality preservation require detailed exploration of CSR, including edge radiation, space charge effects, halo, and magnetic field quality during bending and compression. In the case of the exploratory x-ray FEL cavity oscillator, the number of bunches, and thus average current, would have to be drastically reduced (from $100 \mathrm{~mA}$ to $\sim 0.025 \mathrm{~mA}$ ) to limit the cavity power to $20 \mathrm{MW}$ or less. In this case, the XFELO would operate either in a dedicated mode or simultaneously with other beam lines if a separate, higher energy injector for the FEL is used in 
tandem with the normal ERL injector so that the FEL drive beam can be separated from the high current ERL beam after acceleration to high energy.

ERLs having the properties needed for future x-ray light sources face several technical challenges and are presently under development in the US, Japan and Europe. Performance limiting factors include cathode lifetime, the length of the linac and its cooling requirements, which limit beam energy, beam break-up and higher-order-mode heating in the linac structure, space charge limitations to low emittance in the gun for high bunch charge, effects on the electron beam from incoherent and coherent synchrotron radiation, nonlinear beam dynamics, ion trapping, beam-loss control, and orbit instability from ground motion.

\section{A.3 Free Electron Lasers (FELs)}

A free-electron laser is a source of coherent radiation that may be implemented on a linac, storage ring, ERL, or other electron source of suitable beam quality. An FEL introduces micro-bunching of the electron beam, with periodic enhancement of the charge density forming micro-bunches, separated by a distance equal to the wavelength of the emitted radiation. These micro-bunches begin to radiate coherently, significantly increasing the peak brightness. FELs can be either single pass, high-gain type, or an oscillator type making use of high reflectivity mirrors. The single pass, high-gain type can further be operated in two modes, SASE and seeded. In SASE FELs, the microbunching arises from the interaction of the electron beam and the synchrotron radiation emitted upstream in the undulator; seeded FELs introduce an external coherent light pulse that produces microbunching in the electron beam. In an oscillator FEL, a light field develops inside an optical cavity as the undulator radiation from previous passes is trapped and filtered by the mirrors and amplified by interacting with new electron bunches in the undulator. The spectral brightness of an FEL can be billions of times higher than that of a typical storage ring insertion device. In addition, light from an FEL has full transverse coherence and, in some FELs, a very high degree of a temporal coherence. Using the same electron beam in FELs and other coherent synchrotron radiation devices, each at a different wavelength, offers capabilities for synchronized multi-color sources. Future FEL performance may encompass a wide range including the envelopes shown in Section 4, dependent on details of design.

The electron beam quality for an x-ray FEL must exceed that of a storage ring, and this is typically provided by a linac. The electron beam itself must have laser-like quality, with normalized transverse emittances $<1 \mathrm{~mm} \cdot \mathrm{mrad}$, relative energy spread $\sim 10^{-4}$, and peak current of hundreds of amperes to kiloamperes. Limitations arise from the thermal emittance of the electron source, and transverse and longitudinal emittance growth from space-charge, CSR, and impedance effects. Bunches may have up to $1 \mathrm{nC}$ charge and achieve these parameters, although production of ultra-short bunches optimized for lasing with only 1-100 pC charge per bunch has the potential to overcome some limitations to emittance and to produce ultra-bright beams and intense hard x-ray pulses with femtosecond or shorter duration.

Existing FEL facilities are limited to $200 \mathrm{eV}$ photon energy, and the currently achieved normalized emittance of $1 \mathrm{~mm} \cdot \mathrm{mrad}$ at $1 \mathrm{nC}$ charge requires that the beam energy be greater than $10 \mathrm{GeV}$ for lasing at photon energies extending to several keV. Planned SASE FELs will provide short $\mathrm{x}$-ray pulses (tens to hundreds of fs), of unprecedented peak power $(10 \mathrm{GW})$, and peak brightness is expected to be of the order of $10^{33}$ photons $/ \mathrm{s} / \mathrm{mm}^{2} / \mathrm{mrad}^{2}$ per $0.1 \%$ bandwidth, many orders of magnitude greater than storage ring or ERL incoherent sources could provide. Implementation of optical manipulations could allow exquisite control of the electron beam, and development of techniques such as high-gain harmonic generation (HGHG), harmonic cascade, enhanced SASE (ESASE), and self-seeding, are expected to enhance 
performance of planned and future FELs, by controlling x-ray pulse duration ( $<1-100$ s fs), imparting a very high degree of temporal coherence, providing synchronization to another laser for pump-probe experiments, and increasing peak current, thus reducing the length of the radiator undulator required.

Soft x-ray FELs may be seeded by conventional lasers (including HHG sources), by self-seeding wherein monochromatized radiation from an upstream FEL is used to coherently seed the electron bunch which radiates again in a downstream undulator), cascade seeding FELs (a series of self-seeded stages), or in an oscillator configuration in which the photon pulse interacts with the electron beam in an undulator on each pass through a cavity. Seeded hard x-ray FEL types include self-seeded FELs, cascade seeding FELs and X-ray FEL oscillators (XFELOs, or regenerative amplifier FELs-RAFELs). Seeded FELs may be able to operate with high stability at pulse energies below saturation, reducing the peak output flux and brightness in a controlled way. Seeded long ( ps) pulses of close to transform-limit quality could provide meV resolving power, as could FEL oscillators. Seeding by conventional lasers and harmonic generation in an FEL has been demonstrated at VUV wavelengths. Feedback control of the seed laser could shape the amplitude and phase, optimizing x-ray pulse parameters for experiments. For oscillator FELs, high bunch repetition rate is required to minimize the length of the optical cavity, in which the photon pulse interacts with the electron beam in an undulator on each pass through the cavity. Oscillators may be driven with pulse repetition rates on the order of 1-10 MHz, either by ERLs, CW superconducting linacs, bunch trains from a pulsed linac, or in recirculating-linac configuration in which the final beam energy is achieved by multiple passes through a single linac. The wavelength of existing FEL oscillators is currently limited to the VUV range by the availability of optics for the mirrors having high-reflectivity at high incidence angle. Extending oscillator implementations to the hard x-ray region requires Bragg-reflecting crystals. Methods to achieve tunability for these sources, requiring complex mechanical systems to adjust and hold stable the position and angle of cryo-cooled, multiple crystal mirrors using optical interferometric techniques are being investigated, but are a major challenge.

Practical repetition rate for x-ray FELs is currently limited by high-brightness electron gun technology, and the facilities currently under construction are planned to use macropulses at $\sim 10-120 \mathrm{~Hz}$ repetition rate. Pulsed linac repetition rates might eventually approach the order of $1 \mathrm{kHz}$ with developments in pulsed high power RF sources and accelerating structures. In bunch-train mode, pulsed linacs can provide high bunch repetition rates (in principle up to the RF frequency of the linac) over the duration of the linac pulse length (approaching ms for superconducting linacs) to achieve average bunch repetition rates that may reach the order of $10^{5}$ bunches/second. In contrast to the high bunch rates achieved in bursts with pulsed linacs, CW superconducting-linac-based FELs promise to provide high pulse repetition rate with even spacing, from tens of $\mathrm{kHz}$ to $\mathrm{MHz}$ or greater as electron gun technology is developed. CW superconducting-linac-based FELs offer a route to higher average brightness with a time-structured beam that is well suited to many experimental needs, particularly pump-probe. The bunches from the linac can be fanned out to multiple undulators, at the expense of proportionately reduced average brightness for each. The individual FELs can be configured for either SASE, seeded, or oscillator mode of operation dependent on experimental requirements, including use of HGHG, harmonic cascade, self-seeding, or oscillator configuration to obtain high temporal coherence.

SASE x-ray FELs using pulsed linear accelerators of $\sim 14-20 \mathrm{GeV}$ are being built, and the repetition rate is limited to somewhat less than $1 \mathrm{kHz}$, by power dissipation in the accelerating structures and other components, for either normal-conducting or superconducting linacs. Average brightness of $10^{25}$ photons $/ \mathrm{s} / \mathrm{mm}^{2} / \mathrm{mrad}^{2}$ per $0.1 \%$ bandwidth may be achieved by using trains of bunches within the macropulse. SASE FELs exhibit pulse-to-pulse wavelength jitter of $\sim 10^{-3}$ and intensity jitter of $\sim 10 \%$ - 
$20 \%$. Seeding is required to reduce these values to $<10^{-4}$ and $\sim 1 \%$ respectively. A higher energy beam, potentially extending to $50 \mathrm{GeV}$, would both increase the power output (approximately linear with beam energy) and extend the emission into the several tens of $\mathrm{keV}$ range. Reducing the electron beam emittance by about one order of magnitude, made possible by reducing the bunch charge to a few to tens of $\mathrm{pC}$, would also allow the extension of the lasing wavelength to about $0.02 \mathrm{~nm}$, or $60 \mathrm{keV}$, at about $10-15 \mathrm{GeV}$ beam energy, using a shorter period undulator. Use of tapered undulators, designed to maintain the FEL resonance condition as the electron beam loses energy along the FEL, may give a further increase in output power.

The great attractions of CW SCRF for future FEL facilities are the high repetition-rate achievable with even pulse spacing, and the capability of supporting a large number of beamlines each with high average flux and brightness, when switching beam between different FELs. CW operation of the accelerator, with feedback loops to control beam position and energy, offers improved stability over pulsed machines. A high-brightness source operating in CW mode could drive an array of many FELs, with each beamline operating at high repetition rate (from $10 \mathrm{kHz}$ to $\mathrm{MHz}$ and higher) and with even pulse spacing. Higher repetition rates could be supported by the linac, and CW linacs offer the potential for increased average brightness as technology is developed. The $\mathrm{x}$-ray wavelength achievable is limited by electron beam quality, and by linac energy (a cost issue). With a small footprint, 2-3 GeV linac, FELs could operate in the VUV-soft $\mathrm{x}$-ray range, with peak brightness up to $10^{32}$ photons $/ \mathrm{s} / \mathrm{mm}^{2} / \mathrm{mrad}^{2}$ per $0.1 \%$ bandwidth, and average brightness $10^{25}$ photons $/ \mathrm{s} / \mathrm{mm}^{2} / \mathrm{mrad}^{2}$ per $0.1 \%$ bandwidth, to all beamlines simultaneously, dependent on pulse duration and wavelength.

Performance limiting factors for linac-driven FELs include the length of the linac and its cooling requirements, which limit beam energy; space charge limitations to brightness in the gun for high bunch charge; effects on the electron beam phase space, including induced modulations in the 10-100 nm length scale, from space charge and coherent synchrotron radiation; and instability from beam spreader components and ground motion.

\section{A.4 High-Harmonic Generation (HHG)}

Wavelengths in the hard ultraviolet or even the soft x-ray spectral region are attainable in the very high harmonics produced when an intense infrared laser pulse is focused into a gas. High-harmonic generation can be produced with temporal and spatial coherence properties similar to the driving laser field, and under special conditions, with sub-femtosecond pulse duration. Conversion efficiency is relatively low, and typically decreases with increasing harmonic order. HHG can provide a source in a single-investigatorsized laboratory, with output pulses synchronized to the driving laser and produced with the same repetition rate. Such sources have been generated using commercial driving lasers at the several-watt level, with repetition rates ranging from $10 \mathrm{~Hz}$ to $10 \mathrm{kHz}$. The cut-off of the harmonic spectrum extends to shorter wavelength as the drive laser intensity is increased, up to a saturation intensity where harmonic generation decreases. By using a gas species with a higher ionization potential, and a high-power longerwavelength drive laser, together with novel phase-matching techniques in the harmonic-generation medium, the spectral cut-off of HHG may be extended into the hundreds of $\mathrm{eV}$ with conversion efficiency of the order of $10^{-6}$. With the availability of $\mathrm{kW}$ average power lasers, average brightness of $10^{17}$ photons $/ \mathrm{s} / \mathrm{mm}^{2} / \mathrm{mrad}^{2}$ per $0.1 \%$ bandwidth may be achieved. HHG sources will be needed to provide the seeding power for FELs, which then act as amplifiers and additional stages of harmonic generation to generate intense soft and hard $\mathrm{x}$-rays. 


\section{A.5 Advanced Accelerator Concepts}

Advanced accelerator R\&D is pursuing a variety of approaches to produce accelerating gradients orders of magnitude greater than conventional RF systems, enabling ultra-compact high-energy linear accelerators. Laser-driven plasma and dielectric-based acceleration techniques are being developed to provide electron beams to drive FELs and other light sources. In the same vein, electron beam driven dielectric and plasmabased accelerators may provide similar high accelerating gradients and, with an RF-photo-injector, could produce drive beams for FELs useful for synchronized soft to very hard x-rays. Successful development of these technologies would allow for the development of compact, ultra-bright, ultrafast radiation sources covering the entire spectral area from $\mathrm{THz}$ to $\mathrm{x}$-rays to gamma ray beams that are intrinsically synchronized to a laser pulse.

\section{A.6 Other Sources}

Other incoherent, lower brightness x-ray sources include smaller laboratory, or industrial-scale, devices. Laser-electron beam scattering (inverse Compton/Thomson) sources are under development to provide high-average-flux hard x-ray beams using a laser beam colliding with an electron beam from an electron storage ring or linear accelerator. These sources are envisioned for local applications extending from crystallography to diagnostic imaging. Laser-plasma sources producing x-rays by electron acceleration within plasma channels are also under investigation as small laboratory sources. 
 \\ APPENOXB Research and Development Requirements for Sources, Optics, and Detectors}

\section{B.1 X-ray Source R\&D Requirements}

The new x-ray sources that will come on line in the next few years - including the PETRA-III and NSLSII low-emittance storage rings and the LCLS, XFEL, FERMI, SPring-8 XFEL, and SPAR-X FEL facilities - will open a new window into the exploration of matter at the atomic and molecular level. These facilities, however, are only a step along a road of x-ray source and technology development that has the potential to provide even greater breakthrough opportunities for science. Further development of X-ray facilities will provide new levels of performance, thus allowing the acquisition of new knowledge with its associated economic, security, and societal benefits. Future advances in electron sources, normalconducting and superconducting accelerator and other RF structures, advanced manipulations of electron beams, laser systems, insertion devices, x-ray optics and instrumentation will enhance the performance and operational flexibility of soft and hard x-ray sources, while the development of high gradient RF, laser and plasma accelerators will make them more compact, less expensive and more widely available to scientists world-wide. Developments in storage ring accelerator and beam line optics technologies will enable the realization of "ultimate" ring sources having very high brightness, high transverse coherence at angstrom wavelengths and the potential for partial lasing with the stored beam at soft x-ray wavelengths. And, just as atomic and molecular lasers have been developed over the years to reach new levels of power, short pulse length and other characteristics, in the same way short wavelength FELs can be developed to operate at wavelengths shorter than $1 \AA$, and with increased peak power, full transverse and longitudinal coherence, attosecond pulse lengths, and more.

Going from the conceptual level, to the proof of principle, to the implementation of new concepts, requires a strong research program and facilities dedicated to the development of advanced x-ray sources. The principle areas of x-ray source technology that must be developed to establish the technical feasibility of future advanced $\mathrm{x}$-ray sources are discussed in the following.

Electron guns: Continued R\&D in high-brightness electron beam production will benefit future FEL and ERL performance, offering the prospect of higher brightness photon beams with increased coherence and at shorter wavelengths. Developments in high-repetition-rate photocathode, thermionic, and field-emitter electron guns are ongoing and involve normal-conducting and superconducting RF systems, as well as high-voltage DC systems, to produce high-brightness beams at $\mathrm{MHz}$ to $\mathrm{GHz}$ repetition rate and normalized emittances of 0.1-1 mm.mrad at bunch charges of $10 \mathrm{pC}$ to $1 \mathrm{nC}$, and with high reliability and long lifetime. Extremely small normalized emittance $(\sim 0.1 \mathrm{~mm} \cdot \mathrm{mrad})$ may be achieved with low bunch charge ( 1-100 pC), and together with development of bunch compression techniques, this offers a route to highbrightness ultra-fast bunches for FEL and ERL applications.

Superconducting linacs: CW superconducting RF systems need further development for high-current applications in ERLs. New cavity shapes, optimized for higher current, higher bunch charge, low emittance beams, and higher gradient, would have a significant role in improving performance and reducing costs. Cavity HOM damping is required to control multipass beam breakup instabilities. Power 
lost to HOMs from a high-power ERL beam, and particle loss, must both be minimized to limit the load on the cryogenic system. The energy spread of the decelerated beam in an ERL will experience adiabatic antidamping, so the beam energy spread from impedances must be limited to avoid particle losses prior to reaching the beam dump.

High-gradient linacs: High gradient normal conducting RF systems need further development for application to compact x-ray light sources. Sample accelerator structures have been demonstrated with gradients as high as $150 \mathrm{MV} / \mathrm{m}$, roughly 6 times higher than that in the European XFEL. Cavity HOM damping, required for controlling the multibunch beam breakup instabilities, and single-bunch wakefield control techniques both need to be demonstrated. Development of the high peak-power RF systems is also required. Advances in high ultra-high-power RF techniques and high-gradient RF structures hold the promise of developing compact and cost effective x-ray sources. DOE OHEP has historically supported this $R \& D$, but additional targeted $R \& D$ is needed for $x$-ray source applications

Low-emittance beam transport, control, and manipulations: Understanding of the evolution of the electron beam emittance, from cathode to photon source, and means of its control and manipulation, are essential to achieving the highest brightness beams needed for future light sources. Increasingly sophisticated means of electron beam manipulations are on the near horizon, and promise enhanced capabilities and levels of performance for future light sources. Examples include transverse to longitudinal emittance exchange, bunch compression techniques, and beam conditioning for FEL applications. Not all of these techniques can be tested on existing accelerators, so we must develop many of the concepts, and validate the methodologies, with dedicated development accelerator(s) designed for the purpose.

Simulation and validation of accelerator and FEL design: Multi-physics computer modeling tools need to be developed to allow the full start-to-end simulations required for advanced facilities with highbrightness beams. X-ray FEL performance is extremely sensitive to the details of the electron beam phase-space distribution, and design using state-of-the-art simulation tools is required for the next generation of x-ray FELs. Simulations must be validated in experiments on existing and planned accelerators.

Laser systems: Developments in sophisticated laser systems with high average powers will be required for optimal performance of future light sources, benefiting rings, FELs, and ERLs. Applications are in high-brightness photocathode sources, seeding, slicing and optical manipulations, precision timing and synchronization systems, and end-station lasers. Short-pulse lasers in the micron wavelength range, extending average powers up to the $\mathrm{kW}$ level, together with novel phase-matching techniques in the harmonic-generation medium, give promise of future HHG sources extending into the hundreds of eV region with average soft x-ray power in the range of milliwatts. Development of means to control HHG laser system output phase and amplitude is required to enhance their applications in seeding FELs. Such advances in laser technology will have wide-ranging benefit for future light sources operating at high repetition rate.

Seeding and optical manipulation: Seeding by conventional lasers and harmonic generation in an FEL has been demonstrated at VUV wavelengths. However, demonstration of control of electron beams that are seeded at UV and shorter wavelengths, and their performance in harmonic generation at x-ray wavelengths, is needed. Development of control techniques to shape the x-ray pulse via phase and amplitude adjustments to the seed laser may provide significant enhancements to the capabilities of future FELs. Tests of optical manipulation technologies already proposed at a number of FEL facilities are required. Optics for an XFEL oscillator, with excellent stability and performance, also need to be developed. 
RF technology: High power RF transverse and longitudinal deflecting and accelerating structures can be used to create short or long bunches in storage rings (e.g., transverse deflecting "crab" cavities and highharmonic longitudinal accelerating cavities) as well as to implement very fast kickers for on-axis bunch replacement injection to ultra-low emittance storage rings. While development of these components, together with efficient sources of very high RF power to drive them and the RF undulators mentioned below, has been supported in the past by the DOE's high-energy physics program, further targeted R\&D is needed for $\mathrm{x}$-ray source applications.

Laser- and electron-driven acceleration: Continued advanced accelerator $R \& D$ is required for development of high gradient laser-driven and electron beam-driven wakefield technology for light source applications. This technology would enable development of compact, ultra-bright, ultrafast radiation sources covering the entire spectrum from $\mathrm{THz}$ to gamma rays. Intrinsic pump-probe synchronization is achieved with laser-based accelerators and electron driven plasma-wakefield accelerators operating with a photocathode gun. The latter technology offers relatively high efficiency in converting wall plug power to beam power. FEL experiments with laser-plasma systems are now being built, and developments are required in further reduction of the electron beam energy spread and emittance, improvements in accelerator tuning and stability, and increasing the average brightness through use of higher-power laser systems. R\&D to develop large-aperture dielectric structures (to enhance peak brightness), and experimental demonstration of acceleration gains of tens of $\mathrm{MeV}$ are required. Experimental facilities in which to test these and other advanced approaches to particle acceleration and x-ray production are required.

Insertion devices: Development of high performance insertion devices plays a key role in enhancing storage ring, ERL, and FEL brightness and photon energy range. Improvements in in-vacuum and superconducting short-period undulators, and with control of polarization, are needed. As electron beams with smaller emttances and higher brightness are developed we will need undulators with smaller periods, and large gap-to-period ratio. Development of RF undulator technology to further reduce the period while maintaining a sufficient gap for beam transport, and efficient high power drive components, would enable polarization switching at kilohertz rates, and might push the undulator period into the $\mathrm{mm}$ range.

High stability mechanical systems: To reach the daunting stability goals for the extremely small photon beams from future x-ray sources, ultra-high stability mechanical designs for critical accelerator and beam line components will be needed in addition to stabilizing feedback and noise-rejecting detector data acquisition methods. These mechanical components include accelerator magnet support structures, beam position monitor assemblies, beam line mirrors, monochromators and other optical components, and experiment sample and detector assemblies. These components need to be stabilized against temperature variations, ground vibration, cooling water flow, electrical and magnetic influences and other "cultural" disturbances.

Instrumentation, control and timing technology: Paramount to the successful realization of any of the future accelerator and photon beam line technologies mentioned above is the concomitant need to develop the advanced instrumentation, control and femtosecond timing technologies they require. Ultra-low emittance storage ring, FEL, and ERL facilities will require very high precision beam position detectors and integrated stabilizing feedback systems in the accelerator and beam lines. High-current storage rings will require sophisticated control algorithms to manage the large transients in the RF cavity systems and the interplay between accelerating and bunch lengthening RF systems. Advanced wideband RF and beam feedback systems will be needed to control impedance-driven instabilities. Short-pulse FEL facilities require highly stable timing and synchronization systems. High-resolution beam diagnostics will be needed to monitor and control micron-sized electron beams, with picocoulomb current. In some situations, 
close interaction between accelerator and beam line component and detector instrumentation will be required. A continuing R\&D program is needed for these and other instrumentation, control and timing systems.

\section{B.2 X-ray Optics R\&D Requirements}

X-ray optics play an important role in enabling new scientific research at existing and future photon sources and is an integral part of synchrotron facilities worldwide. Based on pioneering work on highquality silicon crystal optics since 1960s and its implementation at early generation of synchrotrons, x-ray optics today encompass a growing portfolio that include both traditional monochromatic and focusing / imaging applications using diffractive, reflective, and refractive x-ray optics. Such growth has been stimulated largely by user needs and superior properties at third-generation synchrotron facilities.

Looking into the future, we envision that several key areas of x-ray optics development are needed in order to take full advantage of existing and future light source facilities as well as new experimental methods. These areas include:

a. high-energy-resolution optics with resolving power of $10^{6}-10^{8}$ for both soft and hard x-rays

b. focusing and imaging $\mathrm{x}$-ray optics to achieve nm-scale spatial resolution

c. ultralow slope-error x-ray mirrors for coherence preservation

d. X-ray optics for generation and utilization of XFELs, including seeding

Associated with the needs in optics development, it will be necessary to integrate stable thermal and mechanical arrangement in the designs of x-ray optics and their environments in order to deliver the desired optical performance.

High-energy-resolution x-ray optics: Inelastic x-ray scattering allows studies of the dynamic behavior of various materials at the molecular. atomic and electronic levels by detecting and analyzing the small energy differences between the incident and the scattered x-rays. For these experiments, high-energyresolution optics are required both for monochromators and for analyzers. Due to difficulties in highresolution $\mathrm{x}$-ray optics and limitations in x-ray flux, at present the best energy resolution available at existing synchrotron facilities is around 1-2 meV. An ultra-high energy resolution of $\sim 0.1 \mathrm{meV}$ is required in order to bring inelastic scattering experiments to the regime of dynamic studies of disordered systems at finite momentum transfers. One promising method to achieve this goal in the hard x-ray domain is to use extreme asymmetric back reflecting Bragg optics that provide an angular dispersion in the diffracted beam, followed by a high-precision angle selector to select the desired x-ray energy within the dispersed band. Similarly in the soft x-ray region, highly dispersive optics based on coherently replicated sliced multilayer gratings look like the best way forward and initial work has shown much promise. Much research and development is needed to achieve this goal and to use such optics in real inelastic x-ray scattering experiments. Further, such optics will necessarily be fixed-energy optics (as determined by the backscattering condition). It will also be necessary to develop high-resolution optics at energies matched to atomic transitions in the solid, for resonance experiments in both the hard and soft x-ray regimes. This too will be a major challenge, but an essential one in order to meet some of the scientific goals outlined in this document.

Nanometer-scale focusing and imaging: X-ray microscopy and imaging has seen a rapid growth at existing synchrotron facilities, with a wide range of scientific applications from materials research and engineering to environmental science to biology. In general, two forms of microscopy and imaging are 
used - scanning x-ray microscopy and full-field x-ray microscopy or imaging. Scanning x-ray microscopy (SXM) makes use of a highly focusing optic to condense an incident x-ray beam down to a small area and raster scan a sample while detecting $\mathrm{x}$-ray transmission, fluorescence, or scattering or combination of all these signals. Full-field transmission x-ray microscopy (TXM) makes use of a high-resolution objective xray lens to magnify a transmitted image through a sample and project it onto an area detector. Both these forms of x-ray microscopy rely on focusing x-ray optics to achieve high spatial resolution. Currently, due to technical difficulties in optics fabrication, the spatial resolution is limited to $15 \mathrm{~nm}$ in the soft $\mathrm{x}$-ray region, and substantially larger in the hard x-ray region. Further developments will rely on substantial additional investment in the new generation of nano-lithography tools now becoming available. In order to satisfy the needs of the scientific community to resolve and identify features of just a few nm in size, the next generation $\mathrm{x}$-ray focusing optics with nm-scale resolving powder need to be developed. Two potential additional ways to achieve this goal are the development of linear Fresnel zone plates based on multilayer deposition, and the development of multilayer profile mirrors with ultralow figure errors. The consensus in the community is that these developments will bring to reality the most powerful x-ray microscopes to allow direct observations of internal structures of matter at nanometer scales, which will revolutionize structural materials science and biology as we know today. The techniques of diffractive imaging, whereby reconstruction of phase from the diffraction pattern of a coherently illuminated object using known constraint information, can lead to significant extension of resolution beyond that now possible today using conventional zone plate based x-ray microscopy. This technique can be used with simple plane wave illumination of an object, or in combination with a SXM microscope in a ptychographic scheme, to enhance the native resolution by a factor of 10 or more. All these methods rely on optics to deliver high coherent power from undulator sources, with as small a wavefront distortion as possible.

Ultra-high quality reflective $\mathrm{x}$-ray optics: X-ray mirrors are used at almost every synchrotron beamline in the world. They serve the purposes of (1) purifying x-ray spectrum by rejecting higher order wavelengths, (2) focusing x-rays to match the specimen dimensions, and (3) removing unused portion of the spectrum for lower power load on downstream optics. As brighter and more coherent x-ray sources become available, the requirements for x-ray mirrors have become increasingly demanding so that they minimize the disturbance to the x-ray beam wavefront. Quality refers to several parameters that are linked together via the Power Spectral Density of height deviations from the perfect optical shape. At the long wavelength end one refers to slope errors, and at the short wavelength end of the PSD to roughness, but the evaluation of the optical performance must span the range of surface frequencies relevant to a particular wavelength of light, and a particular experiment. The requirements for the next generation of ultra-bright sources are significantly beyond our ability to both measure and manufacture at present. Progress in ultra-precision optical and EUV optics has been made in recent years through use of atwavelength interferometric characterization techniques, and correction techniques using ion beam and rheological polishing, and adaption and development of similar technologies to soft and hard x-ray mirrors is showing great promise but requires a substantial further investment. Such precision fabrication, polishing and metrology capabilities does not exist in the United States and substantial investment is required to jump start our effort in order for the US light source facilities to compete on the world scene.

X-ray optics for XFELs: X-ray free electron lasers have stimulated the $\mathrm{X}$-ray community. What is missing is the $\mathrm{X}$-ray analogs of the optics available to the laser community as they build up state of the art experiments. These 'tools' ranging from beam splitters to polarizers to single shot auto-correlators to cross correlators are required to fully utilize the XFELs. Only then will we be able to fully exploit these unique sources. Already today there are concepts and prototypes of crucial elements. An example is the 'split and delay' of x-ray pulses. The developments of these optics will demand wavefront preservation for very thin 
Bragg and Laue beam splitters based on perfect Si crystals or the development of defect free diamond. The focusing elements and monochromators described above will be extremely important.

The SASE sources today provide nearly full transverse coherence but as they are based on the start-up for noise their longitudinal phase space contains spikes in time (energy) that could be eliminated with seeding and using the FEL process as an amplifier and/or a harmonic generation system. These seeding schemes will also require optics development. For example, the monochromators discussed above may will find applications in self seeding schemes,

Of course the ultimate dream is to provide the ability to do coherent control where one can 'shape' the XFEL pulse in phase and amplitude. This has proven to be an exciting research area with 'conventional' lasers and will certainly require novel and demanding x-ray optics to extend into the x-ray area. All in all the availability of the x-ray optics 'toolbox' will transform the XFELs from unique $\mathrm{x}$-ray sources into unique x-ray lasers.

\section{B.3 X-ray Detector R\&D Requirements}

The x-ray photon-based science community is remarkable in that it has essentially neglected the very end of the experiment; the detection and analysis of the scattered $\mathrm{x}$-rays resulting from the various processes described above. The contrast between this community and the high-energy physics (HEP) community is dramatic indeed. The growing complexity of particle physics experiments, coupled with the dramatic growth of the semiconductor electronics industry, transformed nuclear and particle physics from smallscale experiments with off-the shelf commercial electronics into sophisticated $4 \pi$ "laboratories". The need for detector development in the Synchrotron Radiation community has been recognized in two dedicated national workshops (2000 and 2005) and as a key tool that will be needed to answer the Grand Challenges. The need to improve detectors in general is, for example, an integral component of the ESRF upgrade. In addition, all of the new x-ray FEL facilities are actively pursuing detector developments - because they must in order to truly benefit from the new capabilities of their sources. Stunning - often orders of magnitude - advances can be obtained simply by having better detectors. Whether targeting better areal coverage, better spatial resolution, better energy or temporal resolution, significant gains in efficiency are not in principle difficult to achieve, but can lead to dramatically better science..

The key properties for any x-ray detector are:

- spatial resolution and coverage

- energy resolutions

- temporal resolution

- efficiency

- throughput

In addition to these basic functionalities, it will be necessary to provide computational capabilities closely integrated with the basic detector structure, potentially embedded within each sensor element or pixel. Obvious examples are energy histograming for spectroscopy, temporal and spatial cross- and autocorrelations, convolutions and deconvolutions.

\section{Fundamental Limits and R\&D Needed on Detector Materials}

Some of these parameters are limited in principle by the fundamental physics of x-ray detection. The simplest and most widely used detectors are based on the elemental semiconductors germanium and silicon. In either case their energy resolution is limited by carrier productions statistics to around $100 \mathrm{eV}$ 
for x-rays of a few keV. Cryogenic detectors based on millikelvin temperatures, either microcalorimeters or superconducting tunneling junctions, have provided a breakthrough in energy resolution, bringing values of a few eV. This is not trivial technology, but strides are being made and they hold great promise.

Spatial resolution using simple minded pixellated structures is limited by electron-hole diffusion within the sensitive volume. It is possible to use this diffusion to advantage by encouraging lateral diffusion and measuring the charge over several pixels. A center-of-gravity calculation can then provide sub-pixel resolution. Of course, this makes large demands on the noise levels of such detectors. A detector using this principle has not yet been made for $\mathrm{x}$-ray detection, although the idea is commonly used for HEP detectors.

Today (and tomorrow) more efficient $\mathrm{x}$-ray (and electron and ion) detectors will be needed. Hard and soft $\mathrm{x}$-rays present different challenges for detector media: Silicon, the ubiquitous semiconductor, has high $\mathrm{x}$ ray absorption up to about $10 \mathrm{keV}$. This makes it an ideal general-purpose solid-state detector up to those energies - but with a challenge for soft $\mathrm{x}$-rays: thin "entrance windows" to minimize soft $\mathrm{x}$-ray absorption. Solutions exist (e.g. $\delta$-doping) but are still more R\&D topics rather than turnkey solutions. For higher energies, higher $\mathrm{Z}$ materials are needed, such as Germanium, GaAs and other III-V compounds (e.g. GaN).

Although prototype strip and pixel detectors in these materials exist, technologies for building large arrays of small-pixel devices need to be developed, together with modern compact cryosystems to allow them to be readily deployed. Although the III-V and II-VI compound semiconductors have been promoted as potential room-temperature possibilities, they have so far been rather disappointing.

In addition to semiconductor detector materials, work is needed in several other areas, for example faster phosphors and better photocathode materials. As readout times decrease, phosphor decay times need to be reduced (and, to maintain the same performance, light yield must increase). Improved photocathodes will enhance the performance of micro-channel plates, which have a useful role in streak cameras and as fast timing detectors.

\section{R\&D on Electronics and Readout}

Detectors and electronics have always been closely linked, but the proliferation of microelectronics has blurred the distinction. Modern cameras, based on Active Pixel Sensors, are integrated circuits - the electronics is the detector. The most sophisticated pixel detectors today are the Hybrid Pixels developed for the LHC experiments, which in Europe have been successfully spun-off into other areas (e.g. PILATUS and Medipix). To date, monolithic pixels have almost no in-pixel processing, whereas hybrid pixels can have considerable in-pixel processing - this is because the monolithic pixel detector and its electronics share the same (electrical) substrate, whereas the hybrid pixel is a sensor interconnected with a readout chip. New interconnection techniques (3D, SOI) have the promise to combine the advantages of monolithic and hybrid pixel detectors.

Future needs will stress speed - both high frame rates, and high temporal resolution. This is where SR needs vastly exceed HEP needs: in HEP detectors, by design the occupancy (the number of detector elements with useful information per "event") is quite small (although the event rate may be quite high). For SR, the event rate is lower, but the occupancy is $100 \%$. Understanding how to cope with this data rate will require specific attention from the SR community.

Lastly, R\&D on radiation hardness is essential - integrating the detector with its electronics necessarily exposes the electronics to high radiation levels. A significant effort was needed in the HEP community over the past decade to develop affordable radiation tolerant electronics. The damage mechanisms (and doses) are different in the SR community, and the need to understand and mitigate these effects will be 
APPENDIX B

R\&D for Sources, Optics and Detectors

important.

80 WHITE PAPER 
 \\ APPENOIX C \\ Interactions of X-Rays with Matter: Perturbative Limits and Mitigation Strategies}

Today, the use of x-rays for studying the electronic and atomic structure of matter is based on the premise that x-rays provide information on the sample in the "as-prepared" state, which is either the electronic ground state at the temperature of interest or a state that has been prepared by application of an external stimulus like pressure or fields, or even an electronic excitation by a laser. Although x-ray-induced damage, for example of protein crystals, is sometimes a limiting effect, sample perturbations by the x-ray beam itself either are generally too weak to change the structure of the sample during the measurement or are handled within a linear response approximation, an example being the interpretation of angle-resolved photoemission data in terms of a one-electron spectral function.

With the advent of x-ray sources of unprecedented average and peak brightness, new questions arise as to the cross-over from the weak- to the strong-perturbation regime where the electronic, spin, or atomic state of the sample is no longer in the as-prepared state. In this section, we consider the transition from the weak- to the strong-perturbation regime and discuss methods for mitigating unwanted strong perturbations.

While the strong-perturbation regime can be of considerable interest by itself, for example in AMO physics, it is anticipated that, in general, applications of x-rays will continue to require weakly perturbing $\mathrm{x}$-ray beams. In these cases the sample remains close to the as-prepared state during the measurement, which may record the results of a single shot or an average over many pulses.

In the following, we consider effects associated with a strong perturbation of the sample, and we shall refer to modifications from the as-prepared state as "damage.” It is difficult at present to treat the various aspects of "damage" quantitatively. Furthermore, this complicated problem depends on many different parameters, such as source properties, optics, experimental arrangement, type of the sample, and prepared state of the sample.

It is clear from Figure 2.1 that in general we need to distinguish processes both within and between the thermodynamic reservoirs of atoms, electrons, and spins, and we expect the reservoirs to respond on different time scales. We expect metallic samples to behave differently than insulators and soft matter. Owing to efficient electron transport, metals can be "self-healing" and may exhibit a damage limit that is higher than, for example, soft matter and insulators (although this is not true with, for example, optical radiation). In soft matter, we may create a considerable fraction of broken bonds and radicals, and such radiation damage may prevent the determination of the desired properties of the as-prepared sample. In photoemission experiments, space-charge effects will distort the measured signal. In general, the question of "damage" therefore needs to be considered on a case-by-case basis and depends on many parameters, such as the photon energy, the number of photons per pulse, the pulse duration, the repetition rate, the beam area, the interaction cross-section, the electron mean free paths in the sample, and the thermal diffusion coefficient.

In order to develop a better understanding of the various aspects of "damage", we consider below the example of a ferromagnetic metal where all three thermodynamic reservoirs - the lattice, the electrons and 
the spins—-need to be considered. Also, considerable information has been derived from previous opticallaser experiments.

\section{C.1 Case Study: X-Ray Perturbation of a Ferromagnetic Metal}

Under the influence of the x-ray electric field with intensity $\sim \boldsymbol{E}^{2}$, electrons are excited on the timescale of attoseconds into previously unoccupied electronic states. This photoelectric or x-ray absorption process has by far the largest cross-section, as illustrated in Figure C.1 for Fe metal, and triggers various processes that can modify the sample and lead to "damage.” Figure C.1 shows that x-ray absorption becomes significantly enhanced around absorption edges and increases with decreasing photon energy. Thus x-ray “damage” is most severe around soft x-ray absorption edges.

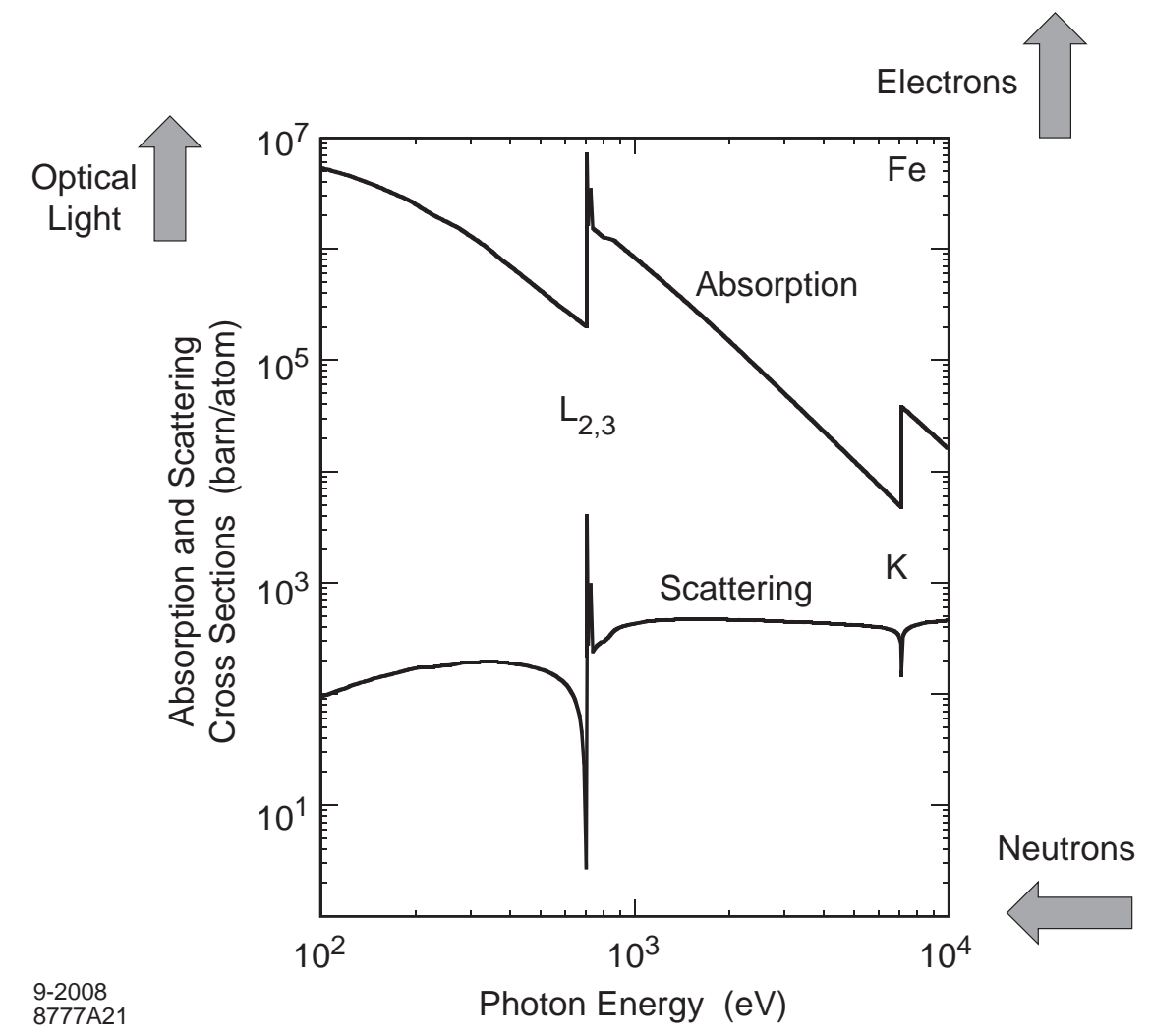

Figure C.1. Energy dependence of the x-ray absorption (photoelectric) and x-ray elastic scattering crosssections for Fe metal in the $100-\mathrm{eV}$ to $10-\mathrm{keV}$ range. Note the resonant enhancements near absorption edges. For comparison, we indicate next to the graph the approximate range of cross-sections for optical photons and electrons, and the better defined cross section for neutrons [Source: J. Stohr]

\section{Response of the Metal on Femtosecond Timescales}

For FEL pulses with lengths of tens of femtoseconds, one needs to consider what happens to the electronic system in the presence of the x-ray pulse, since the signal from the end of the pulse may be modified by changes caused at the beginning of the pulse. This question is of particular interest for soft x-ray experiments that are preferentially sensitive to the electronic instead of the crystallographic structure. Furthermore, x-ray cross-sections are large in the soft x-ray region and electronic perturbations will therefore be most severe. However, other effects may be seen at higher photon energy, where the absorbed energy per absorbed photon is higher. 
In the following, we shall consider an experiment at the Fe L-edge at $\sim 710 \mathrm{eV}$. We neglect linear effects in $\boldsymbol{E}$, such as field distortion and ionization, since they are reduced at x-ray energies because of the high frequency of the fields. We also ignore all effects in the sample arising from the electromagnetic $\boldsymbol{B}$ field since they are negligibly small (cross section smaller by a factor of $<10^{-4}$ of the electric cross-section). Our treatment is restricted to effects following electronic dipole transitions, and within this model we can describe the incoming x-rays by four parameters, the number of photons $N$, the beam cross-section of area $A$, the photon energy $\hbar \omega$, and the pulse length $\tau$. The quantity $N \hbar \omega / A \tau$ thus defines the incident peakpower density.

Nearly all photons get absorbed in the sample over a length $d=2 / \sigma \rho$, corresponding to two photon absorption lengths, where $\sigma$ is the atomic absorption cross-section (plotted in Figure C.1) and $\rho$ the atomic density. Thus the x-ray energy $N \hbar \omega$ contained in $N$ photons is absorbed within a time $\tau$ through electronic excitations in the volume $A d$ containing $\rho A d$ atoms. The deposited energy per atom per pulse is thus given by

$$
U=N \hbar \omega \sigma / 2 A
$$

The electronic excitations occur on individual atoms. For resonant L-edge absorption in Fe at $\sim 710 \mathrm{eV}$, the excitation leads to electrons at energies up to a few eV above the Fermi level and holes in the Fe 2p core shell. Using values for the L-edge excitation of Fe metal, each atom in the absorption volume gets excited (deposition of $\sim 710 \mathrm{eV}$ per atom) for an incident flux of about $3 \times 10^{9}$ photons/pulse $/ \mu \mathrm{m}^{2}$.

The excited electronic states decay on timescales of attoseconds to femtoseconds through Auger decay, radiative decay and electron-electron scattering and, in general, decay and scattering times become shorter with increasing excited state energy. Electrons with kinetic energies larger than the vacuum level $E_{\mathrm{V}}$ may leave the sample as photoelectrons, Auger electrons, and their secondaries. Because the quantum efficiencies of solid samples are less than a few percent and the fluorescence yields in the soft x-ray region are very small, one can safely assume that only a small fraction $(<10 \%)$ of energy leaves the sample. Through repeated inelastic scattering, higher energy electrons produce secondaries, the excitation volume spreads (speeds $>\mathrm{nm} / \mathrm{fs}$ ) beyond the atomic volume, and the secondaries typically end up with a kinetic energy $<E_{\mathrm{V}}$, so that they are trapped by the surface potential barrier (work function). Initially the excited electron gas does not have a well defined temperature which is established only on a timescale of about 100 fs through multiple electron-electron scattering leading to equilibration into a Fermi-Dirac distribution.

The description of the excited electronic state during the pulse is complicated. Optical laser experiments can be used to derive an approximate "damage" threshold on the timescale $>100 \mathrm{fs}$, where the electron gas has equilibrated. In fact, the clearest information comes from the type of experiment illustrated in Figure 3.12c, which has been the subject of extensive work over the last ten years. In such pump-probe experiments on magnetic metals, changes in the electronic state clearly manifest themselves on the $100 \mathrm{fs}$ timescale in changes of the magnetization measured by a delayed weaker probe pulse as a function of the incident power of the pump pulse. For both optical and x-ray excitations, the absorbed photons lead to a "hot" electron distribution extending from the Fermi level to the vacuum level, and one may therefore infer electronic modification thresholds for x-ray experiments from those observed for optical lasers of comparable pulse lengths by comparison of the deposited energy per volume and time.

As shown in Figure 3.12a, it is known that at room temperature the magnetization changes on the time scale of $\sim 100$ fs when a ferromagnet like $\mathrm{Ni}$ is excited with optical photons of 2-eV energy, $<100$-fs pulse 
length and fluences larger than $\sim 1 \mathrm{~mJ} / \mathrm{cm}^{2}$. Since the photon absorption cross-section and absorption length in Fe of $2 \mathrm{eV}$ and $710 \mathrm{eV}$ photons is about the same $(\sim 20 \mathrm{~nm})$, we can take this fluence as our electronic modification threshold, and we obtain for Fe L-edge excitation a threshold of about $10^{5}$ photons/pulse $/ \mu \mathrm{m}^{2}$. This indicates a potential limit in the use of focused beams produced by soft $\mathrm{x}$-ray lasers.

\section{Response of the Metal on Picosecond Timescales}

On longer timescales, the excited electron gas can equilibrate through heat exchange with the phonon bath, and this process will heat up the lattice. While typical electron-phonon scattering times at room temperature are tens of femtoseconds, the energy transfer from the hot electrons to the lattice takes much longer, owing to the large difference in the specific heats of the electron gas and the phonons. The equilibration between the two reservoirs may take picoseconds to tens of picoseconds and may even be bottlenecked. Because of the different electronic and phonon timescales in solids, it is therefore possible to distinguish the effect of the x-rays on the electronic and spin systems from that on the lattice.

A rough estimate of a "damage" limit can be made by simply considering the deposited energy per pulse per atom. In the weak perturbation regime, the deposited energy would have to remain significantly below a typical bond energy of $2 \mathrm{eV} / \mathrm{atom}$. Using the Fe L-edge cross-section $7 \mathrm{Mb}=7 \times 10^{-18} \mathrm{~cm}^{2} / \mathrm{atom}$ at $\hbar \omega=710 \mathrm{eV}$, a deposited energy per pulse of $1 \%$ of this bond energy corresponds to about $10^{5}$ photons/pulse/ $\mu \mathrm{m}^{2}$. This number has the same order of magnitude as that derived earlier for electronic modification.

One may calculate the increase in lattice temperature using the Dulong-Petit value $3 k_{\mathrm{B}}$ of the specific heat capacity per atom. In the absence of heat conduction, the increase in lattice temperature $\Delta T$ is then related to the number of photons per pulse $N$ according to

$$
\Delta T \simeq \frac{N \hbar \omega \sigma}{6 A k_{\mathrm{B}}}
$$

For the same parameters used above, the number of incident photons would therefore need to be limited to about $10^{3} \Delta T$ photons/pulse $/ \mathrm{K} / \mu \mathrm{m}^{2}$, where $\Delta T$ is the tolerable temperature increase. Thus an allowed temperature increase of $100 \mathrm{~K}$ would again yield a limiting value of $10^{5}$ photons/pulse $/ \mu \mathrm{m}^{2}$. Note that this number corresponds to a single pulse and does not take into consideration the repetition rate. On the other hand it assumes no heat conduction out of the volume heated by the x-ray beam. In practice, heat conduction partially compensates for repetition rate effects. The effect of heat conduction is discussed more in Section C.3 below.

These considerations are consistent with the results of early experiments carried out with the FLASH soft $\mathrm{x}$-ray/VUV FEL at considerably higher photon fluences. When a solid sample is subjected to a single unattenuated, nominally 10 -fs-long, FEL pulse, a scattering pattern is obtained from the sample, yet after the pulse, the sample is destroyed by heating the surface to plasma temperatures. Although the spatial resolution of present experiments is limited by the available long wavelength the observations are consistent with the notion that modification of the atomic structure of the sample happens on time scales longer than $10 \mathrm{fs}$. 


\section{C.2 Space-Charge Effects: Photoemission}

The above estimates do not take into consideration the detection of the signal. In photon-in/electron-out experiments, space-charge effects determine the maximum fluence. Such effects become increasingly important when the required energy resolution of the emitted electrons increases.

The first core-level photoemission experiments at FLASH at photon energies of $\sim 120 \mathrm{eV}$, pulse lengths $30 \mathrm{fs}$, and incident photon energy densities up to $5 \times 10^{10} \mathrm{~W} / \mathrm{cm}^{2}$ have revealed such effects. Significant broadening and shifting of the $\mathrm{W} 4 \mathrm{f}$ photoemission lines was observed to set in around $3 \times 10^{7}$ photons/pulse for a photon spot $\sim 300 \times 300 \mu \mathrm{m}^{2}$.

In angle-resolved photoemission experiments on correlated and superconducting materials where very high energy resolution is required, the space-charge effects are even stronger and restrict measurements to considerably lower fluxes. Space-charge effects stem from the Coulomb interaction between electrons created within a pulse (for a low-repetition-rate source) or between secondary electrons from adjacent pulses (for a high-repetition-rate source). In general, a high-repetition-rate source has the advantage over a low-repetition-rate source, since it allows a reduction in the number of photons per pulse while maintaining the same average flux.

\section{C.3 Possible Remedies for Damage}

As occurs in protein crystallography and cryo-TEM, we expect that in some cases damage issues may be reduced by special effects or overcome by experimental procedures. In some cases, analysis techniques may be developed to correct for them. Damage may be reduced by simply changing the photon energy, as evident from the strongly changing absorption cross-section in Figure C.1. For example, in resonant coherent imaging (speckle) studies one may tune the photon energy to just below the absorption edge where the absorptive (imaginary part) of the scattering cross-section is minimized but the dispersive (real part) is resonantly enhanced. Such "phase images” offer comparable contrast to "absorption images.”

Other experimental remedies consist of spreading the beam over a larger area, e.g., by using a grazingincidence geometry on the target. Brightness-hungry experiments are often performed under conditions where the beamline optics filters out much of the flux, as when one requires a very high-resolution monochromator or when zone-plate focusing optics are used. In these cases the source-produced intensity is naturally reduced. Other examples involve tenuous samples where absorption is low, as with lowpressure gases and aerosols. In some cases, one may overcome the damage problem by continuously refreshing the sample using micro- or nano-fluidics, jets, droplets, or by scanning a solid sample or surface.

We can envision two other novel effects, as yet unproven, that may provide a way out of "damage". One is operative on ultrashort time scales, the other for nanosized beams.

As discussed in Section C.1, the electronic state of a sample is ill defined on ultrashort excitation time scales (e.g. less than 100 fs for a metal). One could therefore envision that on such ultrashort timescales, i.e. for pulses that are of order of $1 \mathrm{fs}$, the electronic structure remains frozen in the "as prepared" state during the measurement. Since a new (modified = "damaged") electronic structure becomes uniquely defined only after an electronic equilibration time of about $100 \mathrm{fs}$, one may be able to avoid "electronic damage” with ultrafast pulses. Only at a delay time of a few hundred femtoseconds would a second pulse 
then see the modified electronic state and at delay times of picoseconds it would probe the atomically excited sample, which may even have been destroyed.

Another effect may prevent physical "damage" of a sample when the illuminated sample volume becomes very small, e.g. for beams with lateral sizes smaller than a micrometer. In our earlier discussion of "damage", we have considered only the energy deposited in the sample volume illuminated by the beam but not the heat flow out of the volume. In general, this assumption overestimates "damage" effects. Furthermore, we have used the simple metric of constant fluence or energy per unit area to determine damage limits. As the area decreases, this metric leads to a drastic reduction in the tolerable number of incident photons per pulse. In practice, in this way we may vastly overestimate damage effects. We know that electron transport in macroscopic structures like wires is limited to certain current densities because of heating and the ultimate melting of the wire. If the macroscopic rules were applied to nanowires, much of our present technology would not be possible. For example, a macroscopic $\mathrm{Cu}$ wire of 1-mm diameter has a current density survival limit of about $10^{3} \mathrm{~A} / \mathrm{cm}^{2}$. In metallic Cu nano-pillars of $\sim 100$-nm diameter one can achieve current densities $10^{8} \mathrm{~A} / \mathrm{cm}^{2}$ without destroying the device. This increase of five orders of magnitude is due to the significantly increased surface-to-volume ratio and the resulting high cooling rate through the surface of the nano-region. We expect similar "cooling" effects when the x-ray beam size is reduced, and hence the physical damage limit would be significantly increased. We note, however, that such thermal equilibration proceeds on the slower pico- to nanosecond time scales. 


\section{ACKNOWLEDGEMENTS}

This document was coordinated by, and includes written contributions from, the team listed at the front of the document.

We acknowledge written contributions from:

John Arthur (SLAC)

Hendrik Bluhm (LBNL)

Axel Brunger (Stanford)

Larry Carr (BNL)

Andrea Cavalleri (Univ. of Hamburg, Germany)

Eric Colby (SLAC)

Tom Devereaux (Stanford/SLAC)

Kelly Gaffney (Stanford/SLAC)

Oliver Gessner (LBNL)

Janos Hajdu (Uppsala)

Britt Hedman (Stanford/SLAC)

Russel Hemley (Geophysical Laboratory)

Alexander Hexemer (LBNL)

Keith Hodgson (Stanford/SLAC)

Georg Hoffstaetter (Cornell)

Raymond Jeanloz (Berkeley)

Kwang-Je Kim (ANL)

Richard Lee (LLNL)

Wim Leemans (LBNL)

Aaron Lindenberg (Stanford/SLAC)

Wendy Mao (Stanford/SLAC)

James Misewich (BNL)

James Murphy (BNL)

Anders Nilsson (Stanford/SLAC)

Claudio Pellegrini (UCLA)

James Safranek (SLAC)

Peter Siddons (BNL)

Hans Siegmann (SLAC)

Christoph Steier (LBNL)

Hiro Tsuruta (SLAC)

Vittal Yachandra (LBNL)

Bill Weiss (Stanford/SLAC)
We acknowledge helpful comments from:

Paul Adams (LBNL)

Michael Borland (ANL)

Joe Bisognano (SRC)

Phil Bucksbaum (SLAC)

Robert Byer (Stanford)

Yunhai Cai (SLAC)

Swapan Chattopadhyay (Cockroft Institute, UK)

Jeff Corbett (SLAC)

Winfried Decking (DESY, Germany)

Peter Denes (LBNL)

Seb Doniach (Stanford)

Pascal Elleaume (ESRF, France)

William Fawley (LBNL)

Ben Feinberg (LBNL)

John Fox (SLAC)

Miguel Furman (LBNL)

Efim Gluskin (ANL)

Rod Gerig (ANL)

M Zahid Hasan (Princeton)

Toshio Kasuga (KEK, Japan)

Steve Kevan (Univ. Oregon)

Sam Krinsky (BNL)

Steve Leone (Berkeley/LBNL)

Donghui Lu (SLAC)

David Moncton (MIT)

Howard Padmore (LBNL)

Hirohito Ogasawara (SLAC)

Dave Robin (LBNL)

Eli Rotenberg (LBNL)

Ronald Ruth (SLAC)

Andy Sessler (LBNL)

Sami Tantawi (SLAC)

Hans Weise (DESY)

Herman Winick (SLAC)

Mike Zisman (LBNL) 